\title{
Interval Type-2 Fuzzy Multiattribute Group Decision-Making for Logistics Services Providers Selection by Combining QFD with Partitioned Heronian Mean Operator
}

\author{
Hui Zhang, ${ }^{1}$ Hui Gao $\left.\mathbb{D}\right)^{1,2}$ and Peide Liu $\mathbb{D}^{2}$ \\ ${ }^{1}$ School of Business, Heze University, Heze, Shandong, China \\ ${ }^{2}$ School of Management Science and Engineering, Shandong University of Finance and Economics, Jinan 250014, Shandong, China \\ Correspondence should be addressed to Hui Gao; gaohui@mail.sdufe.edu.cn
}

Received 7 December 2018; Revised 6 March 2019; Accepted 21 March 2019; Published 2 May 2019

Academic Editor: Mojtaba Ahmadieh Khanesar

Copyright (c) 2019 Hui Zhang et al. This is an open access article distributed under the Creative Commons Attribution License, which permits unrestricted use, distribution, and reproduction in any medium, provided the original work is properly cited.

Logistics service (LS) has key impacts on customers' satisfaction. Quality function deployment (QFD) can guarantee that logistic services provider's (LSP) attributes are in accordance with the customer's preferences for the LS. The partitioned Heronian mean (PHM) operator assumes that all attributes are partitioned into several clusters. Herein, the attributes in the same clusters are interrelated, while the attributes in different clusters are independent, and the operator can be utilized to solve LSP selection (LSPS) problems in which all attributes are partitioned into several clusters. Interval type- 2 fuzzy sets (IT2FSs) can more competently express the ambiguity and vagueness and have more powerful processing abilities. In this paper, we propose a novel LSPS method from the customers' perspective by combining QFD with the PHM operator in IT2FSs environments. First, we develop the interval type-2 fuzzy PHM (IT2FPHM) operator and the interval type-2 fuzzy weighted PHM (IT2FWPHM) operator and discuss some properties of them. Then, based on the relationships between the customer requirements (CRs) and the technical attributes (TAs), QFD is utilized in order to convert the CRs for LS concerns into multiple attributes for LSP's TAs. Finally, a case of a fresh E-business LSPS is used in order to demonstrate the validity and rationality of the proposed method, and some comparisons are used in order to show the superiority of the proposed method.

\section{Introduction}

As global competition becomes increasingly keen; many businesses are trying to decrease costs, increase efficiency, and improve service by means of logistics $[1,2]$. The demand for the number and type of logistics services providers (LSP) is increasing [2]. In addition, LSP selection (LSPS) has become a decision for businesses that attempt to highlight their core competence and entrust their other noncore activities to some technical and specialized companies [3]. Therefore, selecting the most suitable LSP is a significant and critical decision that is complex because of the existence of various ambiguity and uncertainly based attributes. $\mathrm{Hu}$ et al. [4] developed a multiobjective mixed-integer nonlinear programming model to determine the optimal selection strategy of function LSP. Martikainen et al. [5] developed a process of building potential business models for an LSP operating in a local food supply chain. Chen et al. [6] introduced an evaluation system of LSP for omnichannel environment by identifying the probability of the logistics provider fulfilling a criterion.

In this regard, multiattributes decision-making (MADM) or multiattributes group decision-making (MAGDM) techniques can make it easy for businesses to solve this decision problem [7]. This is because the LSP can be evaluated based on the conflicting attributes. The MADM or MAGDM method can choose the most suitable LSP among the candidate LSPs. Li et al. [8] presented a hybrid information MADM method based on integrated cumulative prospect theory to solve LSP problems. Pamucar et al. [9] proposed a novel integrated interval rough number MADM method based on the multiattribute border approximation area comparison. Wang et al. [10] introduced a hybrid MADM method by combining Decision-Making Trial and Evaluation 
Laboratory (DEMATEL), analytic network process (ANP), and fuzzy analytic hierarchy process (FAHP) to deal with reverse logistics enterprises selection problems.

However, in the real LSPS process, due to indeterminacy and inaccuracy, the evaluated values (EVs) of most of attributes, such as green competencies, pollution control, social responsibility, respect for freshness, reliability, empathy, quality stability, flexible time of receipt, and so on, cannot be adequately stated using type-1 fuzzy sets (T1FSs). Under the circumstances, type-2 fuzzy sets (T2FSs) can be utilized as a suitable technique for dealing with these indeterminacies and inaccuracies.

T2FSs, which were first developed by Zadeh [11], are known as an extension of T1FSs. The transparent diversity between them is that the memberships of T1FSs are crisp values but the memberships of T2FSs are T1FSs; therefore, T2FSs can more sufficiently reflect ambiguity and vagueness than T1FSs, and the research on T2FSs has attracted increasingly more attention. Considering the computational complexity, interval T2FSs (IT2FSs) [12] are the most vigorously applied T2FSs, and have been extended widely to control [13], identification and prediction [14], order allocation [15], data clustering [16], transportation mode selection [17], global supplier selection [18], etc.

The interval type-2 fuzzy aggregation operators can aggregate some IT2FSs into an overall one, and they are commonly utilized to deal with MADM or MAGDM problems in the context of IT2FSs. Zhang [19] presented the MAGDM approach based on some trapezoidal interval type-2 fuzzy (TIT2F) aggregation operators, such as the TIT2F weighted average (TIT2FWA) operator, the generalized TIT2FWA (GTIT2FWA) operator, the TIT2F order weighted average (TIT2FOWA) operator, the generalized TIT2FOWA (GTIT2FOWA) operator, the TIT2F hybrid averaging (TIT2FHA) operator, and the generalized TIT2FHA (GTIT2FHA) operator. However, these aggregation operators suppose that all the IT2FSs are independent. Obviously, this is unreasonable in the real MADM or MAGDM problems. In innumerable situations, we should consider the objective interrelationships between attributes, and then Heronian mean (HM) operator can complete this function. The HM operator [20], which has some advantages over the Bonferroni mean operator [21], considers the correlation between an attribute and itself and can avoid redundant calculations. Wang et al. [22] introduced a novel MAGDM method based on the TIT2F generalized HM (TIT2FGHM) operator and weighted TIT2FGHM (WTIT2FGHM) operator to deal with provider selection problems.

Nevertheless, in many real MADM or MCGDM problems, the interrelationships between attributes may not always exist [23-26]. For instance, when solving fresh Ebusiness LSPS problems, we take into account these attributes as follows: distribution speed $\left(\mathrm{T}_{1}\right)$, quality stability $\left(\mathrm{T}_{2}\right)$, encasement $\left(\mathrm{T}_{3}\right)$, professional cold storage and transport equipment $\left(\mathrm{T}_{4}\right)$, flexible time of receipt $\left(\mathrm{T}_{5}\right)$, service-oriented attitude $\left(\mathrm{T}_{6}\right)$, and feedback speed $\left(\mathrm{T}_{7}\right)$. Obviously, the higher the technical level of professional cold storage and transport equipment, the stronger the quality stability. Similarly, the greater the service consciousness of a service-oriented business, the faster the feedback speed. Therefore, based on the interrelationships between different attributes, we can divide these seven attributes into the following three partitions: $\mathrm{P}_{1}=\left\{\mathrm{T}_{1}, \mathrm{~T}_{5}\right\}, \mathrm{P}_{2}=\left\{\mathrm{T}_{2}, \mathrm{~T}_{3}, \mathrm{~T}_{4}\right\}$, and $\mathrm{P}_{3}=\left\{\mathrm{T}_{6}, \mathrm{~T}_{7}\right\}$, where $\mathrm{T}_{1}$ is related with $\mathrm{T}_{5}$ and $\mathrm{T}_{1}$ is not related with $\mathrm{T}_{2}, \mathrm{~T}_{3}, \mathrm{~T}_{4}, \mathrm{~T}_{6}$, and $\mathrm{T}_{7}$. With this being the case, the aggregation results are likely to be unreasonable when applying the HM operator. Then, some researchers developed the partitioned HM (PHM) operator, which can break away from the above limitations. Liu et al. [27] presented two MAGDM methods based on the linguistic intuitionistic fuzzy partitioned geometric HM (LIFPGHM) operator and the weighted LIFPGHM operator. However, the literatures on the MADM or MAGDM method based on the PHM operator with IT2FSs information are very few.

In addition, as market competition intensifies, enterprises should conform their managements and operations to meet the customers' requirements and survive in the market competitive environments. Quality function deployment (QFD) is a very powerful tool when the LSPS needs to ensure customers' satisfaction [28]. As a general rule, the QFD transformations are represented by a framework matrix, which is known as the house of quality (HOQ), and customer needs (CRs) can be translated into technical attributes (TAs) according to the relations between the CRs and TAs. In the last few years, the QFD has attracted increasingly more attention, especially for the LSPS. Liao and Kao [29] developed an approach by integrating QFD, the fuzzy extended analytic hierarchy process and multiple segment goal programming to improve logistics services. Yazadani et al. [30] integrated QFD with gray relation analysis in order to assess the importance of the LS indicators in the supply chain. Li et al. [31] proposed a technique using QFD to construct a benchmarked recovery process for the third party reverse LSP.

The LSPS process is analyzed as a combination of CRs and TAs (LSP attributes). That is, the relationship between a customer's satisfaction evaluation attributes (CRs) and LSP attributes (TAs) can be expressed using HOQ. These two kinds of attributes are taken from the customer's perspective and the expert's perspective, respectively. Customers provide EVs about the importance of the customer's satisfaction evaluation attributes and the rating of alternatives. Experts provide EVs about the relation between the customer's satisfaction evaluation attributes and the LSP attributes. After aggregation, the customer's preferences are converted into the rating of the weights of the LSP attributes and the rating of the alternatives regarding the LSP attributes through the QFD [32].

From the literature survey, it can be seen that researchers have already applied many approaches and all kinds of combined approaches to choose the most suitable LSP. However, no attempt from customers' perspective has been made so far to develop a sound mathematical model for dealing with LSPS problems that take into account the interaction between LSP attributes. Furthermore, it also supposes all LSP attributes are partitioned into several clusters where the attributes in the same clusters are interrelated while the attributes in different clusters are independent. That is, there are no studies that address with LSPS problems using the PHM operator 
and QFD, especially, with respect to the information of IT2FSs. Therefore, it is meaningful and valuable to develop a LSPS method based on the PHM operator and QFD in environments with IT2FSs.

Based on the above analysis, the main contributions of this paper are summarized as follows.

(1) We convert the EVs of the importance degrees of CRs into the ones of TAs using the relationships between CRs and TAs based on QFD. Here, the customers provide the EVs of the importance degree of CRs, and the experts assess the relationship between CRs and TAs.

(2) We develop the IT2FPHM operator and IT2FWPHM operator and discuss some properties of them.

(3) We propose an LSPS method based on QFD and the PHM operator using IT2FSs. It can assure that LSP attributes are in accordance with the customer's satisfaction evaluation attributes and can ensure customers' satisfaction by integrating the classical QFD.

(4) We verify the validity and rationality of the presented method using a case study about the fresh E-business LSPS and its superiority is illustrated using some comparisons.

The rest of this paper is organized as follows. Section 2 reviews the classical QFD and some basic concepts and operational laws of IT2FSs. Section 3 develops the novel LSPS method under the IT2FSs based on QFD and the PHM operator. Section 4 addresses the case of a fresh E-business LSPS. Section 5 gives some conclusions.

\section{Preliminaries}

2.1. The QFD Method. QFD [33] was initially developed by Yoji Akao in Japan in 1972. It is a desirable strategic design tool to support enterprises in improving their products and services that takes customers' satisfaction as the directional and significant driving factors in product or service design. The main favorable characteristic of this tool is the ability to select the preferred technical designs that meet customers' demands by bridging the gap between the customers and the product or service design teams. However, in reality, it is hard to evaluate technical designs' overall performance values using CRs, but they can be obtained using TAs. Therefore, converting CR (such as product, service, and so on) assessments into TA (such as product, production, process, management design, and so on) assessments is the crucial work of this tool.

As a rule, the QFD transformations are represented using a framework matrix, which is known as the house of quality (HOQ). The HOQ is the fundamental planning technique of QFD and contains the following six items, as displayed in Figure 1: the CR matrix, the TA matrix, the relationship matrix between CRs and TAs, the relative importance of CRs, the inner interrelationships between TAs, and the relative importance of TAs.

The detailed QFD method contains the following steps.

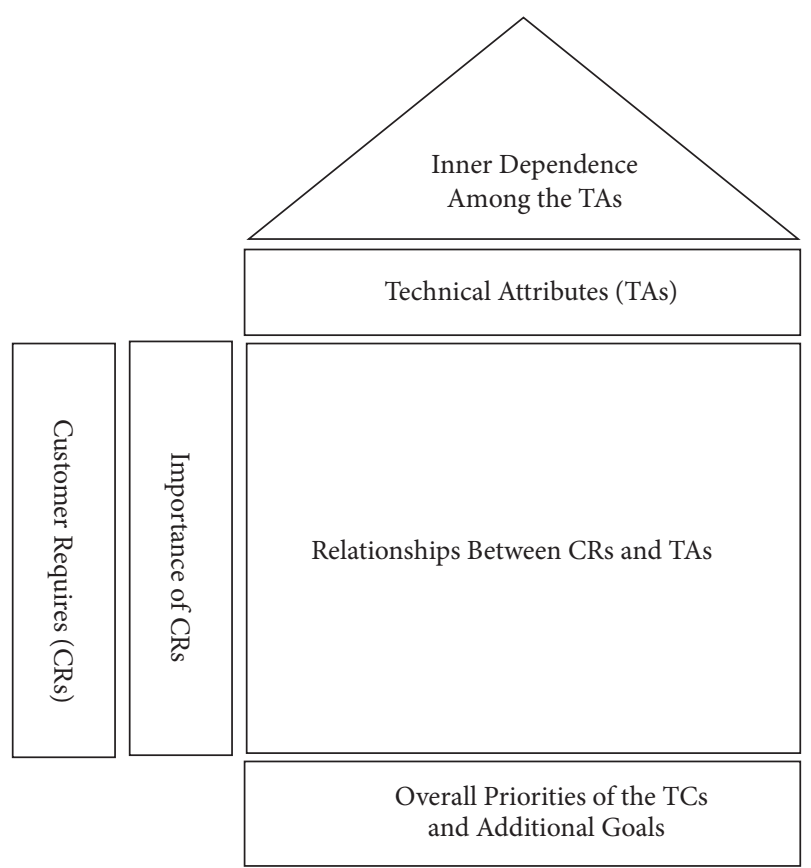

Figure 1: Flow chart of QFD [38].

Step 1 (identify the CRs). The CRs for a specified product or service are first identified. Then, the attributes of the CRs are defined.

Step 2 (determine the weights of the CRs). The customers provide their EVs for each attribute of CRs by applying crisp numbers or fuzzy numbers. The global EVs of the attributes of CRs are obtained using some average operators (such as the weighted average (WA) operator, the power average (PA) operator, and so on).

Step 3 (identify the TAs). The technical attributes are identified based on the attributes of CRs.

Step 4. Determine the relationships between the CRs and TAs and build the corresponding relationship matrix, which states to what degree the attributes of TAs influence the attributes of CRs.

Step 5 (determine the interdependencies among TAs). The correlations between TAs play an important role in QFD applications and are essential in product or service design. Since the PHM operator can effectively deal with these correlations between TAs, in this step, it is unnecessary for us to take them into account.

Step 6. Calculate the weights of TAs based on the weights of CRs and the relationships between the CRs and TAs.

\subsection{IT2FS}

Definition 1 ([34]). Let $\Phi$ be the universe of discourse. A T2FS B can be expressed using a type- 2 membership function $\mu(\phi, \varphi)$ as follows:

$$
\mathrm{B}=\left\{\left((\phi, \varphi), \mu_{\mathrm{B}}(\phi, \varphi)\right) \mid \forall \phi \in \Phi, \forall \varphi \in J_{\phi} \subseteq[0,1]\right\}
$$




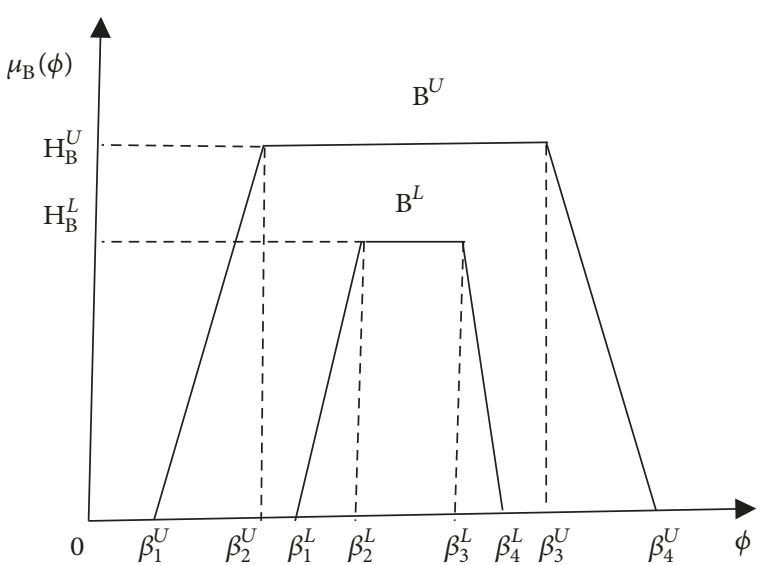

FIGURE 2: A geometrical interpretation of an IT2FS.

where $J_{\phi}$ represents an interval in $[0,1]$. Meanwhile, the T2FS $\mathrm{H}$ can also be expressed as follows:

$$
\mathrm{B}=\int_{\phi \in \Phi} \int_{\varphi \in J_{\phi}} \frac{\mu_{\mathrm{B}}(\phi, \varphi)}{(\phi, \varphi)}=\int_{\phi \in \Phi} \frac{\left(\int_{\varphi \in J_{\phi}} \mu_{\mathrm{B}}(\phi, \varphi) / \varphi\right)}{\phi}
$$

where $J_{\phi} \subseteq[0,1]$ is the primary membership (PM) at $\phi$ and $\int_{\varphi \in J_{\phi}} \mu_{\mathrm{B}}(\phi, \varphi) / \varphi$ denotes the second membership (SM) at $\phi$. For discrete spaces, $\int$ is replaced by $\sum$.

Definition 2 ([34]). Let B be a T2FS in $\Phi$. If all $\mu_{\mathrm{H}}(\phi, \varphi)=1$, then $\mathrm{H}$ is called an IT2FS. This is expressed as follows:

$$
\mathrm{B}=\int_{\phi \in \Phi} \int_{\varphi \in J_{\phi}} \frac{1}{(\phi, \varphi)}=\int_{\phi \in \Phi} \frac{\left(\int_{\varphi \in J_{\phi}} 1 / \varphi\right)}{\phi}
$$

Clearly, IT2FS B in $\Phi$ is totally determined by the PM, which is known as the footprint of uncertainty (FOU). The FOU may be expressed as follows:

$$
F O U(\mathrm{~B})=\bigcup_{\phi \in \Phi} J_{\phi}=\bigcup_{\phi \in \Phi}\left\{(\phi, \varphi) \mid \varphi \in J_{\phi} \subseteq[0,1]\right\}
$$

Because the operations on IT2FSs are complicated, IT2FSs are universally handled by certain simplified forms. In this paper, we apply the trapezoid IT2FS (TIT2FS) to solve MCGDM problems.

Definition 3 ([35]). Let $\mathrm{B}^{L}$ and $\mathrm{B}^{U}$ be two generalized trapezoidal fuzzy numbers where the height of a generalized fuzzy number is positioned in $[0,1]$. Let $h_{\mathrm{B}}^{L}$ and $h_{\mathrm{B}}^{U}$ be the heights of $\mathrm{B}^{L}$ and $\mathrm{B}^{U}$, respectively. An IT2FS B (as shown in Figure 2) in the universe of discourse $\Phi$ can be defined as follows:

$$
\begin{aligned}
\mathrm{B} & =\left(\mathrm{B}^{L}, \mathrm{~B}^{U}\right) \\
& =\left[\left(\beta_{1}^{L}, \beta_{2}^{L}, \beta_{3}^{L}, \beta_{4}^{L} ; \mathrm{H}_{\mathrm{B}}^{L}\right),\left(\beta_{1}^{U}, \beta_{2}^{U}, \beta_{3}^{U}, \beta_{4}^{U} ; \mathrm{H}_{\mathrm{B}}^{U}\right)\right]
\end{aligned}
$$

where $\mathrm{B}^{L}$ and $\mathrm{B}^{U}$ are the T1FSs, $\beta_{1}^{L} \leq \beta_{2}^{L} \leq \beta_{3}^{L} \leq \beta_{4}^{L}, \beta_{1}^{U} \leq$ $\beta_{2}^{U} \leq \beta_{3}^{U} \leq \beta_{4}^{U}, \beta_{1}^{U} \leq \beta_{1}^{L}, \beta_{4}^{L} \leq \beta_{4}^{U}$, and $0 \leq \mathrm{H}_{\mathrm{B}}^{L} \leq \mathrm{H}_{\mathrm{B}}^{U} \leq 1$. The lower membership function (MF) $\mathrm{B}^{L}(\phi)$ and the upper $\operatorname{MFB}^{U}(\phi)$ are defined as follows:

$$
\mathrm{B}^{L}(\phi)= \begin{cases}\mathrm{H}_{\mathrm{A}}^{L} \frac{\left(\phi-\beta_{1}^{L}\right)}{\beta_{2}^{L}-\beta_{1}^{L}} & \beta_{1}^{L} \leq \phi \leq \beta_{2}^{L} \\ \mathrm{H}_{\mathrm{A}}^{L} & \beta_{2}^{L} \leq \phi \leq \beta_{3}^{L} \\ \mathrm{H}_{\mathrm{A}}^{L} \frac{\left(\beta_{4}^{L}-\phi\right)}{\beta_{4}^{L}-\beta_{3}^{L}} & \beta_{1}^{L} \leq \phi \leq \beta_{2}^{L} \\ 0 & \text { otherwise }\end{cases}
$$

$$
\mathrm{B}^{U}(\phi)= \begin{cases}\mathrm{H}_{\mathrm{A}}^{U} \frac{\left(\phi-\beta_{1}^{L}\right)}{\beta_{2}^{U}-\beta_{1}^{U}} & \beta_{1}^{U} \leq \phi \leq \beta_{2}^{U} \\ \mathrm{H}_{\mathrm{A}}^{U} & \beta_{2}^{U} \leq \phi \leq \beta_{3}^{U} \\ \mathrm{H}_{\mathrm{A}}^{U} \frac{\left(\beta_{4}^{U}-\phi\right)}{\beta_{4}^{U}-\beta_{3}^{U}} & \beta_{1}^{U} \leq \phi \leq \beta_{2}^{U} \\ 0 & \text { otherwise }\end{cases}
$$

Definition 4 ([36]). Let $\mathrm{B}_{1}=\left[\left(\beta_{1}^{L}, \beta_{2}^{L}, \beta_{3}^{L}, \beta_{4}^{L} ; \mathrm{H}_{\mathrm{B}_{1}}^{L}\right)\right.$, $\left.\left(\beta_{1}^{U}, \beta_{2}^{U}, \beta_{3}^{U}, \beta_{4}^{U} ; \mathrm{H}_{\mathrm{B}_{1}}^{U}\right)\right]$ and $\mathrm{B}_{2}=\left[\left(\delta_{1}^{L}, \delta_{2}^{L}, \delta_{3}^{L}, \delta_{4}^{L} ; \mathrm{H}_{\mathrm{B}_{2}}^{L}\right)\right.$, $\left.\left(\delta_{1}^{U}, \delta_{2}^{U}, \delta_{3}^{U}, \delta_{4}^{U} ; \mathrm{H}_{\mathrm{B}_{2}}^{U}\right)\right]$ be two IT2FSs. Then, the operational laws between $B_{1}$ and $B_{2}$ are defined as follows:

(1) $\mathrm{B}_{1} \oplus \mathrm{B}_{2}$

$$
=\left[\begin{array}{c}
\left(\beta_{1}^{L}+\delta_{1}^{L}, \beta_{2}^{L}+\delta_{2}^{L}, \beta_{3}^{L}+\delta_{3}^{L}, \beta_{4}^{L}+\delta_{4}^{L} ; \min \left\{\mathrm{H}_{\mathrm{B}_{1}}^{L}, \mathrm{H}_{\mathrm{B}_{3}}^{L}\right\}\right), \\
\left(\beta_{1}^{U}+\delta_{1}^{U}, \beta_{2}^{U}+\delta_{2}^{U}, \beta_{3}^{U}+\delta_{3}^{U}, \beta_{4}^{U}+\delta_{4}^{U} ; \min \left\{\mathrm{H}_{\mathrm{B}_{1}}^{U}, \mathrm{H}_{\mathrm{B}_{2}}^{U}\right\}\right)
\end{array}\right]
$$

(2) $\mathrm{B}_{1} \odot \mathrm{B}_{2}$

$$
=\left[\begin{array}{c}
\left(\beta_{1}^{L}-\delta_{1}^{L}, \beta_{2}^{L}-\delta_{2}^{L}, \beta_{3}^{L}-\delta_{3}^{L}, \beta_{4}^{L}-\delta_{4}^{L} ; \min \left\{\mathrm{H}_{\mathrm{B}_{1}}^{L}, \mathrm{H}_{\mathrm{B}_{2}}^{L}\right\}\right), \\
\left(\beta_{1}^{U}-\delta_{1}^{U}, \beta_{2}^{U}-\delta_{2}^{U}, \beta_{3}^{U}-\delta_{3}^{U}, \beta_{4}^{U}-\delta_{4}^{U} ; \min \left\{\mathrm{H}_{\mathrm{B}_{1}}^{U}, \mathrm{H}_{\mathrm{B}_{2}}^{U}\right\}\right)
\end{array}\right]
$$

(3) $\mathrm{B}_{1} \otimes \mathrm{B}_{2}=\left[\begin{array}{c}\left(\beta_{1}^{L} \delta_{1}^{L}, \beta_{2}^{L} \delta_{2}^{L}, \beta_{3}^{L} \delta_{3}^{L}, \beta_{4}^{L} \delta_{4}^{L} ; \min \left\{\mathrm{H}_{\mathrm{B}_{1}}^{L}, \mathrm{H}_{\mathrm{B}_{2}}^{L}\right\}\right), \\ \left(\beta_{1}^{U} \delta_{1}^{U}, \beta_{2}^{U} \delta_{2}^{U}, \beta_{3}^{U} \delta_{3}^{U}, \beta_{4}^{U} \delta_{4}^{U} ; \min \left\{\mathrm{H}_{\mathrm{B}_{1}}^{U}, \mathrm{H}_{\mathrm{B}_{2}}^{U}\right\}\right)\end{array}\right]$

(4) $\mathrm{B}_{1} \oslash \mathrm{B}_{2}=\left[\begin{array}{l}\left(\frac{\beta_{1}^{L}}{\delta_{1}^{L}}, \frac{\beta_{2}^{L}}{\delta_{2}^{L}}, \frac{\beta_{3}^{L}}{\delta_{3}^{L}}, \frac{\beta_{4}^{L}}{\delta_{4}^{L}} ; \min \left\{\mathrm{H}_{\mathrm{B}_{1}}^{L}, \mathrm{H}_{\mathrm{B}_{2}}^{L}\right\}\right), \\ \left(\frac{\beta_{1}^{U}}{\delta_{1}^{U}}, \frac{\beta_{2}^{U}}{\delta_{2}^{U}}, \frac{\beta_{3}^{U}}{\delta_{3}^{U}}, \frac{\beta_{4}^{U}}{\delta_{4}^{U}} ; \min \left\{\mathrm{H}_{\mathrm{B}_{1}}^{U}, \mathrm{H}_{\mathrm{B}_{2}}^{U}\right\}\right)\end{array}\right]$

(5) $\gamma \mathrm{B}=\left[\left(\gamma \beta_{1}^{L}, \gamma \beta_{2}^{L}, \gamma \beta_{3}^{L}, \gamma \beta_{4}^{L} ; \mathrm{H}_{\mathrm{B}}^{L}\right),\left(\gamma \beta_{1}^{U}, \gamma \beta_{2}^{U}, \gamma \beta_{3}^{U}, \gamma \beta_{4}^{U} ; \mathrm{H}_{\mathrm{B}}^{U}\right)\right]$

(6) $\frac{\mathrm{B}}{\gamma}=\left[\left(\frac{\beta_{1}^{L}}{\gamma}, \frac{\beta_{2}^{L}}{\gamma}, \frac{\beta_{3}^{L}}{\gamma}, \frac{\beta_{4}^{L}}{\gamma} ; h_{\mathrm{A}}^{L}\right),\left(\frac{\beta_{1}^{U}}{\gamma}, \frac{\beta_{2}^{U}}{\gamma}, \frac{\beta_{3}^{U}}{\gamma}, \frac{\beta_{4}^{U}}{\gamma} ; h_{\mathrm{A}}^{U}\right)\right]$ $\gamma>0$

(7) $\mathrm{B}^{\gamma}=\left[\left(\left(\beta_{1}^{L}\right)^{\gamma},\left(\beta_{2}^{L}\right)^{\gamma},\left(\beta_{3}^{L}\right)^{\gamma},\left(\beta_{4}^{L}\right)^{\gamma} ; \mathrm{H}_{\mathrm{B}}^{L}\right)\right.$,

$$
\left.\left(\left(\beta_{1}^{U}\right)^{\gamma},\left(\beta_{2}^{U}\right)^{\gamma},\left(\beta_{3}^{U}\right)^{\gamma},\left(\beta_{4}^{U}\right)^{\gamma} ; \mathrm{H}_{\mathrm{B}}^{U}\right)\right]
$$


Definition 5 ([35]). Let $\mathrm{B}=\left(\mathrm{B}^{L}, \mathrm{~B}^{U}\right)$ be an IT2FS for any $c \in$ $[0,1]$. Then, the $c$-cut of $B$ is defined as follows:

$$
\mathrm{B}_{c}(\chi)=\eta \mathrm{A}_{c}^{L}(\phi)+(1-\eta) \mathrm{A}_{c}^{U}(\phi)
$$

where $\eta$ is a given coefficient, and $\eta \in[0,1]$.

Definition 6 ([35]). Let $\mathrm{B}=\left(\mathrm{B}^{L}, \mathrm{~B}^{U}\right)$ be an IT2FS for any $\chi \epsilon$ $[0,1]$. Then, $\mathrm{A}_{\chi}(\phi)$ can be defined as follows:

$$
\mathrm{A}_{\chi}(\phi)= \begin{cases}1, & \phi \in \mathrm{A}_{\chi}^{L}(\phi) \\ \eta, & \phi \notin \mathrm{A}_{\chi}^{L}(\phi) \text { and } \phi \in \mathrm{A}_{\chi}^{U}(\phi) \\ 0, & \phi \notin \mathrm{A}_{\chi}^{U}(\phi)\end{cases}
$$

Definition 7 ([35, 37]). Let $\mathrm{B}=\left[\left(\beta_{1}^{L}, \beta_{2}^{L}, \beta_{3}^{L}, \beta_{4}^{L} ; \mathrm{H}_{\mathrm{B}}^{L}\right)\right.$, $\left.\left(\beta_{1}^{U}, \beta_{2}^{U}, \beta_{3}^{U}, \beta_{4}^{U} ; \mathrm{H}_{\mathrm{B}}^{U}\right)\right]$ be an IT2FS. Then, the rank-based distance measure function (RDMF) between $B$ and $\tilde{\widetilde{1}}$ can be defined as follows:

$$
\begin{aligned}
& D(\mathrm{~B}, \tilde{\tilde{1}})=\int_{0}^{1}(1 \\
& \left.\quad-\frac{\int_{n}^{m} \min \left(\mathrm{A}_{\chi}(\phi), \widetilde{\tilde{1}}_{\chi}(\phi)\right) d \phi}{\int_{n}^{m}\left(\mathrm{~A}_{\chi}(\phi) \tilde{\tilde{1}}_{\chi}(\phi)\right)^{1 / 2} d \phi}\right) d \chi=1 \\
& -\beta_{4}^{L}-\eta\left(\beta_{1}^{L}-\beta_{1}^{U}+\beta_{4}^{U}-\beta_{4}^{L}\right) \\
& -\frac{1}{2 \mathrm{H}_{\mathrm{B}}^{U} \mathrm{H}_{\mathrm{B}}^{L}}\left[\mathrm { H } _ { \mathrm { B } } ^ { U } \left(\eta\left(\beta_{2}^{L}-\beta_{1}^{L}-\beta_{2}^{U}+\beta_{1}^{U}\right)\right.\right. \\
& \left.-\left(\beta_{4}^{L}-\beta_{3}^{L}-\beta_{2}^{L}+\beta_{1}^{L}\right)\right)-\mathrm{H}_{\mathrm{B}}^{L}\left(\beta_{4}^{U}-\beta_{3}^{U}-\beta_{4}^{L}\right. \\
& \left.\left.+\beta_{3}^{L}\right)\right]
\end{aligned}
$$

where $\tilde{\widetilde{1}}=[(1,1,1,1 ; 1),(1,1,1,1 ; 1)]$.

Generally, the value of parameter $\eta$ can denote the decision-maker's (DM's) preference in real MCDM problems. If the DM's attitude is negative, $\eta$ can be 1 ; if the DM's attitude is positive, $\eta$ can be 0 ; and if the DM's attitude is indifferent, $\eta$ can be 0.5 . In this paper, in order to reduce computational complexity, we assume that DM's attitude is indifferent in the practical decision-making (DM) environment, i.e., $\eta$ is 0.5 .

Definition $8([35,37])$. Let $\mathrm{B}_{1}$ and $\mathrm{B}_{2}$ be two IT2Ss. Then, the ranking of $\mathrm{B}_{1}$ and $\mathrm{B}_{2}$ based on RDMF can be defined as follows:
(1) If $D\left(\mathrm{~B}_{1}, \widetilde{\widetilde{1}}\right)>D\left(\mathrm{~B}_{2}, \widetilde{\widetilde{1}}\right)$, then $\mathrm{B}_{1}>\mathrm{B}_{2}$.

(2) If $D\left(\mathrm{~B}_{1}, \widetilde{\tilde{1}}\right)=D\left(\mathrm{~B}_{2}, \widetilde{\tilde{1}}\right)$, then $\mathrm{B}_{1} \sim \mathrm{B}_{2}$.

\subsection{PHM Operator}

Definition 9 ([20]). Let $\varepsilon=\left\{\varepsilon_{i} \mid i=1,2, \cdots n\right\}$ be a set of positive numbers, and $p, q>0$. Then, the HM operator of $\varepsilon=\left\{\varepsilon_{i} \mid i=1,2, \cdots n\right\}$ can be defined as follows:

$$
\begin{aligned}
& H M^{a, b}\left(\varepsilon_{1}, \varepsilon_{2}, \cdots, \varepsilon_{n}\right) \\
& =\left(\frac{2}{n(n+1)} \sum_{i=1}^{n} \sum_{j=i}^{n}{\varepsilon_{i}}^{p} \varepsilon_{j}^{q}\right)^{1 /(p+q)}
\end{aligned}
$$

Definition 10 ([21, 22]). Let $\varepsilon=\left\{\varepsilon_{i} \mid i=1,2, \cdots n\right\}$ be a set of positive numbers, which is divided into $\kappa$ clusters as $P_{1}, P_{2}, \cdots, P_{\kappa}$, where $P_{\gamma}=\left\{\varepsilon_{\gamma 1}, \varepsilon_{\gamma 2}, \cdots, \varepsilon_{\gamma \mid P_{\gamma}}\right\}(\gamma=$ $\left.1,2, \cdots,\left|P_{\gamma}\right|\right), \bigcup_{\gamma=1}^{\kappa} P_{\gamma}=\varepsilon, \sum_{\gamma=1}^{\kappa}\left|P_{\gamma}\right|=n$, and $\left|P_{\gamma}\right|$ represents the cardinality of $P_{\gamma}$. For any $p, q>0$, the partitioned HM (PHM) operator of $\varepsilon=\left\{\varepsilon_{i} \mid i=1,2, \cdots n\right\}$ can be defined as follows:

$$
\begin{aligned}
& \operatorname{PHM}^{p, q}\left(\varepsilon_{1}, \varepsilon_{2}, \cdots, \varepsilon_{n}\right) \\
& =\frac{1}{\kappa}\left(\sum_{\gamma=1}^{\kappa}\left(\frac{2}{\left|P_{\gamma}\right|\left(\left|P_{\gamma}\right|+1\right)} \sum_{i=1}^{\left|P_{\eta}\right|\left|P_{\gamma=i}\right|} \sum_{j i}^{p} \varepsilon_{\gamma j}^{p} \varepsilon^{q}\right)^{1 /(p+q)}\right)
\end{aligned}
$$

\section{IT2FPHM Operator and IT2FWPHM Operator}

Definition 11. Let $\mathrm{B}=\left\{\mathrm{B}_{i} \mid i=1,2, \cdots n\right\}$ be a collection of IT2FSs, which is divided into $\kappa$ clusters $P_{1}, P_{2}, \cdots, P_{\kappa}$, where $\mathrm{B}_{i}=\left[\left(\beta_{i 1}^{L}, \beta_{i 2}^{L}, \beta_{i 3}^{L}, \beta_{i 4}^{L} ; \mathrm{H}_{\mathrm{B}_{i}}^{L}\right),\left(\beta_{i 1}^{U}, \beta_{i 2}^{U}, \beta_{i 3}^{U}, \beta_{i 4}^{U} ; \mathrm{H}_{\mathrm{B}_{i}}^{U}\right)\right], \bigcup_{\gamma=1}^{\kappa} P_{\gamma}=$ $\mathrm{B}, \sum_{\gamma=1}^{\kappa}\left|P_{\gamma}\right|=n P_{\gamma}=\left\{\mathrm{B}_{\gamma 1}, \mathrm{~B}_{\gamma 2}, \cdots, \mathrm{B}_{\gamma \mid P_{\gamma}}\right\}\left(\gamma=1,2, \cdots,\left|P_{\gamma}\right|\right)$, and $\left|P_{\gamma}\right|$ represents the cardinality of $P_{\gamma}$. For any $p, q>0$, the IT2FPHM operator of $\mathrm{B}_{1}, \mathrm{~B}_{2}, \cdots$, and $\mathrm{B}_{n}$ can be defined as follows:

$$
\begin{array}{r}
I T 2 F P H M^{p, q}\left(\mathrm{~B}_{1}, \mathrm{~B}_{2}, \cdots, \mathrm{B}_{n}\right) \\
=\frac{1}{\kappa}\left(\sum _ { \gamma = 1 } ^ { \kappa } \left(\frac{2}{\left|P_{\gamma}\right|\left(\left|P_{\gamma}\right|+1\right)}\right.\right. \\
\left.\left.\quad \cdot \sum_{i=1}^{\left|P_{\gamma}\right|\left|P_{\gamma}\right|} \sum_{j=i} \mathrm{~B}_{\gamma i}^{p} \otimes \mathrm{B}_{\gamma j}{ }^{q}\right)^{1 /(p+q)}\right)
\end{array}
$$

Theorem 12. Let $\mathrm{B}=\left\{\mathrm{B}_{i} \mid i=1,2, \cdots n\right\}$ be a collection of IT2FSs, and $p, q>0$. The aggregated value of the IT2FPBM operator is still an IT2FS, and 


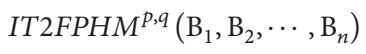

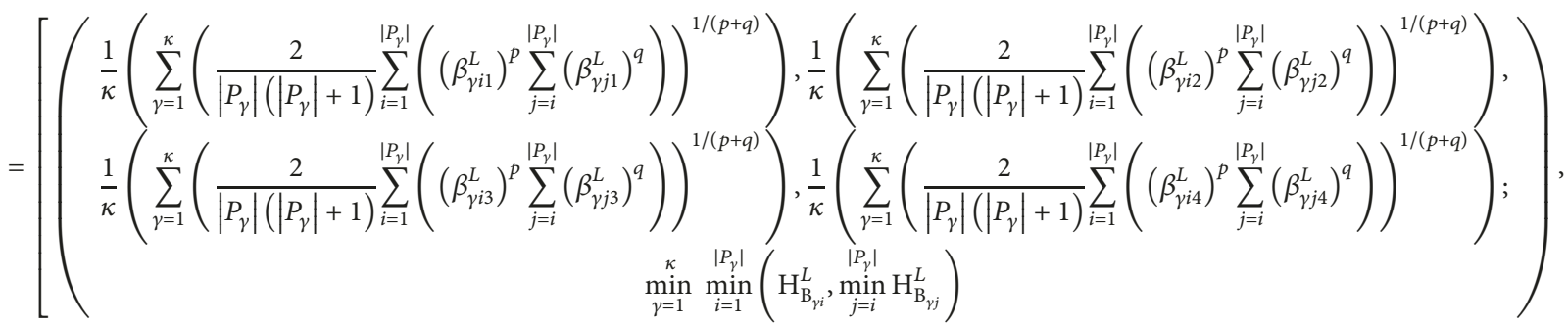

$$
\begin{aligned}
& \left(\begin{array}{c}
\frac{1}{\kappa}\left(\sum_{\gamma=1}^{\kappa}\left(\frac{2}{\left|P_{\gamma}\right|\left(\left|P_{\gamma}\right|+1\right)} \sum_{i=1}^{\left|P_{\gamma}\right|}\left(\left(\beta_{\gamma i 1}^{U}\right)^{p} \sum_{j=i}^{\left|P_{\gamma}\right|}\left(\beta_{\gamma j 1}^{U}\right)^{q}\right)\right)^{1 /(p+q)}\right), \frac{1}{\kappa}\left(\sum_{\gamma=1}^{\kappa}\left(\frac{2}{\left|P_{\gamma}\right|\left(\left|P_{\gamma}\right|+1\right)} \sum_{i=1}^{\left|P_{\gamma}\right|}\left(\left(\beta_{\gamma i 2}^{U}\right)^{p} \sum_{j=i}^{\left|P_{\gamma}\right|}\left(\beta_{\gamma j 2}^{U}\right)^{q}\right)\right)\right. \\
\frac{1}{\kappa}\left(\sum_{\gamma=1}^{\kappa}\left(\frac{2}{\left|P_{\gamma}\right|\left(\left|P_{\gamma}\right|+1\right)} \sum_{i=1}^{\left|P_{\gamma}\right|}\left(\left(\beta_{\gamma i 3}^{U}\right)^{p} \sum_{j=i}^{\left|P_{\gamma}\right|}\left(\beta_{\gamma j 3}^{U}\right)^{q}\right)\right)^{1 /(p+q)}\right), \frac{1}{\kappa}\left(\sum_{\gamma=1}^{\kappa}\left(\frac{2}{\left|P_{\gamma}\right|\left(\left|P_{\gamma}\right|+1\right)} \sum_{i=1}^{\left|P_{\gamma}\right|}\left(\left(\beta_{\gamma i 4}^{U}\right)^{p} \sum_{j=i}^{\left|P_{\gamma}\right|}\left(\beta_{\gamma j 4}^{U}\right)^{q}\right)\right)\right. \\
\substack{\kappa i n \\
\gamma=1}
\end{array}\right) ;
\end{aligned}
$$

Proof. Based on (11) and (13), we get

$$
\begin{gathered}
\mathrm{B}_{\gamma i}^{p}=\left[\left(\left(\beta_{\gamma i 1}^{L}\right)^{p},\left(\beta_{\gamma i 2}^{L}\right)^{p},\left(\beta_{\gamma i 3}^{L}\right)^{p},\left(\beta_{\gamma i 4}^{L}\right)^{p} ; \mathrm{H}_{\mathrm{B}_{i}}^{L}\right),\right. \\
\left.\left(\left(\beta_{\gamma i 1}^{U}\right)^{p},\left(\beta_{\gamma i 2}^{U}\right)^{p},\left(\beta_{\gamma i 3}^{U}\right)^{p},\left(\beta_{\gamma i 4}^{U}\right)^{p} ; \mathrm{H}_{\mathrm{B}_{i}}^{U}\right)\right]
\end{gathered}
$$

and

$$
\begin{gathered}
\left(\mathrm{B}_{\gamma i}^{L}\right)^{p} \sum_{j=i}^{\left|P_{\gamma}\right|}\left(\mathrm{B}_{\gamma j}^{U}\right)^{q}=\left[\left(\left(\beta_{\gamma i 1}^{L}\right)^{p} \sum_{j=i}^{\left|P_{\gamma}\right|}\left(\beta_{\gamma j 1}^{L}\right)^{q},\left(\beta_{\gamma i 2}^{L}\right)^{p}\right.\right. \\
\cdot \sum_{j=i}^{\left|P_{\gamma}\right|}\left(\beta_{\gamma j 2}^{L}\right)^{q},\left(\beta_{\gamma i 3}^{L}\right)^{p} \sum_{j=i}^{\left|P_{\gamma}\right|}\left(\beta_{\gamma j 3}^{L}\right)^{q},\left(\beta_{\gamma i 4}^{L}\right)^{p}
\end{gathered}
$$

$\left.\cdot \sum_{j=i}^{\left|P_{\gamma}\right|}\left(\beta_{\gamma j 4}^{L}\right)^{q} ; \min \left(\mathrm{H}_{\mathrm{B}_{\gamma i}}^{L}, \min _{j=i}^{\left|P_{\gamma}\right|} \mathrm{H}_{\mathrm{B}_{\gamma j}}^{L}\right)\right),\left(\left(\beta_{\gamma i 1}^{U}\right)^{p}\right.$

$\cdot \sum_{j=i}^{\left|P_{\gamma}\right|}\left(\beta_{\gamma j 1}^{U}\right)^{q},\left(\beta_{\gamma i 2}^{U}\right)^{p} \sum_{j=i}^{\left|P_{\gamma}\right|}\left(\beta_{\gamma j 2}^{U}\right)^{q},\left(\beta_{\gamma i 3}^{U}\right)^{p}$

$\cdot \sum_{j=i}^{\left|P_{\gamma}\right|}\left(\beta_{\gamma j 3}^{U}\right)^{q},\left(\beta_{\gamma i 4}^{U}\right)^{p}$

$\left.\left.\cdot \sum_{j=i}^{\left|P_{\gamma}\right|}\left(\beta_{\gamma j 4}^{U}\right)^{q} ; \min \left(\mathrm{H}_{\mathrm{B}_{\gamma i}}^{U}, \min _{j=i}^{\left|P_{\gamma}\right|} \mathrm{H}_{\mathrm{B}_{\gamma j}}^{U}\right)\right)\right]$

Then,

$$
\begin{aligned}
& \frac{2}{\left|P_{\gamma}\right|\left(\left|P_{\gamma}\right|+1\right)} \sum_{i=1}^{\left|P_{\gamma}\right|\left|P_{\gamma}\right|} \sum_{j=i} \mathrm{~B}_{\gamma i}^{p} \otimes \mathrm{B}_{\gamma j}{ }^{q} \\
& =\left[\begin{array}{c}
\frac{2}{\left|P_{\gamma}\right|\left(\left|P_{\gamma}\right|+1\right)} \sum_{i=1}^{\left|P_{\gamma}\right|}\left(\beta_{\gamma i 1}^{L}\right)^{p} \sum_{j=i}^{\left|P_{\gamma}\right|}\left(\beta_{\gamma j 1}^{L}\right)^{q}, \frac{2}{\left|P_{\gamma}\right|\left(\left|P_{\gamma}\right|+1\right)} \sum_{i=1}^{\left|P_{\gamma}\right|}\left(\beta_{\gamma i 2}^{L}\right)^{p} \sum_{j=i}^{\left|P_{\gamma}\right|}\left(\beta_{\gamma j 2}^{L}\right)^{q}, \\
\frac{2}{\left|P_{\gamma}\right|\left(\left|P_{\gamma}\right|+1\right)} \sum_{i=1}^{\left|P_{\gamma}\right|}\left(\beta_{\gamma i 3}^{L}\right)^{p} \sum_{j=i}^{\left|P_{\gamma}\right|}\left(\beta_{\gamma j 3}^{L}\right)^{q}, \frac{2}{\left|P_{\gamma}\right|\left(\left|P_{\gamma}\right|+1\right)} \sum_{i=1}^{\left|P_{\gamma}\right|}\left(\beta_{\gamma i 4}^{L}\right)^{p} \sum_{j=i}^{\left|P_{\gamma}\right|}\left(\beta_{\gamma j 4}^{L}\right)^{q} ; \min _{i=1}\left(\mathrm{H}_{\mathrm{B}_{\gamma i}}^{L}, \min _{j=i}^{\left|P_{\gamma}\right|} \mathrm{H}_{\mathrm{B}_{\gamma j}}^{L}\right)
\end{array}\right), \\
& \left.\left.\left(\begin{array}{c}
\frac{2}{\left|P_{\gamma}\right|\left(\left|P_{\gamma}\right|+1\right)} \sum_{i=1}^{\left|P_{\gamma}\right|}\left(\beta_{\gamma i 1}^{U}\right)^{p} \sum_{j=i}^{\left|P_{\gamma}\right|}\left(\beta_{\gamma j 1}^{U}\right)^{q}, \frac{2}{\left|P_{\gamma}\right|\left(\left|P_{\gamma}\right|+1\right)} \sum_{i=1}^{\left|P_{\gamma}\right|}\left(\beta_{\gamma i 2}^{U}\right)^{p} \sum_{j=i}^{\left|P_{\gamma}\right|}\left(\beta_{\gamma j 2}^{U}\right)^{q}, \\
\frac{2}{\left|P_{\gamma}\right|\left(\left|P_{\gamma}\right|+1\right)} \sum_{i=1}^{\left|P_{\gamma}\right|}\left(\beta_{\gamma i 3}^{U}\right)^{p} \sum_{j=i}^{\left|P_{\gamma}\right|}\left(\beta_{\gamma j 3}^{U}\right)^{q}, \frac{2}{\left|P_{\gamma}\right|\left(\left|P_{\gamma}\right|+1\right)} \sum_{i=1}^{\left|P_{\gamma}\right|}\left(\beta_{\gamma i 4}^{U}\right)^{p} \sum_{j=i}^{\left|P_{\gamma}\right|}\left(\beta_{\gamma j 4}^{U}\right)^{q} ; \min _{i=1}\left(\mathrm{H}_{\mathrm{B}_{\gamma i}}^{U}, \min _{j=i}^{\left|P_{\gamma}\right|} \mathrm{H}_{\mathrm{B}_{\gamma j}}^{U}\right)
\end{array}\right)\right)\right]
\end{aligned}
$$


Finally, we can obtain

$$
\begin{aligned}
& \frac{1}{\kappa}\left(\sum_{\gamma=1}^{\kappa}\left(\frac{2}{\left|P_{\gamma}\right|\left(\left|P_{\gamma}\right|+1\right)} \sum_{i=1}^{\left|P_{\gamma}\right| P_{j=i} \mid} \mathrm{B}_{\gamma i}{ }^{p} \otimes \mathrm{B}_{\gamma j}{ }^{q}\right)^{1 /(p+q)}\right)
\end{aligned}
$$

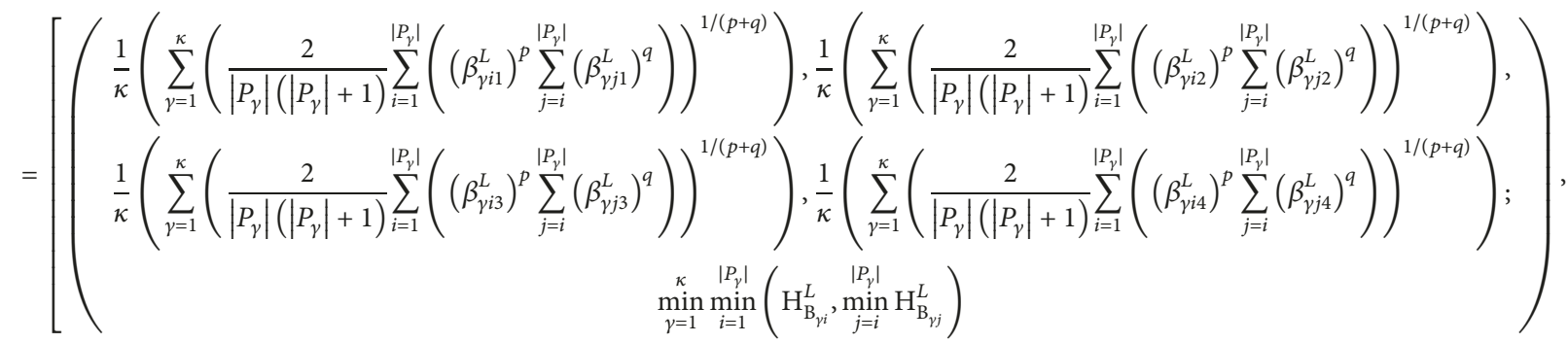

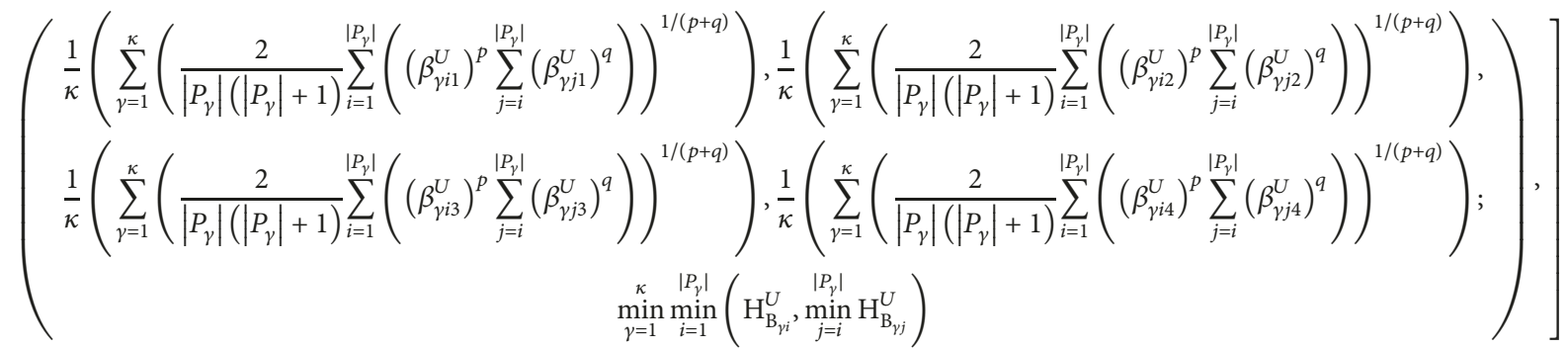

Theorem 13 (idempotency). Let $\mathrm{B}=\left\{\mathrm{B}_{i} \mid i=1,2, \cdots n\right\}$ be a collection of IT2FSs. If all $\mathrm{A}_{i}(i=1,2, \cdots n)$ are equal, that $i s, \mathrm{~B}_{i}=\mathrm{B}=\left[\left(\beta_{1}^{L}, \beta_{2}^{L}, \beta_{3}^{L}, \beta_{4}^{L} ; \mathrm{H}_{\mathrm{B}}^{L}\right),\left(\beta_{1}^{U}, \beta_{2}^{U}, \beta_{3}^{U}, \beta_{4}^{U} ; \mathrm{H}_{\mathrm{B}}^{U}\right)\right](i=$ $1,2, \cdots, n)$, then

$$
\operatorname{IT} 2 F P H M^{a, b}\left(\mathrm{~B}_{1}, \mathrm{~B}_{2}, \cdots, \mathrm{B}_{n}\right)=\mathrm{B} .
$$

Proof.

$$
\begin{aligned}
& \frac{1}{\kappa}\left(\sum _ { \gamma = 1 } ^ { \kappa } \left(\frac{2}{\left|P_{\gamma}\right|\left(\left|P_{\gamma}\right|+1\right)}\right.\right. \\
& \left.\left.\cdot \sum_{i=1}^{\left|P_{\gamma}\right|}\left(\left(\beta_{\gamma i 1}^{L}\right)^{p} \sum_{j=i}^{\left|P_{\gamma}\right|}\left(\beta_{\gamma j 1}^{L}\right)^{q}\right)\right)^{1 /(p+q)}\right) \\
& =\frac{1}{\kappa}\left(\sum _ { \gamma = 1 } ^ { \kappa } \left(\frac{2}{\left|P_{\gamma}\right|\left(\left|P_{\gamma}\right|+1\right)} \times \frac{\left|P_{\gamma}\right|\left(\left|P_{\gamma}\right|+1\right)}{2}\right.\right. \\
& \left.\left.\times\left(\beta_{1}^{L}\right)^{p+q}\right)^{1 /(p+q)}\right)=\frac{1}{\kappa}\left(\sum_{\gamma=1}^{\kappa} \beta_{1}^{L}\right)=\beta_{1}^{L}
\end{aligned}
$$

\section{Similarity, we can obtain}

$$
\begin{aligned}
& \frac{1}{\mathcal{\kappa}}\left(\sum _ { \gamma = 1 } ^ { \kappa } \left(\frac{2}{\left|P_{\gamma}\right|\left(\left|P_{\gamma}\right|+1\right)}\right.\right. \\
& \left.\left.\quad \cdot \sum_{i=1}^{\left|P_{\gamma}\right|}\left(\left(\beta_{\gamma i 2}^{L}\right)^{p} \sum_{j=i}^{\left|P_{\gamma}\right|}\left(\beta_{\gamma j 2}^{L}\right)^{q}\right)\right)^{1 /(p+q)}\right)=\beta_{2}^{L},
\end{aligned}
$$

$$
\begin{aligned}
& \frac{1}{\kappa}\left(\sum _ { \gamma = 1 } ^ { \kappa } \left(\frac{2}{\left|P_{\gamma}\right|\left(\left|P_{\gamma}\right|+1\right)}\right.\right. \\
&\left.\left.\cdot \sum_{i=1}^{\left|P_{\gamma}\right|}\left(\left(\beta_{\gamma i 3}^{L}\right)^{p} \sum_{j=i}^{\left|P_{\gamma}\right|}\left(\beta_{\gamma j 3}^{L}\right)^{q}\right)\right)^{1 /(p+q)}\right)=\beta_{3}^{L}
\end{aligned}
$$$$
\frac{1}{\kappa}\left(\sum _ { \gamma = 1 } ^ { \kappa } \left(\frac{2}{\left|P_{\gamma}\right|\left(\left|P_{\gamma}\right|+1\right)}\right.\right.
$$

$$
\left.\left.\cdot \sum_{i=1}^{\left|P_{\gamma}\right|}\left(\left(\beta_{\gamma i 4}^{L}\right)^{p} \sum_{j=i}^{\left|P_{\gamma}\right|}\left(\beta_{\gamma j 4}^{L}\right)^{q}\right)\right)^{1 /(p+q)}\right)=\beta_{4}^{L},
$$

$$
\frac{1}{\kappa}\left(\sum _ { \gamma = 1 } ^ { \kappa } \left(\frac{2}{\left|P_{\gamma}\right|\left(\left|P_{\gamma}\right|+1\right)}\right.\right.
$$




$$
\begin{gathered}
\left.\left.\cdot \sum_{i=1}^{\left|P_{\gamma}\right|}\left(\left(\beta_{\gamma i 1}^{U}\right)^{p} \sum_{j=i}^{\left|P_{\gamma}\right|}\left(\beta_{\gamma j 1}^{U}\right)^{q}\right)\right)^{1 /(p+q)}\right)=\beta_{1}^{U} \\
\frac{1}{\kappa}\left(\sum_{\gamma=1}^{\kappa}\left(\frac{2}{\left|P_{\gamma}\right|\left(\left|P_{\gamma}\right|+1\right)}\right)=\beta_{2}^{U},\right. \\
\left.\left.\cdot \sum_{i=1}^{\left|P_{\gamma}\right|}\left(\left(\beta_{\gamma i 2}^{U}\right)^{p} \sum_{j=i}^{\left|P_{\gamma}\right|}\left(\beta_{\gamma j 2}^{U}\right)^{q}\right)\right)^{1 /(p+q)}\right) \\
\frac{1}{\kappa}\left(\sum_{\gamma=1}^{\kappa}\left(\frac{2}{\left|P_{\gamma}\right|\left(\left|P_{\gamma}\right|+1\right)}\right)=\beta_{3}^{U}\right. \\
\left.\quad \sum_{i=1}^{\left|P_{\gamma}\right|}\left(\left(\beta_{\gamma i 3}^{U}\right)^{p} \sum_{j=i}^{\left|P_{\gamma}\right|}\left(\beta_{\gamma j 3}^{U}\right)^{q}\right)\right)^{1 /(p+q)}
\end{gathered}
$$

$$
\begin{aligned}
& \frac{1}{\kappa}\left(\sum _ { \gamma = 1 } ^ { \kappa } \left(\frac{2}{\left|P_{\gamma}\right|\left(\left|P_{\gamma}\right|+1\right)}\right.\right. \\
& \left.\left.\quad \cdot \sum_{i=1}^{\left|P_{\gamma}\right|}\left(\left(\beta_{\gamma i 4}^{U}\right)^{p} \sum_{j=i}^{\left|P_{\gamma}\right|}\left(\beta_{\gamma j 4}^{U}\right)^{q}\right)\right)^{1 /(p+q)}\right)=\beta_{4}^{U}
\end{aligned}
$$

In addition, $\min _{\gamma=1}^{\kappa} \min _{i=1}^{\left|P_{\gamma}\right|}\left(\mathrm{H}_{\mathrm{B}_{\gamma i}}^{L}, \min _{j=i}^{\left|P_{\gamma}\right|} \mathrm{H}_{\mathrm{B}_{\gamma j}}^{L}\right)=\mathrm{H}_{\mathrm{B}}^{L} \min _{\gamma=1}^{\kappa}$ $\min _{i=1}^{\left|P_{\gamma}\right|}\left(\mathrm{H}_{\mathrm{B}_{\gamma i}}^{U}, \min _{j=i}^{\left|P_{\gamma}\right|} \mathrm{H}_{\mathrm{B}_{\gamma j}}^{U}\right)=\mathrm{H}_{\mathrm{B}}^{U}$.

B.

Therefore, we can obtain $\operatorname{IT}_{2} \operatorname{FPHM}^{a, b}\left(\mathrm{~B}_{1}, \mathrm{~B}_{2}, \cdots, \mathrm{B}_{n}\right)=$

Theorem 14 (commutativity). Let $\mathrm{B}=\left\{\mathrm{B}_{i} \mid i=1,2, \cdots n\right\}$ and $\mathrm{B}^{\prime}=\left\{\mathrm{B}_{i}^{\prime} \mid i=1,2, \cdots n\right\}$ be two collections of IT2FSs. If $B_{i}^{\prime}=\left[\left(\beta_{1}^{L^{\prime}}, \beta_{2}^{L^{\prime}}, \beta_{3}^{L^{\prime}}, \beta_{4}^{L^{\prime}} ; \mathrm{H}_{\mathrm{B}}^{L^{\prime}}\right),\left(\beta_{1}^{U^{\prime}}, \beta_{2}^{U^{\prime}}, \beta_{3}^{U^{\prime}}, \beta_{4}^{U^{\prime}} ; \mathrm{H}_{\mathrm{B}}^{U^{\prime}}\right)\right](i=$ $1,2, \cdots, n)$ is any permutation of $\mathrm{B}_{i}=\left[\left(\beta_{1}^{L}, \beta_{2}^{L}\right.\right.$, $\left.\left.\beta_{3}^{L}, \beta_{4}^{L} ; \mathrm{H}_{\mathrm{B}}^{L}\right),\left(\beta_{1}^{U}, \beta_{2}^{U}, \beta_{3}^{U}, \beta_{4}^{U} ; \mathrm{H}_{\mathrm{B}}^{U}\right)\right](i=1,2, \cdots, n)$ then

$$
\begin{aligned}
& I T 2 F_{P H M^{p, q}}\left(\mathrm{~B}_{1}, \mathrm{~B}_{2}, \cdots, \mathrm{B}_{n}\right) \\
& \quad=\operatorname{IT} 2 F P H M^{p, q}\left(\mathrm{~B}_{1}{ }^{\prime}, \mathrm{B}_{2}{ }^{\prime}, \cdots, \mathrm{B}_{n}{ }^{\prime}\right) .
\end{aligned}
$$

Proof. Based on (35), we can get

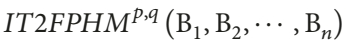

$$
\begin{aligned}
& =\left[\begin{array}{c}
\frac{1}{\kappa}\left(\sum_{\gamma=1}^{\kappa}\left(\frac{2}{\left|P_{\gamma}\right|\left(\left|P_{\gamma}\right|+1\right)} \sum_{i=1}^{\left|P_{\gamma}\right|}\left(\left(\beta_{\gamma i 1}^{L}\right)^{p} \sum_{j=i}^{\left|P_{\gamma}\right|}\left(\beta_{\gamma j 1}^{L}\right)^{q}\right)\right)^{1 /(p+q)}\right), \frac{1}{\kappa}\left(\sum_{\gamma=1}^{\kappa}\left(\frac{2}{\left|P_{\gamma}\right|\left(\left|P_{\gamma}\right|+1\right)} \sum_{i=1}^{\left|P_{\gamma}\right|}\left(\left(\beta_{\gamma i 2}^{L}\right)^{p} \sum_{j=i}^{\left|P_{\gamma}\right|}\left(\beta_{\gamma j 2}^{L}\right)^{q}\right)\right)\right. \\
\frac{1}{\kappa}\left(\sum_{\gamma=1}^{\kappa}\left(\frac{2}{\left|P_{\gamma}\right|\left(\left|P_{\gamma}\right|+1\right)} \sum_{i=1}^{\left|P_{\gamma}\right|}\left(\left(\beta_{\gamma i 3}^{L}\right)^{p} \sum_{j=i}^{\left|P_{\gamma}\right|}\left(\beta_{\gamma j 3}^{L}\right)^{q}\right)\right)^{1 /(p+q)}\right), \frac{1}{\kappa}\left(\sum_{\gamma=1}^{\kappa}\left(\frac{2}{\left|P_{\gamma}\right|\left(\left|P_{\gamma}\right|+1\right)} \sum_{i=1}^{\left|P_{\gamma}\right|}\left(\left(\beta_{\gamma i 4}^{L}\right)^{p} \sum_{j=i}^{\left|P_{\gamma}\right|}\left(\beta_{\gamma j 4}^{L}\right)^{q}\right)\right)\right. \\
\min _{\gamma=1}^{\kappa} \min _{i=1}^{\left|P_{\gamma}\right|}\left(\mathrm{H}_{\mathrm{B}_{\gamma i}}^{L}, \min _{j=i}^{\left|P_{\gamma}\right|} \mathrm{H}_{\mathrm{B}_{\gamma j}}^{L}\right)
\end{array}\right] ; \\
& \left(\begin{array}{c}
\frac{1}{\kappa}\left(\sum_{\gamma=1}^{\kappa}\left(\frac{2}{\left|P_{\gamma}\right|\left(\left|P_{\gamma}\right|+1\right)} \sum_{i=1}^{\left|P_{\gamma}\right|}\left(\left(\beta_{\gamma i 1}^{U}\right)^{p} \sum_{j=i}^{\left|P_{\gamma}\right|}\left(\beta_{\gamma j 1}^{U}\right)^{q}\right)\right)^{1 /(p+q)}\right), \frac{1}{\kappa}\left(\sum_{\gamma=1}^{\kappa}\left(\frac{2}{\left|P_{\gamma}\right|\left(\left|P_{\gamma}\right|+1\right)} \sum_{i=1}^{\left|P_{\gamma}\right|}\left(\left(\beta_{\gamma i 2}^{U}\right)^{p} \sum_{j=i}^{\left|P_{\gamma}\right|}\left(\beta_{\gamma j 2}^{U}\right)^{q}\right)\right)\right. \\
\frac{1}{\kappa}\left(\sum_{\gamma=1}^{\kappa}\left(\frac{2}{\left|P_{\gamma}\right|\left(\left|P_{\gamma}\right|+1\right)} \sum_{i=1}^{\left|P_{\gamma}\right|}\left(\left(\beta_{\gamma i 3}^{U}\right)^{p} \sum_{j=i}^{\left|P_{\gamma}\right|}\left(\beta_{\gamma j 3}^{U}\right)^{q}\right)\right)^{1 /(p+q)}\right), \frac{1}{\kappa}\left(\sum_{\gamma=1}^{\kappa}\left(\frac{2}{\left|P_{\gamma}\right|\left(\left|P_{\gamma}\right|+1\right)} \sum_{i=1}^{\left|P_{\gamma}\right|}\left(\left(\beta_{\gamma i 4}^{U}\right)^{p} \sum_{j=i}^{\left|P_{\gamma}\right|}\left(\beta_{\gamma j 4}^{U}\right)^{q}\right)\right)\right. \\
\min _{\gamma=1}^{\kappa} \min _{i=1}^{\left|P_{\gamma}\right|}\left(\mathrm{H}_{\mathrm{B}_{\gamma i}}^{U}, \min _{j=i}^{\left|P_{\gamma}\right|} \mathrm{H}_{\mathrm{B}_{\gamma j}}^{U}\right)
\end{array}\right]
\end{aligned}
$$

$\operatorname{IT} 2 F P H M^{p, q}\left(\mathrm{~B}_{1}{ }^{\prime}, \mathrm{B}_{2}{ }^{\prime}, \cdots, \mathrm{B}_{n}{ }^{\prime}\right)$

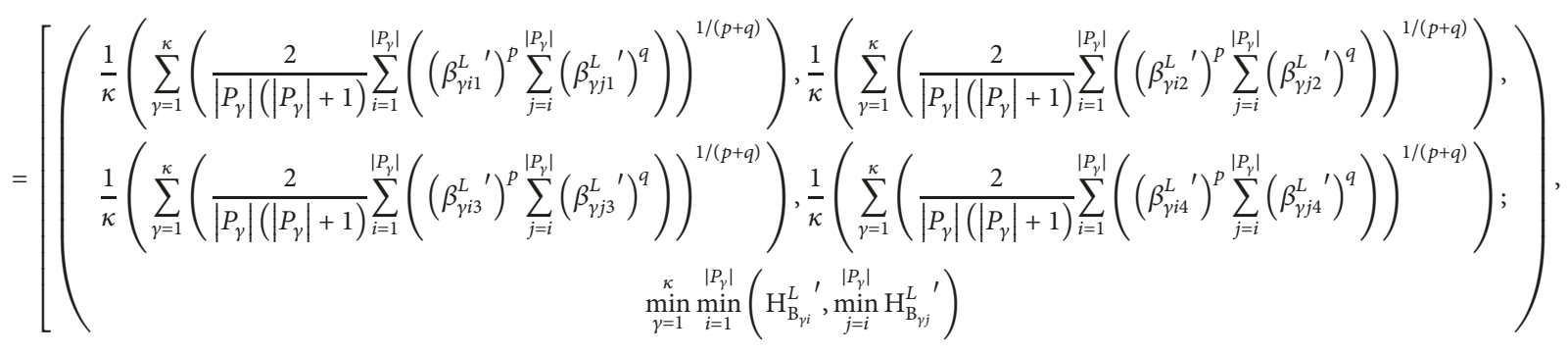




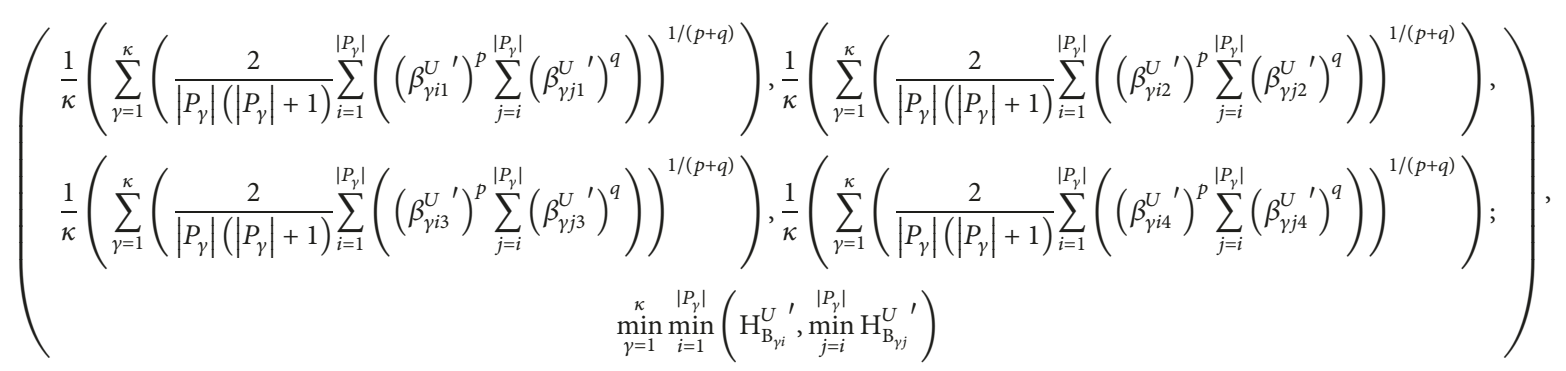

Because $\mathrm{B}_{i}{ }^{\prime}=\left[\left(\beta_{1}^{L^{\prime}}, \beta_{2}^{L^{\prime}}, \beta_{3}^{L^{\prime}}, \beta_{4}^{L^{\prime}} ; \mathrm{H}_{\mathrm{B}}^{L^{\prime}}\right),\left(\beta_{1}^{U^{\prime}}, \beta_{2}^{U^{\prime}}, \beta_{3}^{U^{\prime}}, \beta_{4}^{U^{\prime}}\right.\right.$; $\left.\left.\mathrm{H}_{\mathrm{B}}^{U^{\prime}}\right)\right](i=1,2, \cdots, n)$ is any permutation of $\mathrm{B}_{i}=$ $\left[\left(\beta_{1}^{L}, \beta_{2}^{L}, \beta_{3}^{L}, \beta_{4}^{L} ; \mathrm{H}_{\mathrm{B}}^{L}\right),\left(\beta_{1}^{U}, \beta_{2}^{U}, \beta_{3}^{U}, \beta_{4}^{U} ; \mathrm{H}_{\mathrm{B}}^{U}\right)\right](i=1,2, \cdots, n)$, obviously, we can obtain IT2FPHM IT,q $^{p}\left(\mathrm{~B}_{1}, \mathrm{~B}_{2}, \cdots, \mathrm{B}_{n}\right)=$ $\operatorname{IT} 2$ FPHM $^{p, q}\left(\mathrm{~B}_{1}{ }^{\prime}, \mathrm{B}_{2}{ }^{\prime}, \cdots, \mathrm{B}_{n}{ }^{\prime}\right)$.
In the following, we provide a few specific cases of the presented IT2FPHM operator with respect to the parameters $p$ and $q$.

(1) When $q=0$, we can obtain

$$
\begin{aligned}
& I T 2 F P H M^{p, 0}\left(\mathrm{~B}_{1}, \mathrm{~B}_{2}, \cdots, \mathrm{B}_{n}\right) \\
& =\left[\begin{array}{c}
\frac{1}{\kappa}\left(\sum_{\gamma=1}^{\kappa}\left(\frac{2}{\left|P_{\gamma}\right|\left(\left|P_{\gamma}\right|+1\right)} \sum_{i=1}^{\left|P_{\gamma}\right|}\left(\left|P_{\gamma}\right|+1-i\right)\left(\beta_{\gamma i 1}^{L}\right)^{P}\right)^{1 / p}\right), \frac{1}{\kappa}\left(\sum_{\gamma=1}^{\kappa}\left(\frac{2}{\left|P_{\gamma}\right|\left(\left|P_{\gamma}\right|+1\right)} \sum_{i=1}^{\left|P_{\gamma}\right|}\left(\left|P_{\gamma}\right|+1-i\right)\left(\beta_{\gamma i 2}^{L}\right)^{P}\right)^{1 / p}\right), \\
\frac{1}{\kappa}\left(\sum_{\gamma=1}^{\kappa}\left(\frac{2}{\left|P_{\gamma}\right|\left(\left|P_{\gamma}\right|+1\right)} \sum_{i=1}^{\left|P_{\gamma}\right|}\left(\left|P_{\gamma}\right|+1-i\right)\left(\beta_{\gamma i 3}^{L}\right)^{P}\right)^{1 / p}\right), \frac{1}{\kappa}\left(\sum_{\gamma=1}^{\kappa}\left(\frac{2}{\left|P_{\gamma}\right|\left(\left|P_{\gamma}\right|+1\right)} \sum_{i=1}^{\left|P_{\gamma}\right|}\left(\left|P_{\gamma}\right|+1-i\right)\left(\beta_{\gamma i 4}^{L}\right)^{P}\right)^{1 / p}\right) ; \min _{\gamma=1}^{\kappa} \min _{i=1}^{\left|P_{\gamma}\right|}\left(\mathrm{H}_{\mathrm{B}_{\gamma i}}^{L}, \min _{j=i}^{\left|P_{\gamma}\right|} \mathrm{H}_{\mathrm{B}_{\gamma j}}^{L}\right)
\end{array}\right), \\
& \left(\begin{array}{c}
\frac{1}{\kappa}\left(\sum_{\gamma=1}^{\kappa}\left(\frac{2}{\left|P_{\gamma}\right|\left(\left|P_{\gamma}\right|+1\right)} \sum_{i=1}^{\left|P_{\gamma}\right|}\left(\left|P_{\gamma}\right|+1-i\right)\left(\beta_{\gamma i 1}^{U}\right)^{P}\right)^{1 / p}\right), \frac{1}{\kappa}\left(\sum_{\gamma=1}^{\kappa}\left(\frac{2}{\left|P_{\gamma}\right|\left(\left|P_{\gamma}\right|+1\right)} \sum_{i=1}^{\left|P_{\gamma}\right|}\left(\left|P_{\gamma}\right|+1-i\right)\left(\beta_{\gamma i 2}^{U}\right)^{P}\right)^{1 / p}\right), \\
\frac{1}{\kappa}\left(\sum_{\gamma=1}^{\kappa}\left(\frac{2}{\left|P_{\gamma}\right|\left(\left|P_{\gamma}\right|+1\right)} \sum_{i=1}^{\left|P_{\gamma}\right|}\left(\left|P_{\gamma}\right|+1-i\right)\left(\beta_{\gamma i 3}^{U}\right)^{P}\right)^{1 / p}\right), \frac{1}{\kappa}\left(\sum_{\gamma=1}^{\kappa}\left(\frac{2}{\left|P_{\gamma}\right|\left(\left|P_{\gamma}\right|+1\right)} \sum_{i=1}^{\left|P_{\gamma}\right|}\left(\left|P_{\gamma}\right|+1-i\right)\left(\beta_{\gamma i 4}^{U}\right)^{P}\right)^{1 / p}\right) ; \min _{\gamma=1}^{\kappa} \min _{i=1}^{\left|P_{\gamma}\right|}\left(\begin{array}{c}
\left.\mathrm{H}_{\mathrm{B}_{\gamma i}}^{U}, \min _{j=i}^{\left|P_{\gamma}\right|} \mathrm{H}_{\mathrm{B}_{\gamma j}}^{U}\right)
\end{array}\right),
\end{array}\right.
\end{aligned}
$$

(2) When $P=0$, we can obtain

$$
\begin{aligned}
& \operatorname{IT} 2 F P H M^{0, q}\left(\mathrm{~B}_{1}, \mathrm{~B}_{2}, \cdots, \mathrm{B}_{n}\right) \\
& =\left[\begin{array}{c}
\frac{1}{\kappa}\left(\sum_{\gamma=1}^{\kappa}\left(\frac{2}{\left|P_{\gamma}\right|\left(\left|P_{\gamma}\right|+1\right)} \sum_{i=1}^{\left|P_{\gamma}\right|} i\left(\beta_{\gamma i 1}^{L}\right)^{q}\right)^{1 / q}\right), \frac{1}{\kappa}\left(\sum_{\gamma=1}^{\kappa}\left(\frac{2}{\left|P_{\gamma}\right|\left(\left|P_{\gamma}\right|+1\right)} \sum_{i=1}^{\left|P_{\gamma}\right|} i\left(\beta_{\gamma i 2}^{L}\right)^{q}\right)^{1 / q}\right), \frac{1}{\kappa}\left(\sum_{\gamma=1}^{\kappa}\left(\frac{2}{\left|P_{\gamma}\right|\left(\left|P_{\gamma}\right|+1\right)} \sum_{i=1}^{\left|P_{\gamma}\right|} i\left(\beta_{\gamma i 3}^{L}\right)^{q}\right)^{1 / q}\right. \\
\frac{1}{\kappa}\left(\sum_{\gamma=1}^{\kappa}\left(\frac{2}{\left|P_{\gamma}\right|\left(\left|P_{\gamma}\right|+1\right)} \sum_{i=1}^{\left|P_{\gamma}\right|} i\left(\beta_{\gamma i 4}^{L}\right)^{q}\right)^{1 / q}\right) ; \min _{\gamma=1}^{\kappa} \min _{i=1}^{\left|P_{\gamma}\right|}\left(\mathrm{H}_{\mathrm{B}_{\gamma i}}^{L}, \min _{j=i}^{\left|P_{\gamma}\right|} \mathrm{H}_{\mathrm{B}_{\gamma j}}^{L}\right)
\end{array}\right], \\
& \left(\begin{array}{c}
\frac{1}{\kappa}\left(\sum_{\gamma=1}^{\kappa}\left(\frac{2}{\left|P_{\gamma}\right|\left(\left|P_{\gamma}\right|+1\right)} \sum_{i=1}^{\left|P_{\gamma}\right|} i\left(\beta_{\gamma i 1}^{U}\right)^{p}\right)^{1 / q}\right), \frac{1}{\kappa}\left(\sum_{\gamma=1}^{\kappa}\left(\frac{2}{\left|P_{\gamma}\right|\left(\left|P_{\gamma}\right|+1\right)} \sum_{i=1}^{\left|P_{\gamma}\right|} i\left(\beta_{\gamma i 2}^{U}\right)^{p}\right)^{1 / q}\right), \frac{1}{\kappa}\left(\sum_{\gamma=1}^{\kappa}\left(\frac{2}{\left|P_{\gamma}\right|\left(\left|P_{\gamma}\right|+1\right)} \sum_{i=1}^{\left|P_{\gamma}\right|} i\left(\beta_{\gamma i 3}^{U}\right)^{p}\right)^{1 / q}\right), \\
\frac{1}{\kappa}\left(\sum_{\gamma=1}^{\kappa}\left(\frac{2}{\left|P_{\gamma}\right|\left(\left|P_{\gamma}\right|+1\right)} \sum_{i=1}^{\left|P_{\gamma}\right|} i\left(\beta_{\gamma i 4}^{U}\right)^{p}\right)^{1 / q}\right) ; \underset{\gamma=1}{\kappa} \min _{i=1}^{\left|P_{\gamma}\right|}\left(\mathrm{H}_{\mathrm{B}_{\gamma i}}^{U}, \min _{j=i}^{\left|P_{\gamma}\right|} \mathrm{H}_{\mathrm{B}_{\gamma j}}^{U}\right)
\end{array}\right),
\end{aligned}
$$


(3) When $p=1$ and $q=0$, we can obtain

$$
\begin{aligned}
& \operatorname{IT} F_{P H M}{ }^{1,0}\left(\mathrm{~B}_{1}, \mathrm{~B}_{2}, \cdots, \mathrm{B}_{n}\right) \\
& =\left[\begin{array}{c}
\frac{1}{\kappa}\left(\sum_{\gamma=1}^{\kappa}\left(\frac{2}{\left|P_{\gamma}\right|\left(\left|P_{\gamma}\right|+1\right)} \sum_{i=1}^{\left|P_{\gamma}\right|}\left(\left|P_{\gamma}\right|+1-i\right) \beta_{\gamma i 1}^{L}\right)\right), \frac{1}{\kappa}\left(\sum_{\gamma=1}^{\kappa}\left(\frac{2}{\left|P_{\gamma}\right|\left(\left|P_{\gamma}\right|+1\right)} \sum_{i=1}^{\left|P_{\gamma}\right|}\left(\left|P_{\gamma}\right|+1-i\right) \beta_{\gamma i 2}^{L}\right)\right), \\
\frac{1}{\kappa}\left(\sum_{\gamma=1}^{\kappa}\left(\frac{2}{\left|P_{\gamma}\right|\left(\left|P_{\gamma}\right|+1\right)} \sum_{i=1}^{\left|P_{\gamma}\right|}\left(\left|P_{\gamma}\right|+1-i\right) \beta_{\gamma i 3}^{L}\right)\right), \frac{1}{\kappa}\left(\sum_{\gamma=1}^{\kappa}\left(\frac{2}{\left|P_{\gamma}\right|\left(\left|P_{\gamma}\right|+1\right)} \sum_{i=1}^{\left|P_{\gamma}\right|}\left(\left|P_{\gamma}\right|+1-i\right) \beta_{\gamma i 4}^{L}\right)\right) ; \min _{\gamma=1}^{\kappa} \min _{i=1}^{\left|P_{\gamma}\right|}\left(\mathrm{H}_{B_{\gamma i}}^{L} \underset{\substack{\left|P_{\gamma}\right| \\
j=i}}{L} \mathrm{H}_{\mathrm{B}_{\gamma j}}^{L}\right)
\end{array}\right),
\end{aligned}
$$

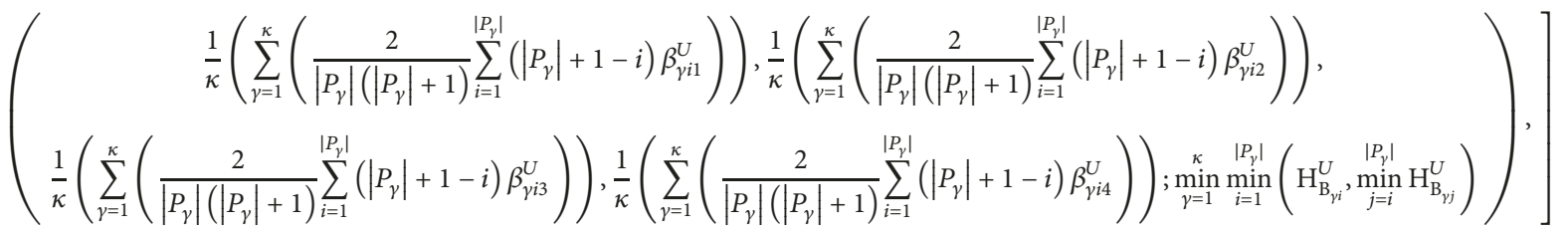

(4) When $p=0$ and $q=1$, we can obtain

$$
\begin{aligned}
& \operatorname{IT} 2 F P H M^{0,1}\left(\mathrm{~B}_{1}, \mathrm{~B}_{2}, \cdots, \mathrm{B}_{n}\right) \\
& =\left[\begin{array}{c}
\frac{1}{\kappa}\left(\sum_{\gamma=1}^{\kappa}\left(\frac{2}{\left|P_{\gamma}\right|\left(\left|P_{\gamma}\right|+1\right)} \sum_{i=1}^{\left|P_{\gamma}\right|} i \beta_{\gamma i 1}^{L}\right)\right), \frac{1}{\kappa}\left(\sum_{\gamma=1}^{\kappa}\left(\frac{2}{\left|P_{\gamma}\right|\left(\left|P_{\gamma}\right|+1\right)} \sum_{i=1}^{\left|P_{\gamma}\right|} i \beta_{\gamma i 2}^{L}\right)\right), \frac{1}{\kappa}\left(\sum_{\gamma=1}^{\kappa}\left(\frac{2}{\left|P_{\gamma}\right|\left(\left|P_{\gamma}\right|+1\right)} \sum_{i=1}^{\left|P_{\gamma}\right|} i \beta_{\gamma i 3}^{L}\right)\right), \\
\frac{1}{\kappa}\left(\sum_{\gamma=1}^{\kappa}\left(\frac{2}{\left|P_{\gamma}\right|\left(\left|P_{\gamma}\right|+1\right)} \sum_{i=1}^{\left|P_{\gamma}\right|} i \beta_{\gamma i 4}^{L}\right)\right) ; \min _{\gamma=1}^{\kappa} \min _{i=1}^{\left|P_{\gamma}\right|}\left(\mathrm{H}_{\mathrm{B}_{\gamma i}}^{L}, \min _{j=i}^{\left|P_{\gamma}\right|} \mathrm{H}_{\mathrm{B}_{\gamma j}}^{L}\right)
\end{array}\right), \\
& \left(\begin{array}{c}
\frac{1}{\kappa}\left(\sum_{\gamma=1}^{\kappa}\left(\frac{2}{\left|P_{\gamma}\right|\left(\left|P_{\gamma}\right|+1\right)} \sum_{i=1}^{\left|P_{\gamma}\right|} i \beta_{\gamma i 1}^{U}\right)\right), \frac{1}{\kappa}\left(\sum_{\gamma=1}^{\kappa}\left(\frac{2}{\left|P_{\gamma}\right|\left(\left|P_{\gamma}\right|+1\right)} \sum_{i=1}^{\left|P_{\gamma}\right|} i \beta_{\gamma i 2}^{U}\right)\right), \frac{1}{\kappa}\left(\sum_{\gamma=1}^{\kappa}\left(\frac{2}{\left|P_{\gamma}\right|\left(\left|P_{\gamma}\right|+1\right)} \sum_{i=1}^{\left|P_{\gamma}\right|} i \beta_{\gamma i 3}^{U}\right)\right), \\
\frac{1}{\kappa}\left(\sum_{\gamma=1}^{\kappa}\left(\frac{2}{\left|P_{\gamma}\right|\left(\left|P_{\gamma}\right|+1\right)} \sum_{i=1}^{\left|P_{\gamma}\right|} i \beta_{\gamma i 4}^{U}\right)\right) ; \min _{\gamma=1}^{\kappa} \min _{i=1}^{\left|P_{\gamma}\right|}\left(\mathrm{H}_{\mathrm{B}_{\gamma i}}^{U} \underset{j=i}{\left|P_{\gamma}\right|} \min _{j=i}^{U}\right)
\end{array}\right),
\end{aligned}
$$

(5) When $p=1$ and $q=1$, we can obtain

$$
\begin{aligned}
& \operatorname{IT} 2 F P H M^{1,1}\left(\mathrm{~B}_{1}, \mathrm{~B}_{2}, \cdots, \mathrm{B}_{n}\right)
\end{aligned}
$$

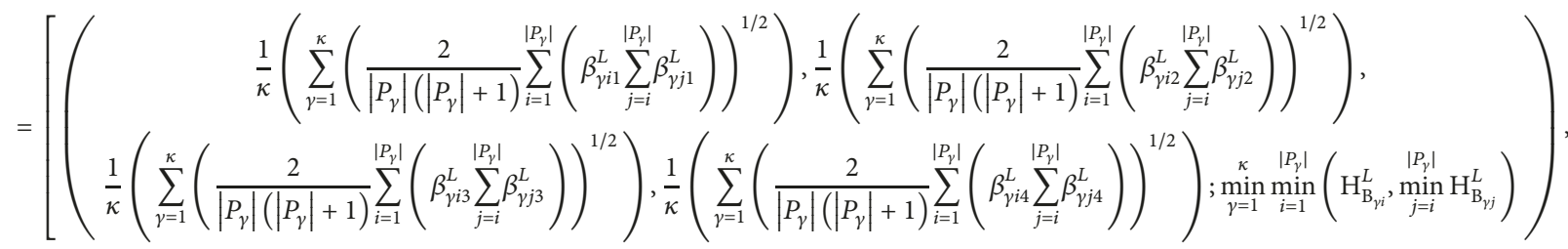

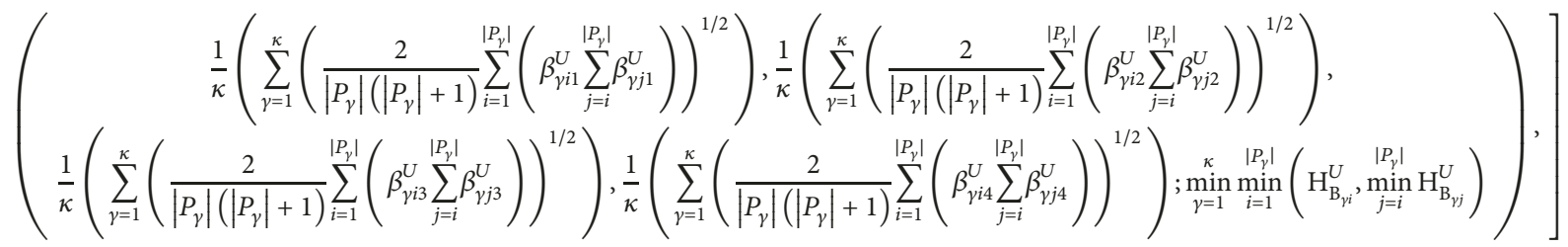


The drawback of the proposed IT2FPHM operator is that it does not address the weights of attributes. Nevertheless, in most practical DM environments, the weights of attributes will significantly influence the final DM orders. Therefore, we now we will develop the interval type- 2 fuzzy weighted PBM (IT2FWPBM) operator to overcome this drawback.

Definition 15. Let $\mathrm{B}=\left\{\mathrm{B}_{i} \mid i=1,2, \cdots n\right\}$ be a collection of IT2FSs, which is divided into $\kappa$ clusters $P_{1}, P_{2}, \cdots, P_{\kappa}$, where $\mathrm{B}_{i}=\left[\left(\beta_{i 1}^{L}, \beta_{i 2}^{L}, \beta_{i 3}^{L}, \beta_{i 4}^{L} ; \mathrm{H}_{\mathrm{B}_{i}}^{L}\right),\left(\beta_{i 1}^{U}, \beta_{i 2}^{U}, \beta_{i 3}^{U}, \beta_{i 4}^{U} ; \mathrm{H}_{\mathrm{B}_{i}}^{U}\right)\right]$, $P_{\gamma}=\left\{\mathrm{B}_{\gamma 1}, \mathrm{~B}_{\gamma 2}, \cdots, \mathrm{B}_{\gamma\left|P_{\gamma}\right|}\right\}\left(\gamma=1,2, \cdots,\left|P_{\gamma}\right|\right), \bigcup_{\gamma=1}^{\kappa} P_{\gamma}=\mathrm{B}$, $\sum_{\gamma=1}^{\kappa}\left|P_{\gamma}\right|=n$, and $\left|P_{\gamma}\right|$ reflects the cardinality of $\mathrm{P}_{\gamma}$. $\omega=\left\{\omega_{i} \mid i=1,2, \cdots, n\right\}$ is a set of weights of $B_{1}, B_{2}, \cdots$, and $\mathrm{B}_{n}, \omega_{i} \in[0,1](i=1,2, \cdots, n)$ and $\sum_{i=1}^{n} \omega_{i}=1$. For any $p, q>0$, the IT2FWPHM operator of $\mathrm{B}_{1}, \mathrm{~B}_{2}, \cdots$, and $\mathrm{B}_{n}$ can be defined as follows:

$$
\operatorname{IT} 2 F W P H M^{p, q}\left(\mathrm{~B}_{1}, \mathrm{~B}_{2}, \cdots, \mathrm{B}_{n}\right)
$$

$$
\begin{aligned}
& =\frac{1}{\kappa}\left(\sum _ { \gamma = 1 } ^ { \kappa } \left(\frac{2}{\left|P_{\gamma}\right|\left(\left|P_{\gamma}\right|+1\right)}\right.\right. \\
& \left.\left.\cdot \sum_{i=1}^{\left|P_{\gamma}\right|\left|P_{\gamma=i}\right|}\left(\omega_{\gamma i} \mathrm{~B}_{\gamma i}\right)^{p} \otimes\left(\omega_{\gamma j} \mathrm{~B}_{\gamma j}\right)^{q}\right)^{1 /(p+q)}\right)
\end{aligned}
$$

Theorem 16. Let $\mathrm{B}=\left\{\mathrm{B}_{i} \mid i=1,2, \cdots n\right\}$ be a collection of IT2FSs, which is divided into $\kappa$ clusters $P_{1}, P_{2}, \cdots, P_{\kappa}$, where $\mathrm{B}_{i}=\left[\left(\beta_{i 1}^{L}, \beta_{i 2}^{L}, \beta_{i 3}^{L}, \beta_{i 4}^{L} ; \mathrm{H}_{\mathrm{B}_{i}}^{L}\right),\left(\beta_{i 1}^{U}, \beta_{i 2}^{U}, \beta_{i 3}^{U}, \beta_{i 4}^{U} ; \mathrm{H}_{\mathrm{B}_{i}}^{U}\right)\right]$, $P_{\gamma}=\left\{\mathrm{B}_{\gamma 1}, \mathrm{~B}_{\gamma 2}, \cdots, \mathrm{B}_{\gamma\left|P_{\gamma}\right|}\right\}\left(\gamma=1,2, \cdots,\left|P_{\gamma}\right|\right), \bigcup_{\gamma=1}^{k} P_{\gamma}=\mathrm{B}$, $\sum_{\gamma=1}^{\kappa}\left|P_{\gamma}\right|=n$, and $\left|P_{\gamma}\right|$ reflects the cardinality of $P_{\gamma}$. $\omega=$ $\left\{\omega_{i} \mid i=1,2, \cdots, n\right\}$ is a set of weights of $\mathrm{B}_{1}, \mathrm{~B}_{2}, \cdots$, and $\mathrm{B}_{n}, \omega_{i} \in[0,1](i=1,2, \cdots, n)$ and $\sum_{i=1}^{n} \omega_{i}=1$. For any $p, q>0$, the aggregated value of the IT2FWPHM operator is still an IT2FS, and

$$
\begin{aligned}
& \operatorname{IT2FPHM}^{p, q}\left(\mathrm{~B}_{1}, \mathrm{~B}_{2}, \cdots, \mathrm{B}_{n}\right)
\end{aligned}
$$

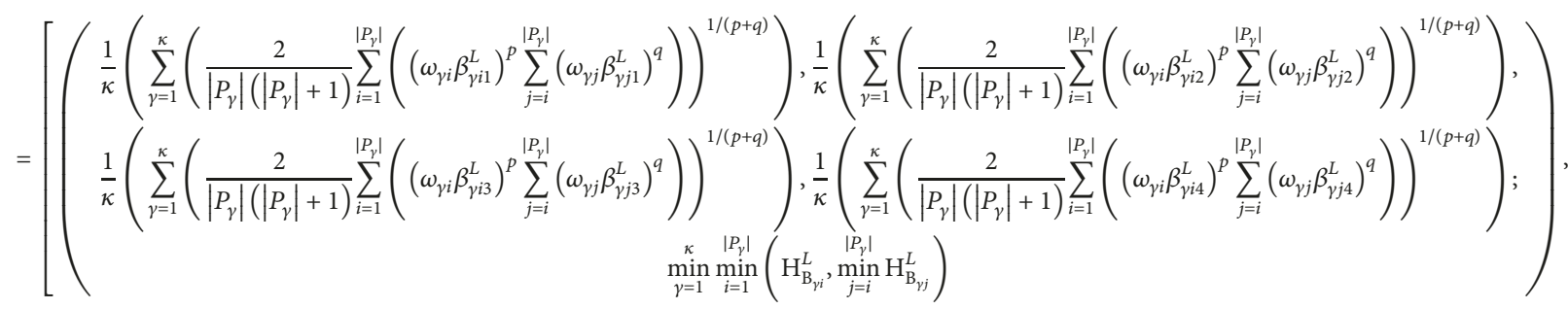

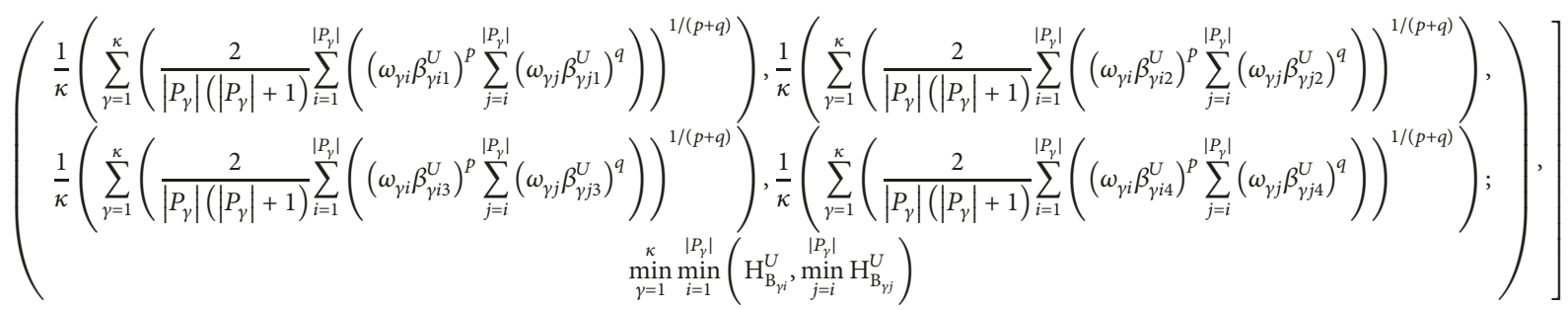

Proof. Based on (7), (11), and (13), we can acquire

$$
\left.\left.\left(\omega_{\gamma i} \beta_{\gamma i 3}^{U}\right)^{p},\left(\omega_{\gamma i} \beta_{\gamma i 4}^{U}\right)^{p} ; \mathrm{H}_{\mathrm{B}_{i}}^{U}\right)\right]
$$

$$
\begin{gathered}
\left(\omega_{\gamma i} \mathrm{~B}_{\gamma i}\right)^{p}=\left[\left(\left(\omega_{\gamma i} \beta_{\gamma i 1}^{L}\right)^{p},\left(\omega_{\gamma i} \beta_{\gamma i 2}^{L}\right)^{p},\left(\omega_{\gamma i} \beta_{\gamma i 3}^{L}\right)^{p},\right.\right. \\
\left.\left(\omega_{\gamma i} \beta_{\gamma i 4}^{L}\right)^{p} ; \mathrm{H}_{\mathrm{B}_{i}}^{L}\right),\left(\left(\omega_{\gamma i} \beta_{\gamma i 1}^{U}\right)^{p},\left(\omega_{\gamma i} \beta_{\gamma i 2}^{U}\right)^{p},\right.
\end{gathered}
$$

and

$$
\begin{aligned}
& \left(\omega_{\gamma i} \mathrm{~B}_{\gamma i}^{L}\right)^{p} \sum_{j=i}^{\left|P_{\gamma}\right|}\left(\mathrm{B}_{\gamma j}^{U}\right)^{q} \\
& =\left[\begin{array}{c}
\left(\omega_{\gamma i} \beta_{\gamma i 1}^{L}\right)^{p} \sum_{j=i}^{\left|P_{\gamma}\right|}\left(\omega_{\gamma j} \beta_{\gamma j 1}^{L}\right)^{q},\left(\omega_{\gamma i} \beta_{\gamma i 2}^{L}\right)^{p} \sum_{j=i}^{\left|P_{\gamma}\right|}\left(\omega_{\gamma j} \beta_{\gamma j 2}^{L}\right)^{q},\left(\omega_{\gamma i} \beta_{\gamma i 3}^{L}\right)^{p} \sum_{j=i}^{\left|P_{\gamma}\right|}\left(\omega_{\gamma j} \beta_{\gamma j 3}^{L}\right)^{q},\left(\omega_{\gamma i} \beta_{\gamma i 4}^{L}\right)^{p} \sum_{j=i}^{\left|P_{\gamma}\right|}\left(\omega_{\gamma j} \beta_{\gamma j 4}^{L}\right)^{q} ; \\
\min \left(\mathrm{H}_{\mathrm{B}_{\gamma i}}^{L}, \min _{j=i}^{\left|P_{\gamma}\right|} \mathrm{H}_{\mathrm{B}_{\gamma j}}^{L}\right)
\end{array}\right),
\end{aligned}
$$




$$
\left.\left(\begin{array}{c}
\left(\omega_{\gamma i} \beta_{\gamma i 1}^{U}\right)^{p} \sum_{j=i}^{\left|P_{\gamma}\right|}\left(\omega_{\gamma j} \beta_{\gamma j 1}^{U}\right)^{q},\left(\omega_{\gamma i} \beta_{\gamma i 2}^{U}\right)^{p} \sum_{j=i}^{\left|P_{\gamma}\right|}\left(\omega_{\gamma j} \beta_{\gamma j 2}^{U}\right)^{q},\left(\omega_{\gamma i} \beta_{\gamma i 3}^{U}\right)^{p} \sum_{j=i}^{\left|P_{\gamma}\right|}\left(\omega_{\gamma j} \beta_{\gamma j 3}^{U}\right)^{q},\left(\omega_{\gamma i} \beta_{\gamma i 4}^{U}\right)^{p} \sum_{j=i}^{\left|P_{\gamma}\right|}\left(\omega_{\gamma j} \beta_{\gamma j 4}^{U}\right)^{q} ; \\
\min \left(\mathrm{H}_{\mathrm{B}_{\gamma i}}^{U}, \min _{j=i}^{\left|P_{\gamma}\right|} \mathrm{H}_{\mathrm{B}_{\gamma j}}^{U}\right)
\end{array}\right)\right]
$$

Then,

$$
\begin{aligned}
& \frac{2}{\left|P_{\gamma}\right|\left(\left|P_{\gamma}\right|+1\right)} \sum_{i=1}^{\left|P_{\gamma}\right|\left|P_{\gamma}\right|} \sum_{j=i}\left(\omega_{\gamma i} \mathrm{~B}_{\gamma i}\right)^{p} \otimes\left(\omega_{\gamma j} \mathrm{~B}_{\gamma j}\right)^{q}
\end{aligned}
$$

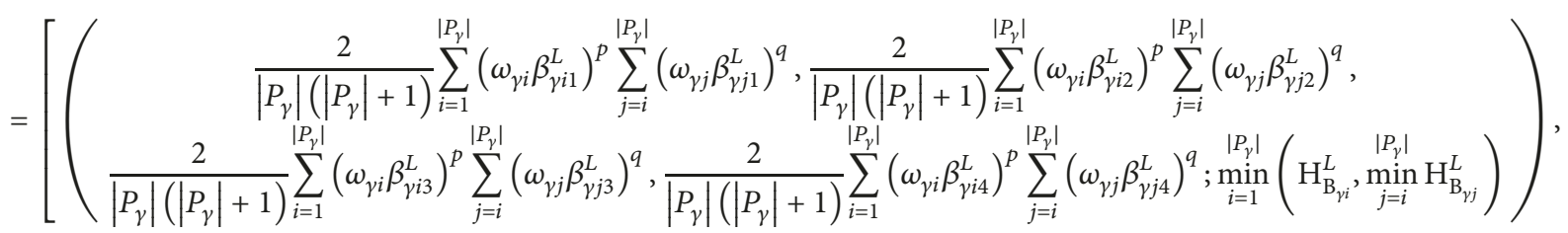

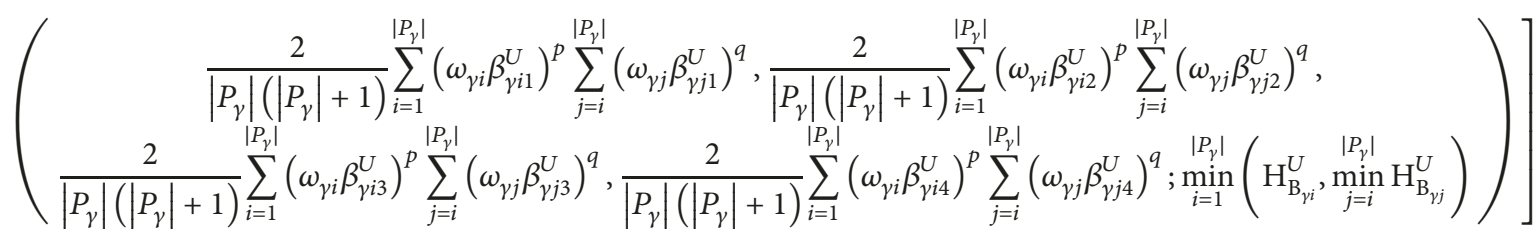

Finally, we can obtain

$$
\begin{aligned}
& \frac{1}{\kappa}\left(\sum_{\gamma=1}^{\kappa}\left(\frac{2}{\left|P_{\gamma}\right|\left(\left|P_{\gamma}\right|+1\right)} \sum_{i=1}^{\left|P_{\gamma}\right|\left|P_{\gamma}\right|}\left(\omega_{j i} \mathrm{~B}_{\gamma i}\right)^{p} \otimes\left(\omega_{\gamma j} \mathrm{~B}_{\gamma j}\right)^{q}\right)^{1 /(p+q)}\right)
\end{aligned}
$$

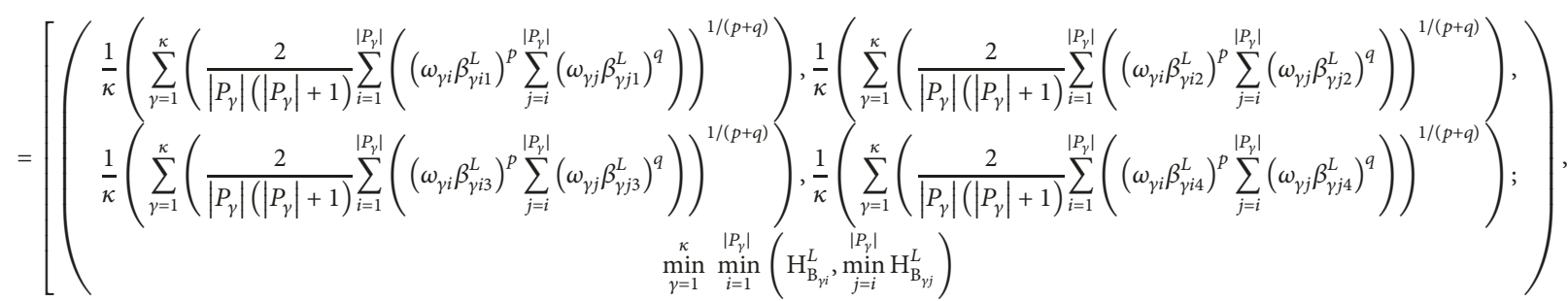

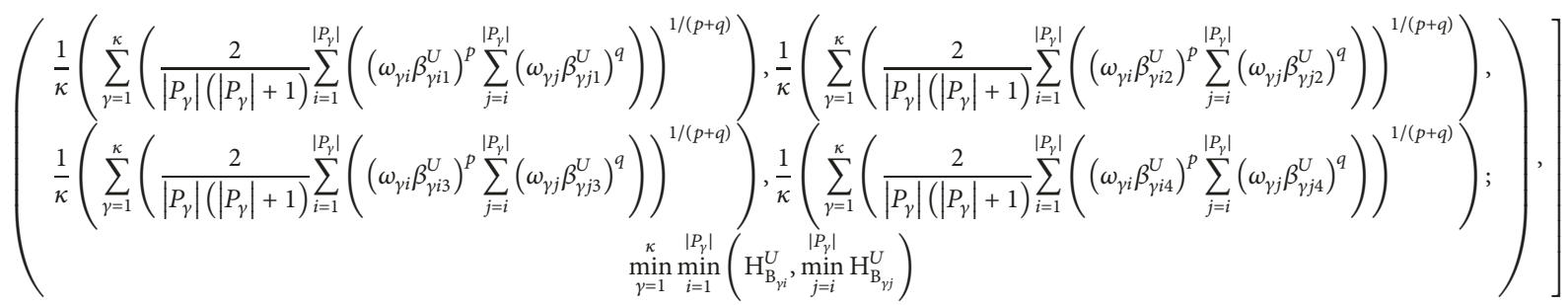

Theorem 17 (idempotency). Let $\mathrm{B}=\left\{\mathrm{B}_{i} \mid i=1,2, \cdots n\right\}$ be a collection of IT2FSs. If all $\mathrm{A}_{i}(i=1,2, \cdots n)$ are equal, that is, $\mathrm{B}_{i}=\mathrm{B}=\left[\left(\beta_{1}^{L}, \beta_{2}^{L}, \beta_{3}^{L}, \beta_{4}^{L} ; \mathrm{H}_{\mathrm{B}}^{L}\right),\left(\beta_{1}^{U}, \beta_{2}^{U}, \beta_{3}^{U}, \beta_{4}^{U} ; \mathrm{H}_{\mathrm{B}}^{U}\right)\right]$ $(i=1,2, \cdots, n)$, then $\omega=\left\{\omega_{i} \mid i=1,2, \cdots, n\right\}$ is a set of weights of $\mathrm{B}_{1}, \mathrm{~B}_{2}, \cdots$, and $\mathrm{B}_{n}, \omega_{i} \in[0,1](i=1,2, \cdots, n)$ and $\sum_{i=1}^{n} \omega_{i}=1$. Then,

$$
\operatorname{IT2FWPHM} M^{a, b}\left(\mathrm{~B}_{1}, \mathrm{~B}_{2}, \cdots, \mathrm{B}_{n}\right)=\mathrm{B} .
$$


TABLE 1: Linguistic terms and their corresponding IT2FSs [35, 37].

\begin{tabular}{lcc}
\hline LTs & LTs & IT2FSs \\
\hline Very bad (VB) & Extremely weak (EW) & {$[(0,0,0,0.1 ; 1),(0,0,0,0.05 ; 0.9)]$} \\
Quite bad (QB) & Very weak (VW) & {$[(0,0.1,0.1,0.3 ; 1),(0.05,0.1,0.1,0.2 ; 0.9)]$} \\
Bad (B) & Weak (W) & {$[(0.1,0.3,0.3,0.5 ; 1),(0.2,0.3,0.3,0.4 ; 0.9)]$} \\
Medium (M) & Medium (M) & {$[(0.3,0.5,0.5,0.7 ; 1),(0.4,0.5,0.5,0.6 ; 0.9)]$} \\
Good (G) & Strong (S) & {$[(0.5,0.7,0.7,0.9 ; 1),(0.6,0.7,0.7,0.8 ; 0.9)]$} \\
Quite Good (QG) & Very strong (VS) & {$[(0.7,0.9,0.9,1 ; 1),(0.8,0.9,0.9,0.95 ; 0.9)]$} \\
Very good (VG) & Extremely strong (ES) & {$[(0.9,1,1,1 ; 1),(0.95,1,1,1 ; 0.9)]$} \\
\hline
\end{tabular}

Theorem 18 (commutativity). Let $\mathrm{B}=\left\{\mathrm{B}_{i} \mid i=1,2, \cdots n\right\}$ and $\mathrm{B}^{\prime}=\left\{\mathrm{B}_{i}^{\prime} \mid i=1,2, \cdots n\right\}$ be two collections of IT2FSs. If $\mathrm{B}_{i}{ }^{\prime}=\left[\left(\beta_{1}^{L^{\prime}}, \beta_{2}^{L^{\prime}}, \beta_{3}^{L^{\prime}}, \beta_{4}^{L^{\prime}} ; \mathrm{H}_{\mathrm{B}}^{L^{\prime}}\right),\left(\beta_{1}^{U^{\prime}}, \beta_{2}^{U^{\prime}}, \beta_{3}^{U^{\prime}}, \beta_{4}^{U^{\prime}} ; \mathrm{H}_{\mathrm{B}}^{U^{\prime}}\right)\right](i=$ $1,2, \cdots, n)$ is any permutation of $\mathrm{B}_{i}=\left[\left(\beta_{1}^{L}, \beta_{2}^{L}, \beta_{3}^{L}\right.\right.$, $\left.\left.\beta_{4}^{L} ; \mathrm{H}_{\mathrm{B}}^{L}\right),\left(\beta_{1}^{U}, \beta_{2}^{U}, \beta_{3}^{U}, \beta_{4}^{U} ; \mathrm{H}_{\mathrm{B}}^{U}\right)\right](i=1,2, \cdots, n), \omega=\left\{\omega_{i} \mid\right.$ $i=1,2, \cdots, n\}$ is a set of weights of $\mathrm{B}_{1}, \mathrm{~B}_{2}, \cdots$, and $\mathrm{B}_{n}, \omega_{i} \in$ $[0,1](i=1,2, \cdots, n)$ and $\sum_{i=1}^{n} \omega_{i}=1$. Then,

$$
\begin{aligned}
& \operatorname{IT} 2 \operatorname{FWPHM}^{p, q}\left(\mathrm{~B}_{1}, \mathrm{~B}_{2}, \cdots, \mathrm{B}_{n}\right) \\
& \quad=I T 2 F W P H M^{p, q}\left(\mathrm{~B}_{1}{ }^{\prime}, \mathrm{B}_{2}{ }^{\prime}, \cdots, \mathrm{B}_{n}{ }^{\prime}\right) .
\end{aligned}
$$

Since the proofs of Theorems 17 and 18 are similar to those of Theorems 13 and 14, respectively, they are omitted here.

\section{A Novel Green Supplier Selection Method Combining QFD with IT2FWPHM Operator}

In this section, we develop an MCGDM method by combining the classical QFD model with the presented IT2FWPHM operator in the interval type-2 fuzzy environment. Suppose that customers and experts give their preferences in linguistic terms and then they are converted into corresponding IT2FSs. The procedures of the developed method are shown as follows.

Suppose that $\mathrm{A}=\left\{\mathrm{A}_{i} \mid i=1,2, \cdots, r\right\}$ is a set of alternatives, $C=\left\{C_{j} \mid j=1,2, \cdots, s\right\}$ is a set of CRs, $T=\left\{T_{f} \mid f=1,2, \cdots, t\right\}$ is a set of TAs, and $Z=\left\{Z_{\tau} \mid\right.$ $\tau=1,2, \cdots, m\}$ is a set of DMs. Of these, $\widetilde{Z}_{1}=\left\{\widetilde{Z}_{\xi} \mid \xi=\right.$ $\left.1,2, \cdots, m_{1}\right\}$ is a set of customers, $\widetilde{Z}_{2}=\left\{\widetilde{Z}_{\sigma} \mid \sigma=1,2, \cdots\right.$, $\left.m_{2}\right\}$ is a set of experts, $\widetilde{Z}_{1} \cup \widetilde{Z}_{2}=Z$, and $m_{1}+m_{2}=m$. The weight of $Z_{\xi}$ is $\omega_{\xi}$, which satisfies $0 \leq \omega_{\xi} \leq 1$ and $\sum_{\xi=1}^{m_{1}} \omega_{\xi}=1$. The weight of $Z_{\sigma}$ is $\omega_{\sigma}$, which satisfies $0 \leq \omega_{\sigma} \leq 1$ and $\sum_{\sigma=1}^{m_{2}} \omega_{\sigma}=1$.

Let $E^{\xi}=\left(e_{i j}\right)^{\xi}(i=1,2, \cdots, r ; j=1,2, \cdots, s)$ be the decision matrix (DMT) given by the $\xi$ th customer, where $e_{i j}$ is the $\mathrm{EV}$ of $\mathrm{A}_{i}$ with respect to $C_{j}$. The weight of attribute $\omega_{j}(j=1,2, \cdots, s)$ satisfies $0 \leq \omega_{j} \leq 1$ and $\sum_{j=1}^{s} \omega_{j}=$ 1. Let $G^{\sigma}=\left(g_{j f}\right)^{\sigma}(j=1,2, \cdots, s ; f=1,2, \cdots, t)$ be the DMT given by the $\sigma$ th expert, where $g_{j f}$ is the EVs of the relationships between the CRs and TAs. The presented method is applied to deal with the LSP selection (LSPS) problems in which all attributes (TAs) are partitioned into several clusters. In these problems, the attributes (TAs) in the same clusters are interrelated, while the attributes in different clusters are independent. For example, the set of TAs includes the distribution speed $\left(\mathrm{T}_{1}\right)$, quality stability $\left(\mathrm{T}_{2}\right)$, encasement $\left(\mathrm{T}_{3}\right)$, professional cold storage and transport equipment $\left(\mathrm{T}_{4}\right)$, flexible time of receipt $\left(\mathrm{T}_{5}\right)$, service-oriented attitude $\left(\mathrm{T}_{6}\right)$, and feedback speed $\left(\mathrm{T}_{7}\right)$. Based on the relationships between the different attributes, we can divide these seven attributes into the following three partitions: $\mathrm{P}_{1}=\left\{\mathrm{T}_{1}, \mathrm{~T}_{5}\right\}, \mathrm{P}_{2}=$ $\left\{\mathrm{T}_{2}, \mathrm{~T}_{3}, \mathrm{~T}_{4}\right\}$, and $\mathrm{P}_{3}=\left\{\mathrm{T}_{6}, \mathrm{~T}_{7}\right\}$. Because the higher the technical level of professional cold storage and transport equipment, the stronger the quality stability, similarly, the greater the service consciousness of a service-oriented business, the faster the feedback speed. so $T_{1}$ is related with $T_{5}$ and $T_{1}$ is not related with $\mathrm{T}_{2}, \mathrm{~T}_{3}, \mathrm{~T}_{4}, \mathrm{~T}_{6}$, and $\mathrm{T}_{7}$.

Step 1. Identify the CRs, the TAs that are associated with the CRs and the advanced product or service design alternatives. Then, the customers $\left(\widetilde{Z}_{1}\right)$ give the EVs of the importance of the CRs by using the provided LTs (see Table 1 ). In addition, the experts $\left(\widetilde{Z}_{2}\right)$ give the EVs of the importance of the relationships between CRs and TAs by using the provided LTs.

Step 2. Normalize DMT $E^{\xi}=\left(e_{i j}\right)^{\xi}(i=1,2, \cdots, r ; j=1,2$, $\cdots, s)$ and $G^{\sigma}=\left(g_{j f}\right)^{\sigma}(j=1,2, \cdots, s ; f=1,2, \cdots, t)$. If the attributes are different types, we should normalize the DMT. Normalize the original DMT using the following formula.

$$
\begin{gathered}
\tilde{e}_{i j}= \begin{cases}e_{i j} & \text { for benefit criteria } \\
\left(e_{i j}\right)^{c} & \text { for cost criteria }\end{cases} \\
\tilde{g}_{j f}= \begin{cases}g_{j f} & \text { for benefit criteria } \\
\left(g_{j f}\right)^{c} & \text { for cost criteria }\end{cases}
\end{gathered}
$$

where $\left(e_{i j}\right)^{c}$ and $\left(g_{j f}\right)^{c}$ are the complements of $e_{i j}$ and $g_{j f}$ listed in Table 2 , respectively. $\widetilde{e}_{i j}$ and $\widetilde{g}_{j f}$ are the normalized forms of $e_{i j}$ and $g_{j f}$, respectively.

Step 3. Convert the normalized LTs into the corresponding IT2FSs based on Table 1, which can be, respectively, denoted as follows:

$$
\begin{aligned}
\widetilde{E}^{\xi}= & \left(\widetilde{e}_{i j}\right)^{\xi} \\
= & {\left[\begin{array}{c}
\left(\left(\beta_{i j 1}^{L}\right)^{\xi},\left(\beta_{i j 2}^{L}\right)^{\xi},\left(\beta_{i j 3}^{L}\right)^{\xi},\left(\beta_{i j 4}^{L}\right)^{\xi} ;\left(\mathrm{H}_{i j}^{L}\right)^{\xi}\right), \\
\left(\left(\beta_{i j 1}^{U}\right)^{\xi},\left(\beta_{i j 2}^{U}\right)^{\xi},\left(\beta_{i j 3}^{U}\right)^{\xi},\left(\beta_{i j 4}^{U}\right)^{\xi} ;\left(\mathrm{H}_{i j}^{U}\right)^{\xi}\right)
\end{array}\right] } \\
& \left(i=1,2, \cdots, r ; j=1,2, \cdots, s ; \xi=1,2, \cdots, m_{1}\right)
\end{aligned}
$$


TABLE 2: The complementary relations $[35,37]$.

\begin{tabular}{llllllll}
\hline LT & VB/EW & QB/VW & B/W & M & G/S & QG/VS \\
\hline$(\text { LT })^{\mathrm{C}}$ & VG/ES & QG/VS & G/S & M & B/W & QB/VW & VB/EW \\
\hline
\end{tabular}

$$
\begin{aligned}
G^{\sigma}= & \left(\tilde{g}_{j f}\right)^{\sigma} \\
= & {\left[\begin{array}{c}
\left(\left(\delta_{j f 1}^{L}\right)^{\sigma},\left(\delta_{j f 2}^{L}\right)^{\sigma},\left(\delta_{j f 3}^{L}\right)^{\sigma},\left(\delta_{j f 4}^{L}\right)^{\sigma} ;\left(\mathrm{H}_{j l}^{L}\right)^{\sigma}\right), \\
\left(\left(\delta_{j f 1}^{U}\right)^{\sigma},\left(\delta_{j f 2}^{U}\right)^{\sigma},\left(\delta_{j f 3}^{U}\right)^{\sigma},\left(\delta_{j f 4}^{U}\right)^{\sigma} ;\left(\mathrm{H}_{j l}^{U}\right)^{\sigma}\right)
\end{array}\right] } \\
& \left(j=1,2, \cdots, s ; f=1,2, \cdots, t ; \sigma=1,2, \cdots, m_{2}\right)
\end{aligned}
$$

Step 4. Aggregate the EVs of all individuals into overall EVs using the WA operator. If the weights of the customers and experts are not given, we can assume that $\omega_{\xi}=1 / m_{1}(\xi=$ $\left.1,2, \cdots, m_{1}\right), \omega_{\sigma}=1 / m_{2}\left(\sigma=1,2, \cdots, m_{2}\right)$. Then, we have

$$
\begin{aligned}
& \widetilde{E}=\widetilde{e}_{i j}=I T 2 F S-W A\left(\left(\widetilde{e}_{i j}\right)^{1},\left(\widetilde{e}_{i j}\right)^{2}, \cdots,\left(\widetilde{e}_{i j}\right)^{m_{1}}\right)=\sum_{\xi=1}^{m_{1}} \omega_{\xi}\left(\widetilde{e}_{i j}\right)^{\xi} \\
& =\left[\begin{array}{c}
\left(\sum_{\xi=1}^{m_{1}} \omega_{\xi}\left(\beta_{i j 1}^{L}\right)^{\xi}, \sum_{\xi=1}^{m_{1}} \omega_{\xi}\left(\beta_{i j 2}^{L}\right)^{\xi}, \sum_{\xi=1}^{m_{1}} \omega_{\xi}\left(\beta_{i j 3}^{L}\right)^{\xi}, \sum_{\xi=1}^{m_{1}} \omega_{\xi}\left(\beta_{i j 4}^{L}\right)^{\xi} ; \min _{\xi=1}^{m_{1}}\left(\left(\mathrm{H}_{i j}^{L}\right)^{\xi}\right)\right), \\
\left(\sum_{\xi=1}^{m_{1}} \omega_{\xi}\left(\beta_{i j 1}^{U}\right)^{\xi}, \sum_{\xi=1}^{m_{1}} \omega_{\xi}\left(\beta_{i j 2}^{U}\right)^{\xi}, \sum_{\xi=1}^{m_{1}} \omega_{\xi}\left(\beta_{i j 3}^{U}\right)^{\xi}, \sum_{\xi=1}^{m_{1}} \omega_{\xi}\left(\beta_{i j 4}^{U}\right)^{\xi} ; \min _{\xi=1}^{m_{1}}\left(\left(\mathrm{H}_{i j}^{U}\right)^{\xi}\right)\right.
\end{array}\right] \\
& \left(i=1,2, \cdots, \mathrm{r} ; j=1,2, \cdots, \mathrm{s} ; \xi=1,2, \cdots, m_{1}\right)
\end{aligned}
$$

and

$$
\begin{aligned}
& \widetilde{G}=\widetilde{g}_{j f}=I T 2 F S-W A\left(\left(\tilde{g}_{j f}\right)^{1},\left(\tilde{g}_{j f}\right)^{2}, \cdots,\left(\tilde{g}_{j f}\right)^{m_{2}}\right)=\sum_{\sigma=1}^{m_{2}} \omega_{\sigma}\left(\tilde{g}_{j f}\right)^{\sigma} \\
& =\left[\begin{array}{c}
\left(\sum_{\sigma=1}^{m_{2}} \omega_{\sigma}\left(\delta_{j f 1}^{L}\right)^{\sigma}, \sum_{\sigma=1}^{m_{2}} \omega_{\sigma}\left(\delta_{j f 2}^{L}\right)^{\sigma}, \sum_{\sigma=1}^{m_{2}} \omega_{\sigma}\left(\delta_{j f 3}^{L}\right)^{\sigma}, \sum_{\sigma=1}^{m_{2}} \omega_{\sigma}\left(\delta_{j f 4}^{L}\right)^{\sigma} ; \min _{\sigma=1}^{m_{2}}\left(\mathrm{H}_{j f}^{L}\right)^{\sigma}\right), \\
\left(\sum_{\sigma=1}^{m_{2}} \omega_{\sigma}\left(\delta_{j f 1}^{L}\right)^{\sigma}, \sum_{\sigma=1}^{m_{2}} \omega_{\sigma}\left(\delta_{j f 2}^{L}\right)^{\sigma}, \sum_{\sigma=1}^{m_{2}} \omega_{\sigma}\left(\delta_{j f 3}^{L}\right)^{\sigma}, \sum_{\sigma=1}^{m_{2}} \omega_{\sigma}\left(\delta_{j f 4}^{L}\right)^{\sigma} ; \min _{\sigma=1}^{m_{2}}\left(\mathrm{H}_{j l}^{U}\right)^{\sigma}\right)
\end{array}\right] \\
& \left(j=1,2, \cdots, t ; l=1,2, \cdots, r ; \sigma=1,2, \cdots, m_{2}\right)
\end{aligned}
$$

Step 5. Compute the optimal weights of the CRs. In this method, the weights of the CRs may not be fully known. Generally, the weights for attributes can be acquired by applying some classic methods, such as the maximum deviation method [39]. The noticeable character of this method is that if the EVs of each alternative with respect to the attributes have larger deviations among the diverse alternatives, in such a scenario, this attribute should be assigned a greater weight than the other attributes. In general, we can maximize all deviation values for all the attributes when selecting the weight vector $\omega=\left(\omega_{1}, \omega_{2}, \cdots, \omega_{s}\right)$.

For the developed method in this paper, we can establish a programming model as follows:

$$
\max \Theta(\omega)=\sum_{j=1}^{s} \sum_{i=1}^{r} \Theta_{i j}(\omega)
$$

s.t. $\quad 0 \leq \omega_{j}, \sum_{j=1}^{s} \omega_{j}^{2}=1, j=1,2, \cdots, s$

where $\Theta_{i j}(\omega)=\sum_{\nu=1}^{r}\left|D\left(\widetilde{e}_{i j}, \tilde{\widetilde{1}}\right)-D\left(\widetilde{e}_{\nu j}, \widetilde{\widetilde{1}}\right)\right| \omega_{j}(i=1,2, \cdots$, $r ; j=1,2, \cdots, s)$ is the deviation value (DV) of $\mathrm{A}_{i}$ with respect to all the other alternatives with respect to attribute $C_{j}$, and $\Theta_{j}(\omega)=\sum_{i=1}^{r} \sum_{v=1}^{r}\left|D\left(\widetilde{e}_{i j}, \widetilde{\widetilde{1}}\right)-D\left(\widetilde{e}_{v j}, \widetilde{\widetilde{1}}\right)\right| \omega_{j}$ 
$(j=1,2, \cdots, s)$ is the DV of all alternatives with respect to all other alternatives for attribute $C_{j}$.

To deal with model (47), the Lagrange function is established as follows:

$$
\begin{aligned}
L(\omega, \lambda)= & \sum_{j=1}^{s} \sum_{i=1}^{r} \sum_{v=1}^{r}\left|D\left(\widetilde{e}_{i j}, \widetilde{\widetilde{1}}\right)-D\left(\widetilde{e}_{v j}, \widetilde{\widetilde{1}}\right)\right| \omega_{j} \\
& +\frac{\lambda}{2}\left(\sum_{j=1}^{s} \omega_{j}^{2}-1\right)
\end{aligned}
$$

where $\lambda$ is the Lagrange multiplier.

By taking the derivatives, we can get two equations as follows:

$$
\begin{aligned}
\frac{\partial L}{\partial \omega_{j}} & =\sum_{i=1}^{r} \sum_{\nu=1}^{r}\left|D\left(\widetilde{e}_{i j}, \widetilde{\tilde{1}}\right)-D\left(\widetilde{e}_{\nu j}, \widetilde{\tilde{1}}\right)\right|+\lambda \omega_{j}=0 \\
\frac{\partial L}{\partial \lambda} & =\sum_{j=1}^{s} \omega_{j}^{2}-1=0
\end{aligned}
$$

By solving (49) and (50), we can acquire the following:

$$
\begin{array}{r}
\omega_{j}=\frac{\sum_{i=1}^{r} \sum_{v=1}^{r}\left|D\left(\tilde{e}_{i j}, \tilde{\tilde{1}}\right)-D\left(\tilde{e}_{v j}, \tilde{\tilde{1}}\right)\right|}{\sqrt{\sum_{j=1}^{s}\left(\sum_{i=1}^{r} \sum_{v=1}^{r}\left|D\left(\tilde{e}_{i j}, \tilde{\tilde{1}}\right)-D\left(\widetilde{e}_{v j}, \tilde{1}\right)\right|\right)^{2}}} \\
\quad(j=1,2, \cdots, s)
\end{array}
$$

Through normalization, we get the following:

$$
\begin{array}{r}
\widehat{\omega}_{j}=\frac{\sum_{i=1}^{r} \sum_{v=1}^{r}\left|D\left(\widetilde{e}_{i j}, \tilde{1}\right)-D\left(\widetilde{e}_{v j}, \tilde{1}\right)\right|}{\sum_{j=1}^{s} \sum_{i=1}^{r} \sum_{\rho=1}^{r}\left|D\left(\widetilde{e}_{i j}, \tilde{1}\right)-D\left(\widetilde{e}_{v j}, \tilde{1}\right)\right|} \\
\quad(j=1,2, \cdots, s)
\end{array}
$$

Step 6. Calculate the weights of the TAs. From Section 2.1, we can see that, based on the QFD, the optimal weights of the CRs are converted into the weights with respect to TAs. Therefore, the weights of the TAs can be obtained based on the relationship $\widetilde{g}_{j f}$ and the weights of $C_{j}$.

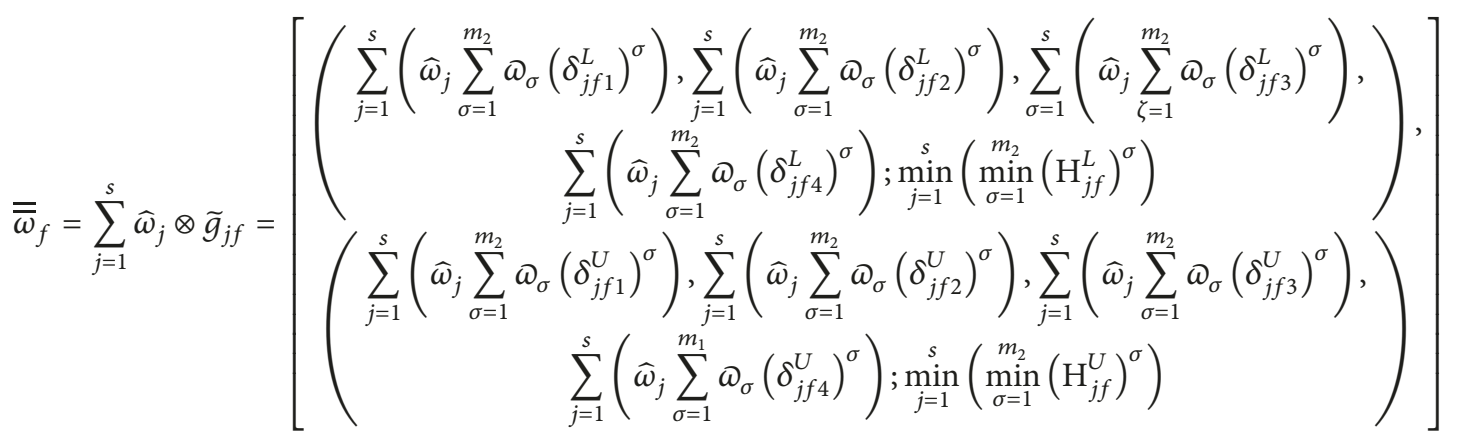

$$
\begin{aligned}
& (j=1,2, \cdots, s ; f=1,2, \cdots, t)
\end{aligned}
$$

To reduce the computational complexity, we can also obtain the following:

$$
w_{f}=D\left(\overline{\bar{\omega}}_{f}-\tilde{\widetilde{1}}\right)
$$

Step 7. Calculate the ranking of the alternatives using the TAs.

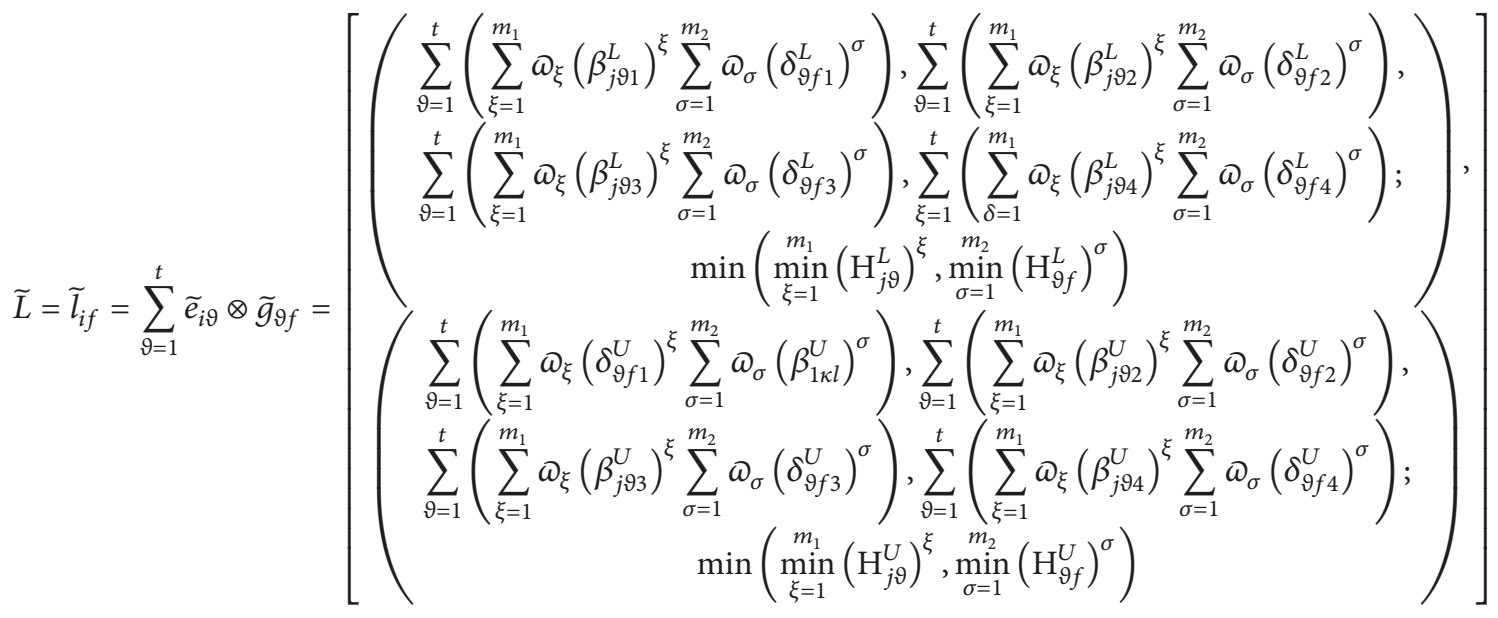

$$
\begin{aligned}
& (i=1,2, \cdots, r ; f=1,2, \cdots, t)
\end{aligned}
$$


Step 8. Apply the proposed IT2FWPHM operator in order to acquire the comprehensive preference value of alternative $\mathrm{A}_{i}(i=1,2, \cdots, r)$ with respect to all $T_{f}(f=1,2, \cdots, t)$ (the
TAs $T_{f}(f=1,2, \cdots, t)$ in the same clusters are interrelated, while the attributes in different clusters are independent) and acquire

$$
\begin{aligned}
& R_{t}=I T 2 F W P H M^{p, q}\left(\widetilde{l}_{t 1}, \tilde{l}_{t 2}, \cdots, \tilde{l}_{t f}\right) \\
& {\left[\left(\begin{array}{c}
\frac{1}{\kappa}\left(\sum_{\gamma=1}^{\kappa}\left(\frac{2}{\left|P_{\gamma}\right|\left(\left|P_{\gamma}\right|+1\right)} \sum_{i=1}^{\left|P_{\gamma}\right|}\left(\left(\omega_{\gamma i} \beta_{\gamma i 1}^{L}\right)^{p} \sum_{j=i}^{\left|P_{\gamma}\right|}\left(\omega_{\gamma j} \beta_{\gamma j 1}^{L}\right)^{q}\right)\right)^{1 /(p+q)}\right), \frac{1}{\kappa}\left(\sum_{\gamma=1}^{\kappa}\left(\frac{2}{\left|P_{\gamma}\right|\left(\left|P_{\gamma}\right|+1\right)} \sum_{i=1}^{\left|P_{\gamma}\right|}\left(\left(\omega_{\gamma i} \beta_{\gamma i 2}^{L}\right)^{p} \sum_{j=i}^{\left|P_{\gamma}\right|}\left(\omega_{\gamma j} \beta_{\gamma j 2}^{L}\right)^{q}\right)\right)\right. \\
\frac{1}{\kappa}\left(\sum_{\gamma=1}^{\kappa}\left(\frac{2}{\left|P_{\gamma}\right|\left(\left|P_{\gamma}\right|+1\right)} \sum_{i=1}^{\left|P_{\gamma}\right|}\left(\left(\omega_{\gamma i} \beta_{\gamma i 3}^{L}\right)^{p} \sum_{j=i}^{\left|P_{\gamma}\right|}\left(\omega_{\gamma j} \beta_{\gamma j 3}^{L}\right)^{q}\right)\right)^{1 /(p+q)}\right), \frac{1}{\kappa}\left(\sum_{\gamma=1}^{\kappa}\left(\frac{2}{\left|P_{\gamma}\right|\left(\left|P_{\gamma}\right|+1\right)} \sum_{i=1}^{\left|P_{\gamma}\right|}\left(\left(\omega_{\gamma i} \beta_{\gamma i 4}^{L}\right)^{p} \sum_{j=i}^{\left|P_{\gamma}\right|}\left(\omega_{\gamma j} \beta_{\gamma j 4}^{L}\right)^{q}\right)\right)\right. \\
\min _{\gamma=1}^{\kappa} \min _{i=1}^{\left|P_{\gamma}\right|}\left(\mathrm{H}_{\mathrm{B}_{\gamma i}}^{L} \min _{j=i}^{\left|P_{\gamma}\right|} \mathrm{H}_{\mathrm{B}_{\gamma j}}^{L}\right)
\end{array}\right) ;\right.}
\end{aligned}
$$

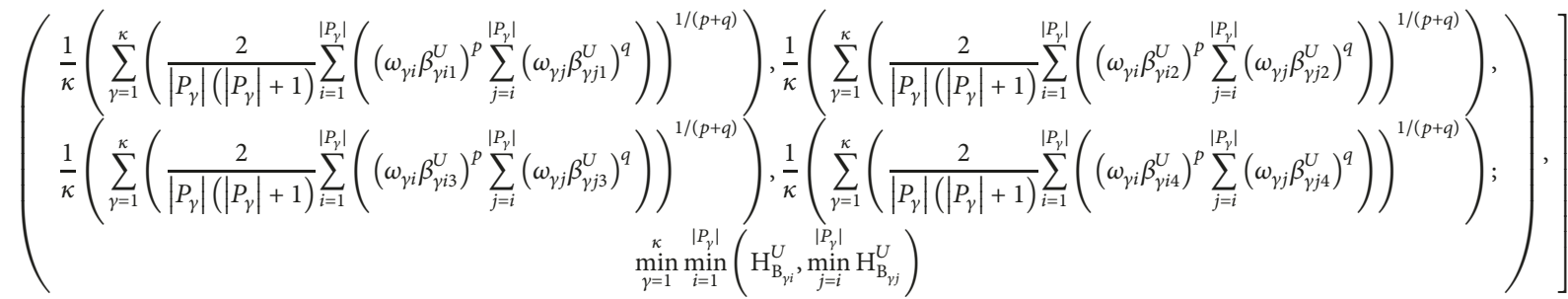

Step 9. Calculate the $D\left(R_{i}-1\right)(i=1,2, \cdots, r)$ based on (16).

Step 10. Rank the alternatives $\mathrm{A}_{i}(i=1,2, \cdots, r)$.

\section{The Application Case: The Fresh E-Business Logistics Service Supplier Selection}

In this section, we will verify the effectiveness and practicability of the proposed novel MCGDM method by applying a fresh E-business logistics service provider (FELSP) selection case and demonstrate its advantages by comparing it with other existing different methods.

5.1. Case Description. Fresh E-business (FE) refers to the direct sale of fresh products on the Internet by means of electronic commerce, such as fresh fruits, vegetables, fresh meat, and so on. With income and consumption chances, the demand for fresh products has increased greatly. Now, the FE market of China is developing rapidly with an average annual growth rate of more than $50 \%$. In such a setting, a large number of FE platform enterprises appear in the market. Fresh products have high requirements for retaining freshness and preventing spoilage, which require timely logistics and safety. Therefore, the logistics service's ability is the key to reducing costs, improving efficiency, and ensuring good quality and stability.

To achieve long-term development and sustained competitiveness, FE platform enterprises must select a suitable FELSP. However, under the rapid development of the FE market, a variety of problems endlessly emerge, especially in logistics services, which seriously affects consumers' consumption of fresh products. Therefore, it is very important and meaningful to evaluate the FELSP from the perspective of customer satisfaction. Obviously, the FELSP is an MCGDM problem. We will apply the presented novel MCGDM method in this paper in order to support a well-known FE platform enterprise and select the best FELSP from some FELSPs.

There are four FELSPs $\mathrm{A}=\left\{\mathrm{A}_{i} \mid i=1,2, \cdots, 4\right\}$ that are provided by the strategic management department of this FE platform enterprise. Through interviews with customers who often buy fresh products from FE platforms, five main CRs emerge, including freshness $\left(C_{1}\right)$, quality $\left(C_{2}\right)$, reliability $\left(C_{3}\right)$, safety $\left(C_{4}\right)$, and empathy $\left(C_{5}\right)$, which are determined to be the biggest concerns for the consumption of fresh products. Furthermore, the set of TAs, which are the FELSP attributes, include the distribution speed $\left(\mathrm{T}_{1}\right)$, quality stability $\left(\mathrm{T}_{2}\right)$, encasement $\left(\mathrm{T}_{3}\right)$, professional cold storage and transport equipment $\left(\mathrm{T}_{4}\right)$, flexible time of receipt $\left(\mathrm{T}_{5}\right)$, serviceoriented attitude $\left(\mathrm{T}_{6}\right)$, and feedback speed $\left(\mathrm{T}_{7}\right)$. Based on the relationships between the different attributes, we can divide these seven attributes into the following three partitions: $\mathrm{P}_{1}=$ $\left\{\mathrm{T}_{1}, \mathrm{~T}_{5}\right\}, \mathrm{P}_{2}=\left\{\mathrm{T}_{2}, \mathrm{~T}_{3}, \mathrm{~T}_{4}\right\}$, and $\mathrm{P}_{3}=\left\{\mathrm{T}_{6}, \mathrm{~T}_{7}\right\}$. Then, forty customers $\widetilde{Z}_{1}=\left\{\widetilde{Z}_{\xi} \mid \xi=1,2, \cdots, 40\right\}$ with the same weights are surveyed and interviewed with respect to the importance of the CRs by using provided LTs (see Table 1). In addition, four experts $\left(\widetilde{Z}_{2}=\left\{\widetilde{Z}_{\sigma} \mid \sigma=1,2, \cdots, 4\right\}\right)$, including a third-party logistics service designer, a logistics department manager, a supply chain management professional, and a logistics management professor with the same weights, are asked to provide EVs for the relationships between the CRs and TAs by using the provided LTs (see Table 1). Two specially designed questionnaires are conducted in order to gather the customers' and experts' EVs, respectively.

\subsection{The Decision Steps Using the Proposed Method Based on IT2FWPHM Operator.}

Step 1. Identify the CRs, the TAs associating with the CRs, and the advanced alternatives. Then, the customers $\left(\widetilde{Z}_{1}\right)$ 
TABLE 3: The decision matrix $E^{1}$ provided by the $1^{\text {st }}$ customer.

\begin{tabular}{lccccc}
\hline & $C_{1}$ & $C_{2}$ & $C_{3}$ & $C_{4}$ & \\
\hline$E_{1}$ & $\mathrm{~B}$ & $\mathrm{M}$ & $\mathrm{QG}$ & $\mathrm{G}$ & $\mathrm{C}$ \\
$E_{2}$ & $\mathrm{QG}$ & $\mathrm{QB}$ & $\mathrm{G}$ & $\mathrm{VB}$ & $\mathrm{M}$ \\
$E_{3}$ & $\mathrm{QB}$ & $\mathrm{G}$ & $\mathrm{M}$ & $\mathrm{G}$ & $\mathrm{VG}$ \\
$E_{4}$ & $\mathrm{~B}$ & $\mathrm{VG}$ & $\mathrm{B}$ & $\mathrm{QB}$ & $\mathrm{M}$ \\
\hline
\end{tabular}

TABLE 4: The relation matrix $G^{1}$ provided by the $1^{\text {st }}$ expert.

\begin{tabular}{lcccccc}
\hline & $\mathrm{T}_{1}$ & $\mathrm{~T}_{2}$ & $\mathrm{~T}_{3}$ & $\mathrm{~T}_{4}$ & $\mathrm{~T}_{5}$ & \\
\hline$C_{1}$ & $\mathrm{VS}$ & $\mathrm{ES}$ & $\mathrm{M}$ & $\mathrm{VW}$ & $\mathrm{T}$ & $\mathrm{TS}$ \\
$C_{2}$ & $\mathrm{VS}$ & $\mathrm{S}$ & $\mathrm{VS}$ & $\mathrm{SS}$ & $\mathrm{S}$ \\
$C_{3}$ & $\mathrm{M}$ & $\mathrm{M}$ & $\mathrm{M}$ & $\mathrm{M}$ & $\mathrm{S}$ & $\mathrm{EW}$ \\
$C_{4}$ & $\mathrm{~S}$ & $\mathrm{VS}$ & $\mathrm{ES}$ & $\mathrm{SW}$ & $\mathrm{S}$ \\
$C_{5}$ & $\mathrm{~W}$ & $\mathrm{VS}$ & $\mathrm{W}$ & & $\mathrm{VW}$ \\
\hline
\end{tabular}

provide the EVs of the importance of CRs. Furthermore, the experts $\left(\widetilde{Z}_{2}\right)$ provide the EVs of the relationships between the CRs and TAs. The EVs are listed in Tables 3 and 4 (due to space constraints, we give only one customer's and one expert's EV).

Step 2. Normalize DMT $E^{\xi}=\left(e_{i j}\right)^{\xi}(i=1,2, \cdots, 4 ; j=$ $1,2, \cdots, 5)$ and $G^{\sigma}=\left(g_{j f}\right)^{\sigma}(j=1,2, \cdots, 5 ; f=1,2, \cdots, 7)$.

Step 3. Convert the LTs into the corresponding IT2FSs.

Step 4. Aggregate the EVs of all individuals into overall EVs in the IT2FSs using the WA operator. If the weights of the customers and experts are not provided, we can suppose that $\omega_{\xi}=1 / 40(\xi=1,2, \cdots, 40), \omega_{\sigma}=1 / 4(\sigma=1,2, \cdots, 4)$.

$$
\begin{aligned}
& \widetilde{E}=\widetilde{e}_{i j}=I T 2 F S-W A\left(\left(\widetilde{e}_{i j}\right)^{1},\left(\widetilde{e}_{i j}\right)^{2}, \cdots,\left(\widetilde{e}_{i j}\right)^{40}\right) \\
&=\sum_{\xi=1}^{40} \omega_{\xi}\left(\widetilde{e}_{i j}\right)^{\xi} \quad(i=1,2, \cdots, 4 ; j=1,2, \cdots, 5) \\
& \widetilde{e}_{11}=[(0.38,0.47,0.47,0.66 ; 1), \\
&(0.39,0.47,0.47,0.59 ; 0.9)], \\
& \widetilde{e}_{12}=[(0.41,0.65,0.65,0.69 ; 1), \\
&\quad(0.44,0.65,0.65,0.64 ; 0.9)], \\
& \widetilde{e}_{13}=[(0.35,0.47,0.47,0.67 ; 1), \\
&\quad(0.39,0.47,0.47,0.61 ; 0.9)], \\
& \widetilde{e}_{14}=[(0.43,0.57,0.57,0.71 ; 1), \\
&\quad(0.43,0.57,0.57,0.65 ; 0.9)], \\
& \widetilde{e}_{15}=[(0.41,0.51,0.51,0.76 ; 1), \\
&\quad(0.38,0.51,0.51,0.64 ; 0.9)], \\
& \widetilde{e}_{21}=[(0.43,0.57,0.57,0.74 ; 1),
\end{aligned}
$$

$(0.52,0.57,0.57,0.71 ; 0.9)]$

$\widetilde{e}_{22}=[(0.39,0.57,0.57,0.83 ; 1)$,

$(0.47,0.57,0.57,0.79 ; 0.9)]$,

$\tilde{e}_{23}=[(0.39,0.65,0.65,0.71 ; 1)$,

$(0.52,0.65,0.65,0.64 ; 0.9)]$,

$\widetilde{e}_{24}=[(0.41,0.49,0.49,0.68 ; 1)$,

$(0.53,0.49,0.49,0.66 ; 0.9)]$,

$\tilde{e}_{25}=[(0.41,0.55,0.55,0.72 ; 1)$,

$(0.46,0.55,0.55,0.66 ; 0.9)]$,

$\widetilde{e}_{31}=[(0.33,0.54,0.54,0.72 ; 1)$,

$(0.40,0.54,0.54,0.67 ; 0.9)]$,

$\tilde{e}_{32}=[(0.39,0.58,0.58,0.69 ; 1)$,

$(0.47,0.58,0.58,064 . ; 0.9)]$,

$\widetilde{e}_{33}=[(0.29,0.49,0.49,0.65 ; 1)$,

$(0.33,0.49,0.49,0.59 ; 0.9)]$,

$\widetilde{e}_{34}=[(0.37,0.56,0.56,0.66 ; 1)$,

$(0.43,0.56,0.56,0.71 ; 0.9)]$,

$\widetilde{e}_{35}=[(0.49,0.67,0.67,0.81 ; 1)$,

$(0.56,0.67,0.67,0.76 ; 0.9)]$,

$\widetilde{e}_{41}=[(0.36,0.54,0.54,0.71 ; 1)$,

$(0.44,0.54,0.54,0.67 ; 0.9)]$,

$\widetilde{e}_{42}=[(0.48,0.71,0.71,0.82 ; 1)$,

$(0.55,0.71,0.71,0.79 ; 0.9)]$,

$\widetilde{e}_{43}=[(0.39,0.58,0.58,0.71 ; 1)$, 
$(0.47,0.58,0.58,0.67 ; 0.9)]$,

$\widetilde{e}_{44}=[(0.41,0.63,0.63,0.78 ; 1)$,

$(0.47,0.63,0.63,0.70 ; 0.9)]$,

$\tilde{e}_{45}=[(0.38,0.48,0.48,0.68 ; 1)$,

$(0.42,0.48,0.48,0.61 ; 0.9)]$.

$$
\begin{aligned}
\widetilde{G} & =\widetilde{g}_{j f}=I T 2 F S-W A\left(\left(\widetilde{g}_{j f}\right)^{1},\left(\widetilde{g}_{j f}\right)^{2}, \cdots,\left(\widetilde{g}_{j f}\right)^{4}\right) \\
& =\sum_{\sigma=1}^{4} \omega_{\sigma}\left(\widetilde{g}_{j f}\right)^{\sigma} \quad(j=1,2, \cdots, 5 ; f=1,2, \cdots, 7)
\end{aligned}
$$

$\widetilde{g}_{11}=[(0.51,0.68,0.68,0.81 ; 1)$,

$(0.59,0.68,0.68,0.75 ; 0.9)]$,

$\widetilde{g}_{12}=[(0.32,0.46,0.46,0.61 ; 1)$,

$(0.37,0.46,0.46,0.53 ; 0.9)]$,

$\widetilde{g}_{13}=[(0.39,0.61,0.61,0.76 ; 1)$,

$(0.48,0.61,0.61,0.67 ; 0.9)]$,

$\widetilde{g}_{14}=[(0.33,0.48,0.48,0.65 ; 1)$,

$(0.40,0.48,0.48,0.56 ; 0.9)]$,

$\widetilde{g}_{15}=[(0.35,0.55,0.55,0.75 ; 1)$,

$(0.45,0.55,0.55,0.65 ; 0.9)]$,

$\widetilde{g}_{16}=[(0.44,0.64,0.64,0.81 ; 1)$,

$(0.53,0.64,0.64,0.73 ; 0.9)]$,

$\widetilde{g}_{17}=[(0.35,0.59,0.59,0.75 ; 1)$,

$(0.41,0.59,0.59,0.68 ; 0.9)]$,

$\widetilde{g}_{21}=[(0.76,0.81,0.81,0.98 ; 1)$,

$(0.79,0.81,0.81,0.92 ; 0.9)]$,

$\widetilde{g}_{22}=[(0.31,0.48,0.48,0.57 ; 1)$,

$(0.39,0.48,0.48,0.51 ; 0.9)]$,

$\tilde{g}_{23}=[(0.71,0.81,0.81,0.95 ; 1)$,

$(0.79,0.81,0.81,0.91 ; 0.9)]$,

$\widetilde{g}_{24}=[(0.44,0.56,0.56,0.66 ; 1)$,

$(0.50,0.56,0.56,0.61 ; 0.9)]$,

$\widetilde{g}_{25}=[(0.25,0.41,0.41,0.55 ; 1)$,

$(0.35,0.41,0.41,0.55 ; 0.9)]$,

$\widetilde{g}_{26}=[(0.44,0.59,0.59,0.74 ; 1)$,

$(0.51,0.59,0.59,0.66 ; 0.9)]$,

$\tilde{g}_{27}=[(0.24,0.48,0.48,0.64 ; 1)$,
$(0.31,0.48,0.48,0.56 ; 0.9)]$,

$\widetilde{g}_{31}=[(0.24,0.44,0.44,0.64 ; 1)$,

$(0.34,0.44,0.44,0.54 ; 0.9)]$,

$\widetilde{g}_{32}=[(0.21,0.31,0.31,0.53 ; 1)$,

$(0.30,0.31,0.31,0.45 ; 0.9)]$,

$\widetilde{g}_{33}=[(0.06,0.13,0.13,0.29 ; 1)$,

$(0.09,0.13,0.13,0.19 ; 0.9)]$,

$\tilde{g}_{34}=[(0.31,0.56,0.56,0.65 ; 1)$,

$(0.41,0.56,0.56,0.60 ; 0.9)]$,

$\widetilde{g}_{35}=[(0.29,0.43,0.43,0.59 ; 1)$,

$(0.36,0.43,0.43,0.51 ; 0.9)]$,

$\widetilde{g}_{36}=[(0.20,0.33,0.33,0.48 ; 1)$,

$(0.26,0.33,0.33,0.40 ; 0.9)]$,

$\widetilde{g}_{37}=[(0.22,0.36,0.36,0.51 ; 1)$,

$(0.27,0.36,0.36,0.43 ; 0.9)]$,

$\widetilde{g}_{41}=[(0.33,0.51,0.51,0.63 ; 1)$,

$(0.40,0.51,0.51,0.55 ; 0.9)]$,

$\tilde{g}_{42}=[(0.34,0.41,0.41,0.64 ; 1)$,

$(0.38,0.41,0.41,0.55 ; 0.9)]$,

$\widetilde{g}_{43}=[(0.45,0.65,0.65,0.86 ; 1)$,

$(0.55,0.65,0.65,0.77 ; 0.9)]$,

$\widetilde{g}_{44}=[(0.39,0.58,0.58,0.74 ; 1)$,

$(0.48,0.58,0.58,0.64 ; 0.9)]$,

$\widetilde{g}_{45}=[(0.31,0.45,0.45,0.59 ; 1)$,

$(0.39,0.45,0.45,0.48 ; 0.9)]$,

$\widetilde{g}_{46}=[(0.25,0.41,0.41,0.65 ; 1)$,

$(0.35,0.41,0.41,0.55 ; 0.9)]$,

$\widetilde{g}_{47}=[(0.41,0.66,0.66,0.75 ; 1)$,

$(0.51,0.66,0.66,0.70 ; 0.9)]$,

$\tilde{g}_{51}=[(0.21,0.29,0.29,0.40 ; 1)$,

$(0.26,0.29,0.29,0.36 ; 0.9)]$,

$\widetilde{g}_{52}=[(0.39,0.58,0.58,0.73 ; 1)$,

$(0.48,0.58,0.58,0.65 ; 0.9)]$,

$\tilde{g}_{53}=[(0.45,0.65,0.65,0.75 ; 1)$,

$(0.55,0.65,0.65,0.74 ; 0.9)]$, 


$$
\begin{gathered}
\widetilde{g}_{54}=[(0.41,0.59,0.59,0.74 ; 1), \\
(0.49,0.59,0.59,0.65 ; 0.9)], \\
\widetilde{g}_{55}=[(0.24,0.34,0.34,0.59 ; 1), \\
(0.29,0.34,0.34,0.43 ; 0.9)], \\
\widetilde{g}_{56}=[(0.30,0.41,0.41,0.48 ; 1), \\
(0.34,0.41,0.41,0.43 ; 0.9)], \\
\widetilde{g}_{57}=[(0.38,0.60,0.60,0.75 ; 1), \\
(0.47,0.60,0.60,0.66 ; 0.9)] .
\end{gathered}
$$

Step 5. Compute the optimal weight of the CRs.

Based on (53), we can get $\widehat{\omega}_{j}=(0.24,0.19,0.26,0.13,0.18)$

Step 6. Calculate the weights of the TAs.

Based on the QFD, the optimal weights of the CRs are converted into the respective weights for the TAs. Therefore, the weights of the TAs can be obtained based on the relationship of $\tilde{g}_{j f}$ and the weights of $C_{j} \cdot \overline{\bar{\omega}}_{j}=\widehat{\omega}_{j} \otimes \tilde{g}_{j f}=$ $(0.22,0.11,0.16,0.17,0.13,0.06,0.15)$.

Step 7. Calculate the ranking of the alternatives using the TAs and obtain

$$
\begin{aligned}
& \widetilde{L}=\widetilde{l}_{i f}=\sum_{\vartheta=1}^{t} \tilde{e}_{i \vartheta} \otimes \tilde{g}_{\vartheta f} \\
& (i=1,2, \cdots, 4 ; f=1,2, \cdots, 7) \\
& \widetilde{l}_{11}=[(0.49,0.71,0.71,0.80 ; 1) \text {, } \\
& (0.55,0.71,0.71,0.76 ; 0.9)] \text {, } \\
& \tilde{l}_{12}=[(0.31,0.46,0.46,0.66 ; 1) \text {, } \\
& (0.36,0.46,0.46,0.58 ; 0.9)], \\
& \widetilde{l}_{13}=[(0.39,0.69,0.69,0.83 ; 1) \text {, } \\
& (0.44,0.69,0.69,0.76 ; 0.9)], \\
& \tilde{l}_{14}=[(0.35,0.44,0.44,0.64 ; 1) \text {, } \\
& (0.40,0.44,0.44,0.58 ; 0.9)], \\
& \widetilde{l}_{15}=[(0.32,0.55,0.55,0.74 ; 1) \text {, } \\
& (0.40,0.55,0.55,0.68 ; 0.9)] \text {, } \\
& \tilde{l}_{16}=[(0.57,0.73,0.73,0.92 ; 1) \text {, } \\
& (0.63,0.73,0.73,0.85 ; 0.9)], \\
& \tilde{l}_{17}=[(0.34,0.51,0.51,0.68 ; 1) \text {, } \\
& (0.42,0.51,0.51,0.59 ; 0.9)] \text {, } \\
& \tilde{l}_{21}=[(0.71,0.78,0.78,0.89 ; 1) \text {, }
\end{aligned}
$$

$$
(0.75,0.78,0.78,0.84 ; 0.9)]
$$$$
\widetilde{l}_{22}=[(0.51,0.64,0.64,0.77 ; 1) \text {, }
$$$$
(0.58,0.64,0.64,0.71 ; 0.9)],
$$$$
\tilde{l}_{23}=[(0.58,0.69,0.69,0.79 ; 1) \text {, }
$$$$
(0.62,0.69,0.69,0.73 ; 0.9)] \text {, }
$$$$
\widetilde{l}_{24}=[(0.48,0.56,0.56,0.67 ; 1) \text {, }
$$$$
(0.51,0.56,0.56,0.62 ; 0.9)] \text {, }
$$

$$
\begin{gathered}
\tilde{l}_{25}=[(0.41,0.58,0.58,0.79 ; 1), \\
(0.49,0.58,0.58,0.68 ; 0.9)],
\end{gathered}
$$$$
\tilde{l}_{26}=[(0.28,0.43,0.43,0.58 ; 1) \text {, }
$$$$
(0.34,0.43,0.43,0.49 ; 0.9)] \text {, }
$$

$\tilde{l}_{27}=[(0.49,0.79,0.79,0.93 ; 1)$,

$(0.54,0.79,0.79,0.86 ; 0.9)]$,

$\tilde{l}_{31}=[(0.17,0.38,0.38,0.54 ; 1)$, $(0.26,0.38,0.38,0.46 ; 0.9)]$,

$\tilde{l}_{32}=[(0.19,0.26,0.26,0.41 ; 1)$, $(0.23,0.26,0.26,0.38 ; 0.9)]$, $\widetilde{l}_{33}=[(0.11,0.16,0.16,0.34 ; 1)$, $(0.13,0.16,0.16,0.28 ; 0.9)]$, $\widetilde{l}_{34}=[(0.40,0.51,0.51,0.62 ; 1)$, $(0.44,0.51,0.51,0.58 ; 0.9)]$, $\widetilde{l}_{35}=[(0.31,0.45,0.45,0.56 ; 1)$, $(0.38,0.45,0.45,0.51 ; 0.9)]$, $\tilde{l}_{36}=[(0.31,0.47,0.47,0.61 ; 1)$, $(0.36,0.47,0.47,0.52 ; 0.9)]$, $\widetilde{l}_{37}=[(0.32,0.49,0.49,0.68 ; 1)$, $(0.38,0.49,0.49,0.60 ; 0.9)]$, $\tilde{l}_{41}=[(0.29,0.48,0.48,0.67 ; 1)$, $(0.33,0.48,0.48,0.59 ; 0.9)]$, $\tilde{l}_{42}=[(0.31,0.44,0.44,0.71 ; 1)$, $(0.39,0.44,0.44,0.62 ; 0.9)]$, $\tilde{l}_{43}=[(0.37,0.65,0.65,0.81 ; 1)$, $(0.45,0.65,0.65,0.73 ; 0.9)]$, 


$$
\begin{gathered}
\tilde{l}_{44}=[(0.44,0.61,0.61,0.78 ; 1), \\
(0.52,0.61,0.61,0.69 ; 0.9)], \\
\tilde{l}_{45}=[(0.17,0.26,0.26,0.59 ; 1), \\
(0.21,0.26,0.26,0.52 ; 0.9)], \\
\tilde{l}_{46}=[(0.42,0.60,0.60,0.76 ; 1),
\end{gathered}
$$

$(0.48,0.60,0.60,0.69 ; 0.9)]$,

$\tilde{l}_{47}=[(0.28,0.49,0.49,0.65 ; 1)$,

$(0.37,0.49,0.49,0.57 ; 0.9)]$.

Step 8. Apply the novel proposed IT2FWPHM operator (suppose $p=q=1$ ) in order to obtain

$$
\begin{aligned}
& R_{i}=I T 2 F W P H M^{p, q}\left(\tilde{l}_{i 1}, \widetilde{l}_{i 2}, \cdots, \tilde{l}_{i 7}\right)
\end{aligned}
$$

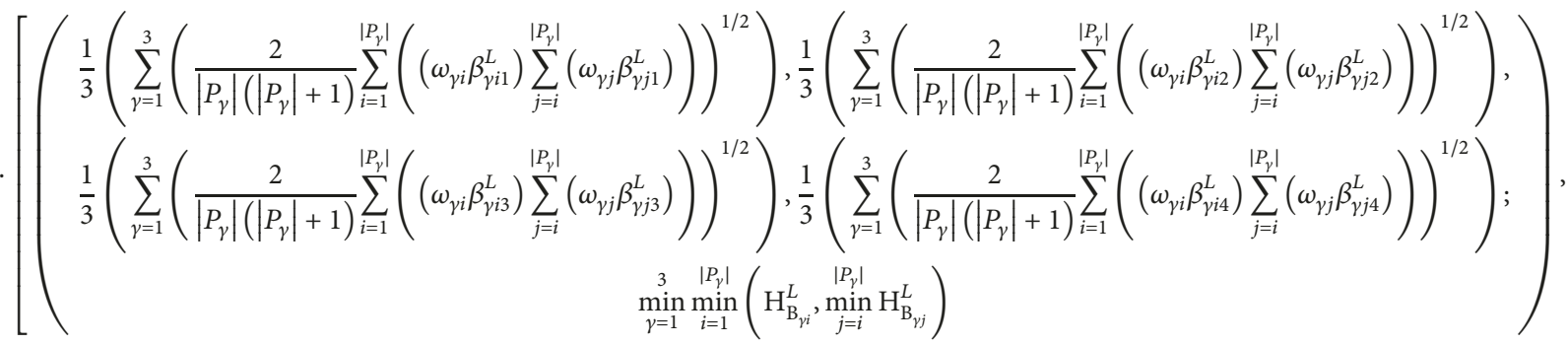

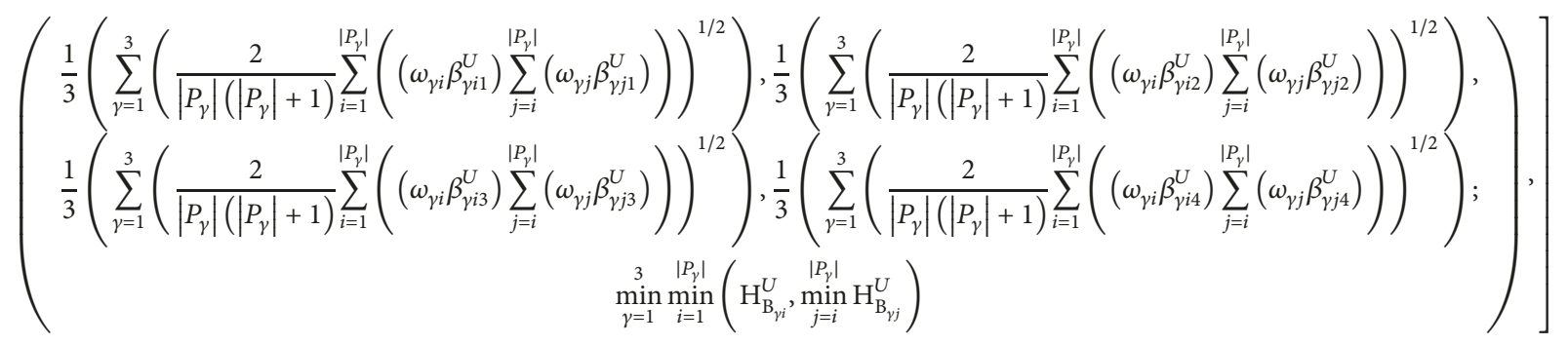

$$
\begin{aligned}
& (i=1,2, \cdots, 4) \\
& R_{2}=[(0.0392,0.0568,0.0568,0.0737 ; 1),(0.0401,0.0568,0.0568,0.0674 ; 0.9)] \text {, } \\
& R_{3}=[(0.0294,0.0385,0.0385,0.0532 ; 1),(0.0308,0.0385,0.0385,0.0497 ; 0.9)] \text {, } \\
& R_{4}=[(0.0452,0.0511,0.0511,0.0690 ; 1),(0.0490,0.0511,0.0511,0.0604 ; 0.9)] .
\end{aligned}
$$

Step 9. Calculate the $D\left(R_{i}-1\right)(i=1,2, \cdots, s)$ based on (16) and obtain

$$
\begin{aligned}
& D\left(R_{1}-\tilde{\widetilde{1}}\right)=0.8984, \\
& D\left(R_{2}-\tilde{\widetilde{1}}\right)=0.9096, \\
& D\left(R_{3}-\tilde{\tilde{1}}\right)=0.9258, \\
& D\left(R_{4}-\tilde{\widetilde{1}}\right)=0.9109 .
\end{aligned}
$$

Step 10. Rank the alternatives $\mathrm{A}_{i}(i=1,2, \cdots, r)$ and obtain

$$
\mathrm{A}_{3}>\mathrm{A}_{4}>\mathrm{A}_{2}>\mathrm{A}_{1} \text {. }
$$

Therefore, the best FELSP is $\mathrm{E}_{3}$.

5.3. An Exploration of the Influence of Parameters $p$ and $q$ on Final Result for This Case. The rankings for the different parameters $p$ and $q$ are shown in Figures 3-6.
The analysis of the selection of suitable values of parameters $p$ and $q$ is conducted. With $\mathrm{A}_{1}$ as an example, the expected values $\left(D\left(R_{1}-\tilde{\tilde{1}}\right)\right)$ acquired applying IT2FWPHM operator with different values of parameters $p$ and $q$. Figure 3 denotes that with the increase of the values of parameters $p$ and $q$, the aggregate values become increasingly bigger. The comparison of Figures 3 and 4 indicates that the expected values in Figure 3 are smaller than those in Figure 4.

From Figures 3-6, we can visually see that the final ranking orders of the above case are different because of the different values of parameters $p$ and $q$ in the developed MCGDM method. For instances, when $p=1$ and $q=7$, the ranking order is $A_{3}>A_{2}>A_{4}>A_{1}$. However, the best FELSP is still $\mathrm{A}_{3}$. As a general rule, the bigger the values of parameters $p$ and $q$, the more complex that they are to calculate, but the more priority that is given to the interrelationships between TAs. Customers and experts can assign the values of parameters $p$ and $q$ based on their preferences in a specific decision-making process $[21,22,27]$. 
TABLE 5: Ranking orders of the different methods for the FELSP selection case in Section 5.1.

\begin{tabular}{lll}
\hline Methods & \multicolumn{1}{c}{ Ranking values } & Ranking orders \\
\hline $\begin{array}{l}\text { Zhang's method [19] based on the GTIT2FWA } \\
\text { operator and GTIT2FHA operator }(\lambda=1)\end{array}$ & $Q_{1}=122.37, Q_{2}=125.91$, & $\mathrm{A}_{3}>\mathrm{A}_{4}>\mathrm{A}_{2}>\mathrm{A}_{1}$ \\
\hline Wang et al.s method [22] & $Q_{3}=131.98, Q_{4}=128.43$. & $\mathrm{A}_{3}>\mathrm{A}_{4}>\mathrm{A}_{2}>\mathrm{A}_{1}$ \\
based on the TIT2FWGHM operator $(p=q=1)$ & $\mathrm{Q}_{1}=0.3108, \mathrm{Q}_{2}=0.3527$, & $\mathrm{Q}_{3}=0.3959, Q_{4}=0.3803$. \\
\hline $\begin{array}{l}\text { the proposed novel method } \\
\text { based on the IT2FWPHM operator }(p=q=1)\end{array}$ & $\mathrm{Q}_{1}=0.8984, Q_{2}=0.9096$, & $\mathrm{A}_{3}>\mathrm{A}_{4}>\mathrm{A}_{1}$ \\
\hline
\end{tabular}

Note. $\mathrm{Q}_{i}$ is the abbreviation of the ranking value of $\mathrm{A}_{i}(i=1,2,3,4)$.

Expected Values for $\mathrm{A}_{1}$ obtained by IT2FWPHM operator

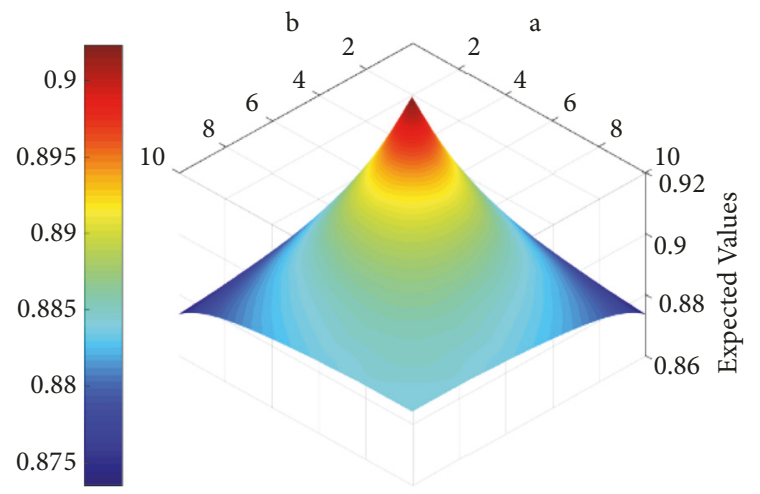

FIGURE 3: $D\left(R_{1}-\tilde{\widetilde{1}}\right)$ of $\mathrm{A}_{1}$ based on IT2FWPHM operator.

Expected Values for $\mathrm{A}_{2}$ obtained by IT2FWPHM operator

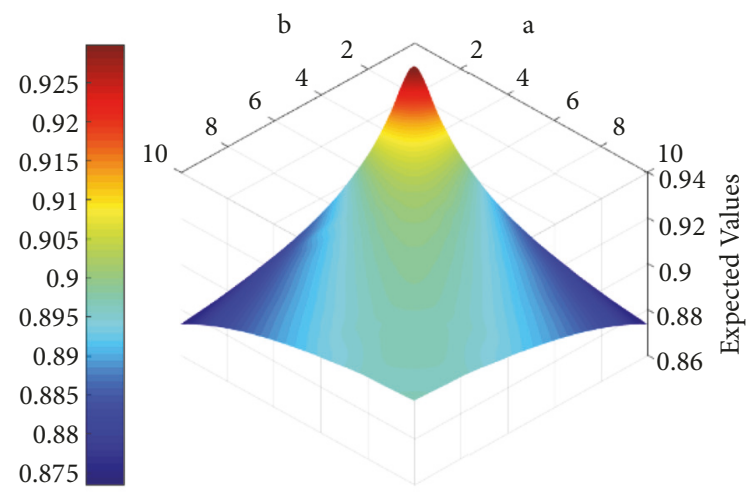

FIGURE 4: $D\left(R_{2}-\tilde{\widetilde{1}}\right)$ of $\mathrm{A}_{2}$ based on IT2FWPHM operator.

When they are optimistic, bigger values can be assigned to parameters $p$ and $q$. Conversely, when they are pessimistic, smaller values can be assigned to parameters $p$ and $q$. As a rule, we can set $p=q=1$, which is intuitive and easy to compute and considers the interrelationship of the individual arguments.

5.4. The Verification of the Validity and Rationality. In the following, in order to verify the validity and rationality of the proposed novel MCGDM method based on the proposed IT2FWPHM operator in this paper, we apply Zhang's method
Expected Values for $\mathrm{A}_{3}$ obtained by IT2FWPHM operator

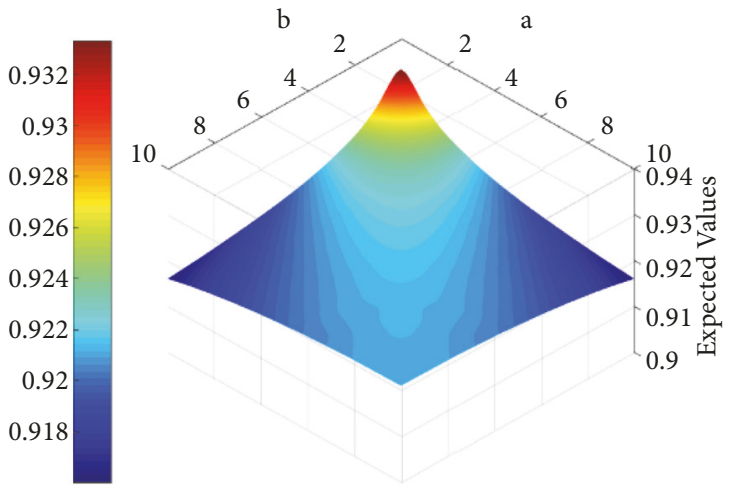

FIgURE 5: $D\left(R_{3}-\tilde{\tilde{1}}\right)$ of $\mathrm{A}_{3}$ based on IT2FWPHM operator.

Expected Values for $\mathrm{A}_{4}$ obtained by IT2FWPHM operator

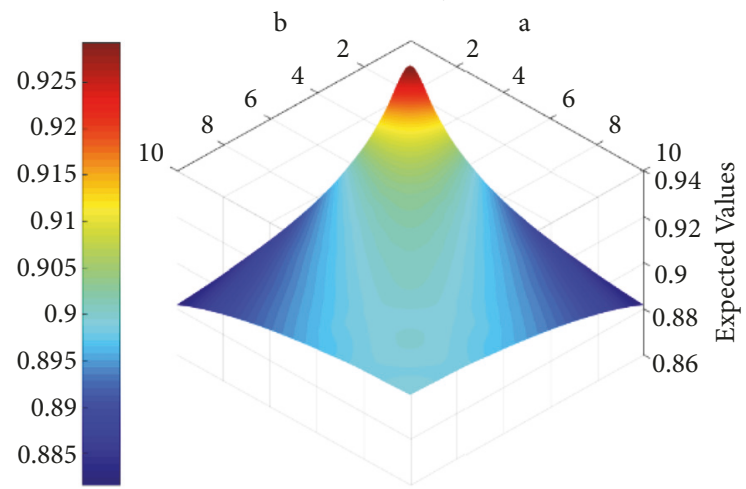

FIgURE 6: $D\left(R_{4}-\tilde{\widetilde{1}}\right)$ of $\mathrm{A}_{4}$ based on IT2FWPHM operator.

[19] based on the GTIT2FWA operator $(\lambda=1)$ and TIT2FHA operator $(\lambda=1)$ and Wang et al.s method [22] based on the TIT2F weighted generalized HM (TIT2FWGHM) operator $(p=q=1)$ in order to address the FELSP selection case in Section 5.1 and perform contrast analysis of the rankings of these three methods. Their ranking orders are listed in Table 5 .

It is worth noting that Zhang's method [19] and Wang et al.s method [22] have no Steps 1-6 like the proposed novel method in Section 4. In other words, they are not combined with the QFD. 
TABLE 6: The decision matrix $\widetilde{L}$ provided by the expert $Z_{1}$.

\begin{tabular}{lcccc}
\hline & $T_{1}$ & $T_{2}$ & $T_{3}$ & \\
\hline$E_{1}$ & G & VG & QG & M \\
$E_{2}$ & VG & B & VG & QG \\
$E_{3}$ & QG & VG & B & VG \\
\hline
\end{tabular}

TABLE 7: The decision matrix $\widetilde{L}$ provided by the expert $Z_{2}$.

\begin{tabular}{lcccc}
\hline & $T_{1}$ & $T_{2}$ & $T_{3}$ & \\
\hline$E_{1}$ & G & VG & VG & \\
$E_{2}$ & QG & QG & QB & M \\
$E_{3}$ & B & QB & QG & VG \\
\hline
\end{tabular}

TABLE 8: The decision matrix $\widetilde{L}$ provided by the expert $Z_{3}$.

\begin{tabular}{lcccc}
\hline & $T_{1}$ & $T_{2}$ & $T_{3}$ & $T_{4}$ \\
\hline$E_{1}$ & QG & VG & B & VG \\
$E_{2}$ & $\mathrm{M}$ & $\mathrm{QG}$ & $\mathrm{VG}$ & $\mathrm{QG}$ \\
$E_{3}$ & $\mathrm{VG}$ & $\mathrm{QB}$ & $\mathrm{G}$ & $\mathrm{G}$ \\
\hline
\end{tabular}

TABLE 9: Rankings of the different methods for Example 1.

\begin{tabular}{lcc}
\hline Methods & Ranking values & Ranking orders \\
\hline $\begin{array}{l}\text { Zhang's method [19] based on the GTIT2FWA } \\
\text { operator and the GTIT2FHA operator }(\lambda=1)\end{array}$ & $Q_{1}=135.96, Q_{2}=126.57$, & $\mathrm{A}_{2}>108.54$. \\
\hline $\begin{array}{l}\text { Wang et al.'s method [22] } \\
\text { based on the TIT2FWGHM operator }(p=q=1)\end{array}$ & $\mathrm{Q}_{1}=0.3916, \mathrm{Q}_{2}=0.3902$, \\
\hline $\begin{array}{l}\text { the presented method } \\
\text { based on the IT2FWPHM operator }(p=1, q=0)\end{array}$ & $\mathrm{Q}_{3}=0.3652$. & $\mathrm{A}_{1}>\mathrm{A}_{2}>\mathrm{A}_{3}$ \\
\hline $\begin{array}{l}\text { the proposed novel method } \\
\text { based on the IT2FWPHM operator }(p=q=1)\end{array}$ & $\mathrm{Q}_{1}=0.8987, Q_{2}=0.8994$, & $\mathrm{A}_{2}>\mathrm{A}_{1}>\mathrm{A}_{3}$ \\
\hline
\end{tabular}

Note. $Q_{i}$ is the abbreviation of the ranking of $\mathrm{A}_{i}(i=1,2,3)$.

From Table 5, we can visually find that the ranking based on the proposed novel method $(p=q=1)$ is the same as Zhang's method [19] and Wang et al.'s method [22], i.e., $\mathrm{A}_{3}>\mathrm{A}_{4}>\mathrm{A}_{2}>\mathrm{A}_{1}$. Clearly, this situation can illustrate the validity and rationality of the proposed novel method.

5.5. Further Comparative Analysis with the Method in [19, 22]. In the above Section 5.4, the validity and rationality of the proposed novel method have been verified by comparing them with those of the two existing methods $[19,22]$. However, due to their identical ranking, it is not powerful enough to demonstrate the delightful advantages (DAs) of the proposed novel method and the limitations of the two existing methods $[19,22]$ in a real case. Therefore, we apply the proposed novel method and other two existing methods $[19,22]$ in order to solve two other numerical examples. Example 1 will demonstrate the DAs of the HM operator of the IT2FSs by comparing them with Zhang's method [19] based on the GTIT2FWA operator and the GTIT2FHA operator and Example 2 will demonstrate the DAs of the proposed novel method based on the IT2FWPHM operator by comparing them with Wang et al.'s method [22] based on the TIT2FWGHM operator.

Example 1 ([19]). There are three candidate suppliers A = $\left\{\mathrm{A}_{i} \mid i=1,2,3\right\}$ that are stipulated by the enterprise's procurement organization. Three experts $\left(Z=\left\{Z_{\tau} \mid \tau=\right.\right.$ $1,2,3\}$ with the same weight are invited to provide EVs of $\mathrm{T}=\left\{\mathrm{T}_{f} \mid f=1,2, \cdots, 4\right\}$ by using the given LTs. The weights of $\mathrm{T}=\left\{\mathrm{T}_{f} \mid f=1,2, \cdots, 4\right\}$ are $\overline{\bar{\omega}}_{j}=(0.28,0.23 .0 .35,0.14)$. The EVs are listed in Tables 6-8. The ranking orders based on the different methods are listed in Table 9.

From Table 9, we realize that the ranking that is obtained by the proposed novel method ( $p=1, q=0$ ) is the same as the one based on Zhang's method [19], i.e., $A_{2}>A_{1}>$ $\mathrm{A}_{3}$, and the ranking that is obtained by the novel proposed method ( $p=q=1$ ) is the same as the one based on Wang et al.'s method [22] $(p=q=1)$, i.e., $\mathrm{A}_{1}>\mathrm{A}_{2}>\mathrm{A}_{3}$. We can 
TABLE 10: Ranking orders of the different methods for Example 2.

\begin{tabular}{llc}
\hline Methods & Ranking values & Ranking orders \\
\hline Wang et al.s method [19] & $\mathrm{Q}_{1}=0.3108, \mathrm{Q}_{2}=0.3749$, & $\mathrm{A}_{3}>\mathrm{A}_{2}>\mathrm{A}_{4}>\mathrm{A}_{1}$ \\
based on the TIT2FWGHM operator & $\mathrm{Q}_{3}=0.3959, \mathrm{Q}_{4}=0.3604$. & $\mathrm{A}_{3}>\mathrm{A}_{4}>\mathrm{A}_{2}>\mathrm{A}_{1}$ \\
$(p=q=1)$ & $\mathrm{Q}_{1}=0.8984, \mathrm{Q}_{2}=0.9096$, & \\
\hline the proposed novel method & $\mathrm{Q}_{3}=0.9258, \mathrm{Q}_{4}=0.9109$. & \\
based on the IT2FWPBM operator & $(a=b=1)$ &
\end{tabular}

Note. $Q_{i}$ is the abbreviation of the ranking of $\mathrm{A}_{i}(i=1,2,3,4)$.

clarify these evidently different ranking orders based on the three methods.

Obviously, Zhang's method based on the GTIT2FWA operator and the GTIT2FHA operator [19] and the proposed novel method based on the IT2FWPHM operator $(p=1, q=$ 0 ) are not concerned with the interrelationships between attributes. As a rule, these rankings are not convincing because there are some specific interrelationships between attributes in this numerical example, i.e., between the service performance $\left(\mathrm{T}_{1}\right)$ and the supplier profile $\left(\mathrm{T}_{2}\right)$. Usually, the greater the service level, the greater the supplier profile. Therefore, we should account for this specific interrelationship between them.

Meanwhile, we also realize that the ranking order based on the proposed novel method ( $p=q=1)$ is the same as the one based on Wang et al.'s method [16] $(p=q=$ $1)$. It is clear that these two methods $(p=q=1)$ account for the interrelationships between attributes. It is well-documented that these rankings are more reasonable than Zhang's method based on the GTIT2FWA operator and GTIT2FHA operator [19] and the proposed novel method based on the IT2FWPHM operator $(p=1, q=0)$ in the practical problem.

Example 2. In some actual situations, the interrelationships between attributes only exist in the same partition, and there are no interrelationships between attributes in different partitions. In such a case, we modify the FELSP selection case by replacing $\widetilde{l}_{11}$ in Table 4 with the preference value EW. The other EVs remain unchanged. The ranking orders based on the different methods are listed in Table 10.

From Table 10, we discover that the ranking orders of Wang et al's method [16] based on the TIT2FWGHM operator $(p=q=1)$ are changed from $\mathrm{A}_{3}>\mathrm{A}_{4}>$ $A_{2}>A_{1}$ to $A_{3}>A_{2}>A_{4}>A_{1}$. Wang et al.'s method [22] based on the TIT2FWGHM operator accounts for the interrelationships between any two attributes. However, in case of FELSP selection, we divide those seven attributes into three partitions: $\mathrm{P}_{1}=\left\{\mathrm{T}_{1}, \mathrm{~T}_{5}\right\}, \mathrm{P}_{2}=\left\{\mathrm{T}_{2}, \mathrm{~T}_{3}, \mathrm{~T}_{4}\right\}$, and $\mathrm{P}_{3}=\left\{\mathrm{T}_{6}, \mathrm{~T}_{7}\right\}$. Furthermore, there are no interrelationships between $\mathrm{T}_{1}$ and $\mathrm{T}_{2}, \mathrm{~T}_{3}$, and $\mathrm{T}_{4}$. Similarly, there are no interrelationships between $\mathrm{T}_{1}$ and $\mathrm{T}_{6}$. Obviously, Wang et al's method [22] based on the TIT2FWGHM operator does not consider this case, and so its ranking is unreasonable. The proposed novel method based on the IT2FWPHM operator can satisfactorily deal with this problem since it perfectly removes the effects of the irrelevant attributes. Thus, the proposed novel method based on the IT2FWPHM operator is more rational than Wang e.al's method [22] based on the TIT2FWGHM operator.

In addition, compared with the existing two methods $[19,22]$, the proposed novel method also has the following favorable advantages, which are not contained in other literatures on methods based on the PHM operator.

(1) The novel proposed method determines the weights of attributes by applying MDM, which is an objective method. However, the two existing methods [19,22] apply a subjective method to determine the weights of attributes.

(2) In view of QFD, the EVs and weights of the CRs are converted into the EVs and weights of the TAs through the relationships between the CRs and TAs. In many practical contexts, it is unreasonable to make a direct assessment using the FELSP based on TAs. For instance, in most universities in China, if teachers plan to buy a portable computer (PC), the procurement division should select the best PC supplier on the basis of the teachers' requirements for PC performance. Directly selecting a PC supplier and passing over the teachers' requirements will probably lead to teachers' dissatisfaction. QFD can completely guarantee that the PC supplier's attributes are in accordance with the characteristics that the purchased PCs ought to possess. In this paper, the proposed novel method can guarantee customers' satisfaction and make the assessment more reasonable.

In the following, we compare the characteristics of the proposed method with the other two existing methods from $[19,22]$, which are listed in Table 11 . We can visually see that the proposed novel method does not suffer from the same limitations as the two existing methods in $[19,22]$.

\section{Conclusions}

IT2FSs are the most actively applied T2FSs which can more easily express vagueness and imprecision than T1FSs. QFD can give a competitive advantage to those who are able to pay close attention to customers' responses and needs in terms of LSPS. In this paper, the IT2FPHM operator and IT2FWPHM operator are developed and some of their properties are discussed. On this basis, a MCGDM method by combining QFD with PHM operator in the context of IT2FSs is proposed. The novel of this method is that the EVs and weights of CRs are converted into the EVs and weights of TAs with the relationships between CRs and TAs based on classical QFD. It can guarantee that LSP attributes 
TABLE 11: The comparison orders of the characteristics for different methods.

\begin{tabular}{lcccc}
\hline Methods & $\begin{array}{c}\text { Consider the } \\
\text { relationship between } \\
\text { attributes }\end{array}$ & $\begin{array}{c}\text { Consider the } \\
\text { partition of attributes }\end{array}$ & $\begin{array}{c}\text { Weights of attributes } \\
\text { based on objective } \\
\text { method }\end{array}$ & $\begin{array}{c}\text { The preference values } \\
\text { come from the } \\
\text { customer }\end{array}$ \\
\hline Zhang's method [19] & No & No & No & No \\
Wang et al.'s method [22] & Yes & No & No & No \\
the proposed method & Yes & Yes & Yes & Yes \\
\hline
\end{tabular}

are in accordance with the customer's satisfaction evaluation attributes and can ensure customers' satisfaction. At last, a case of FELSPS is utilized to illustrate the applicability of the presented method and some comparisons are used to display the superiority of the presented method.

For further research, some other aggregate operators, such as the partitioned Hamy mean operator, the partitioned Muirhead mean operator, and the partitioned Maclaurin symmetric mean operator, can be used to integrate IT2FSs in the LSPS problem. Meanwhile, we can also use the presented method to address other practical provider selection problems, such as sustainable supplier selection, green LSPS, and others.

\section{Data Availability}

The original evaluation data of customer and expert used to support the findings of this study are included within the supplementary information file(s) and the other data used to support the findings of this study are included within the article.

\section{Conflicts of Interest}

The authors declare that they have no conflicts of interest.

\section{Acknowledgments}

This paper is supported by the National Natural Science Foundation of China (nos. 71771140, 71471172, and 71801142), the Special Funds of Taishan Scholars Project of Shandong Province (no. ts201511045), and the Science Research Foundation of Heze University (no. XY16SK02).

\section{Supplementary Materials}

(1) ten customers are surveyed and interviewed with respect to the importance of the CRs by using provided LTs (see Tables 1 and 2); (2) four experts, involving third-party logistic service designer, logistic department manager, supply chain management professional, logistic management professor are summoned to give EVs of relationships between CRs and TAs by using provided LTs (see Tables 1 and 2). Due to the space constraints, we give only one customer's and one expert's EV in this paper. The data about nine customers and three experts are included in supplementary file. (Supplementary Materials)

\section{References}

[1] K. Govindan, M. Kadziński, R. Ehling, and G. Miebs, "Selection of a sustainable third-party reverse logistics provider based on the robustness analysis of an outranking graph kernel conducted with ELECTRE I and SMAA," OMEGA - The International Journal of Management Science, 2018.

[2] M. G. Speranza, “Trends in transportation and logistics," European Journal of Operational Research, vol. 264, no. 3, pp. 830836, 2018.

[3] W. Liu, X. Shen, and D. Xie, "Decision method for the optimal number of logistics service providers with service quality guarantee and revenue fairness," Applied Mathematical Modelling: Simulation and Computation for Engineering and Environmental Systems, vol. 48, pp. 53-69, 2017.

[4] X. Hu, G. Wang, X. Li, Y. Zhang, S. Feng, and A. Yang, "Joint decision model of supplier selection and order allocation for the mass customization of logistics services," Transportation Research Part E: Logistics and Transportation Review, vol. 120, pp. 76-95, 2018.

[5] A. Martikainen, P. Niemi, and P. Pekkanen, "Developing a service offering for a logistical service provider-case of local food supply chain," International Journal of Production Economics, vol. 157, no. 1, pp. 318-326, 2014.

[6] W. Chen, M. Goh, and Y. Zou, "Logistics provider selection for omni-channel environment with fuzzy axiomatic design and extended regret theory," Applied Soft Computing, vol. 71, pp. 353-363, 2018.

[7] M. Yazdani, P. Chatterjee, E. K. Zavadskas, and S. Hashemkhani Zolfani, "Integrated QFD-MCDM framework for green supplier selection," Journal of Cleaner Production, vol. 142, no. 2, pp. 3728-3740, 2017.

[8] Y.-L. Li, C.-S. Ying, K.-S. Chin, H.-T. Yang, and J. Xu, “Thirdparty reverse logistics provider selection approach based on hybrid-information MCDM and cumulative prospect theory," Journal of Cleaner Production, vol. 195, pp. 573-584, 2018.

[9] D. Pamucar, K. Chatterjee, and E. Zavadskas, "Assessment of third-party logistics provider using multi-criteria decisionmaking approach based on interval rough numbers," Computers \& Industrial Engineering, vol. 127, pp. 383-407, 2019.

[10] M. N. Shaik and W. Abdul-Kader, "A hybrid multiple criteria decision making approach for measuring comprehensive performance of reverse logistics enterprises," Computers \& Industrial Engineering, vol. 123, pp. 9-25, 2018.

[11] L. A. Zadeh, "The concept of a linguistic variable and its application to approximate reasoning I," Information Sciences, vol. 8, pp. 199-249, 1975.

[12] J. M. Mendel, R. I. John, and F. Liu, "Interval type-2 fuzzy logic systems made simple," IEEE Transactions on Fuzzy Systems, vol. 14, no. 6, pp. 808-821, 2006. 
[13] H. Li, J. Wang, L. Wu, H. K. Lam, and Y. Gao, "Optimal guaranteed cost sliding mode control of interval type-2 fuzzy time-delay systems," IEEE Transactions on Fuzzy Systems, 2017.

[14] I. Eyoh, R. John, G. De Maere, and E. Kayacan, "Hybrid learning for interval type-2 intuitionistic fuzzy logic systems as applied to identification and prediction problems," IEEE Transactions on Fuzzy Systems, vol. 26, no. 5, pp. 2672-2685, 2018.

[15] M. K. Ghorabaee, M. Amiri, E. K. Zavadskas, Z. Turskis, and J. Antucheviciene, "A new multi-criteria model based on interval type-2 fuzzy sets and EDAS method for supplier evaluation and order allocation with environmental considerations," Computers \& Industrial Engineering, vol. 112, pp. 156-174, 2017.

[16] D. S. Comas, J. I. Pastore, A. Bouchet, V. L. Ballarin, and G. J. Meschino, "Interpretable interval type-2 fuzzy predicates for data clustering: A new automatic generation method based on self-organizing maps," Knowledge-Based Systems, vol. 133, pp. 234-254, 2017.

[17] P. Kundu, S. Kar, and M. Maiti, "A fuzzy multi-attributes group decision-making based on ranking interval type-2 fuzzy variables and an application to transportation mode selection problem," Soft Computing, vol. 21, no. 11, pp. 3051-3062, 2017.

[18] Y. Gong, N. Hu, J. Zhang, G. Liu, and J. Deng, "Multi-attribute group decision making method based on geometric Bonferroni mean operator of trapezoidal interval type-2 fuzzy numbers," Computers \& Industrial Engineering, vol. 81, pp. 167-176, 2015.

[19] Z. Zhang, "Trapezoidal interval type-2 fuzzy aggregation operators and their application to multiple attribute group decision making," Neural Computing and Applications, vol. 29, no. 4, pp. 1039-1054, 2018.

[20] G. Beliakov, A. Pradera, and T. Calvo, Aggregation Functions: A Guide for Practitioners, Springer, Berlin, Germany, 2007.

[21] P. Liu and S.-M. Chen, "Group decision making based on heronian aggregation operators of intuitionistic fuzzy numbers," IEEE Transactions on Cybernetics, 2016.

[22] H. Wang, Y. Ju, P. Liu et al., "Some trapezoidal interval type2 fuzzy Heronian mean operators and their application in multiple attribute group decision making," Journal of Intelligent \& Fuzzy Systems, 2018.

[23] P. Liu, S.-M. Chen, and J. Liu, "Multiple attribute group decision making based on intuitionistic fuzzy interaction partitioned Bonferroni mean operators," Information Sciences, vol. 411, pp. 98-121, 2017.

[24] Z. Liu, P. Liu, W. Liu, and J. Pang, "Pythagorean uncertain linguistic partitioned Bonferroni mean operators and their application in multi-attribute decision making," Journal of Intelligent \& Fuzzy Systems: Applications in Engineering and Technology, vol. 32, no. 3, pp. 2779-2790, 2017.

[25] Y. Wang and P. Liu, "Linguistic neutrosophic generalized partitioned Bonferroni mean operators and their application to multi-attribute group decision making," Symmetry, vol. 10, no. 5, 2018.

[26] K. Yin, B. Yang, and X. Li, "Multiple attribute group decisionmaking methods based on trapezoidal fuzzy two-dimensional linguistic partitioned bonferroni mean aggregation operators," International Journal of Environmental Research and Public Health, vol. 15, no. 2, 2018.

[27] P. Liu, J. Liu, and J. M. Merigó, "Partitioned Heronian means based on linguistic intuitionistic fuzzy numbers for dealing with multi-attribute group decision making," Applied Soft Computing, vol. 62, pp. 395-422, 2018.
[28] M. M. Chowdhury and M. A. Quaddus, "AA multiple objective optimization based QFD approach for efficient resilient strategies to mitigate supply chain vulnerabilities: The case of garment industry of Bangladesh," Omega, vol. 57, pp. 5-21, 2015.

[29] C.-N. Liao and H.-P. Kao, "An evaluation approach to logistics service using fuzzy theory, quality function development and goal programming," Computers \& Industrial Engineering, vol. 68, no. 1, pp. 54-64, 2014.

[30] M. Yazdani, C. Kahraman, P. Zarate, and S. C. Onar, "A fuzzy multi attribute decision framework with integration of QFD and grey relational analysis," Expert Systems with Applications, vol. 115, pp. 474-485, 2019.

[31] Y. Li, D. Kannan, K. Garg, S. Gupta, K. Gandhi, and P. C. Jha, "Business orientation policy and process analysis evaluation for establishing third party providers of reverse logistics services," Journal of Cleaner Production, vol. 182, pp. 1033-1047, 2018.

[32] M. Li, L. Jin, and J. Wang, "A new MCDM method combining QFD with TOPSIS for knowledge management system selection from the user's perspective in intuitionistic fuzzy environment," Applied Soft Computing, vol. 21, pp. 28-37, 2014.

[33] C. P. M. Govers, "What and how about quality function deployment (QFD)," International Journal of Production Economics, vol. 46-47, pp. 575-585, 1996.

[34] J. M. Mendel, "Type-2 fuzzy sets and systems: an overview," IEEE Computational Intelligence Magazine, vol. 2, no. 1, pp. 2029, 2007.

[35] J. Qin, X. Liu, and W. Pedrycz, "An extended TODIM multicriteria group decision making method for green supplier selection in interval type-2 fuzzy environment," European Journal of Operational Research, vol. 258, no. 2, pp. 626-638, 2017.

[36] M. Keshavarz Ghorabaee, E. K. Zavadskas, M. Amiri, and A. Esmaeili, "Multi-criteria evaluation of green suppliers using an extended WASPAS method with interval type-2 fuzzy sets," Journal of Cleaner Production, vol. 137, pp. 213-229, 2016.

[37] J. D. Qin, X. W. Liu, and W. Pedrycz, "An extended VIKOR method based on prospect theory for multiple attribute decision making under interval type-2 fuzzy environment," KnowledgeBased Systems, vol. 86, pp. 116-130, 2015.

[38] J. A. Carnevalli and P. C. Miguel, "Review, analysis and classification of the literature on QFD-Types of research, difficulties and benefits," International Journal of Production Economics, vol. 114, no. 2, pp. 737-754, 2008.

[39] Y. M. Wang, "Using the method of maximizing deviations to make decision for multi-indices," Journal of Systems Engineering and Electronics, vol. 7, pp. 24-26, 1998. 


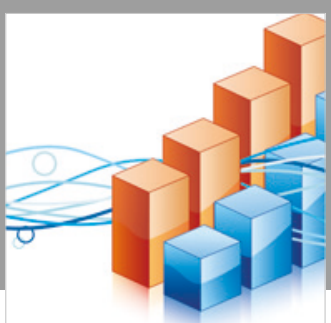

Advances in

Operations Research

\section{-n-m}
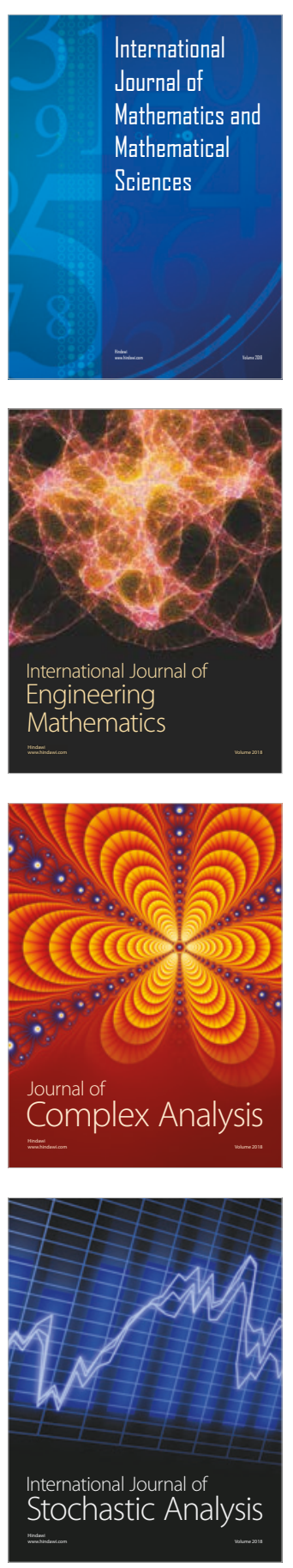
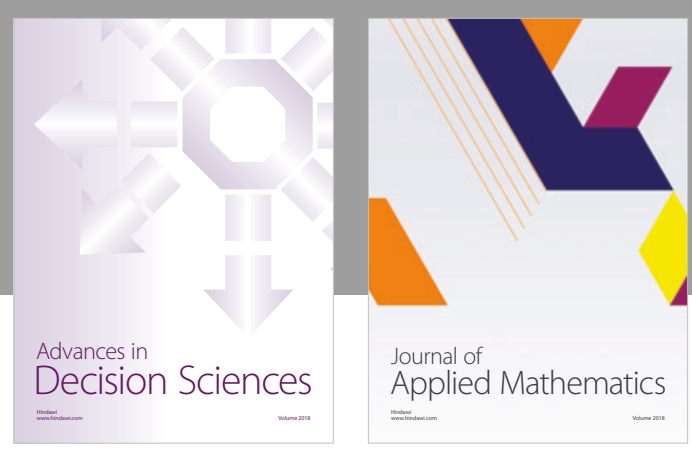

Journal of

Applied Mathematics
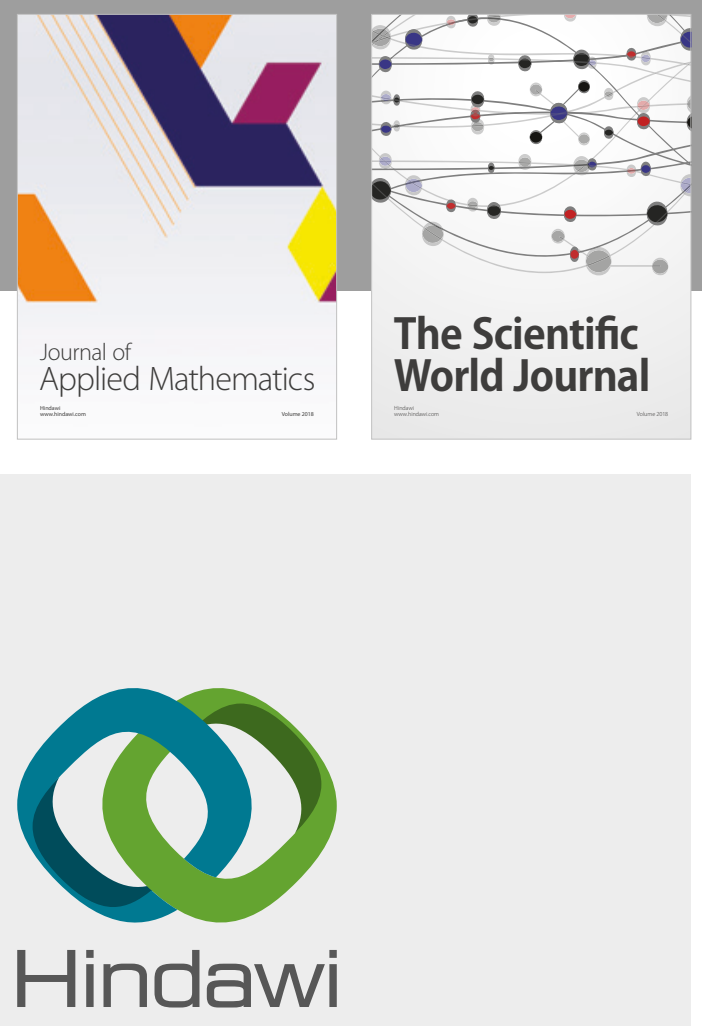

Submit your manuscripts at

www.hindawi.com

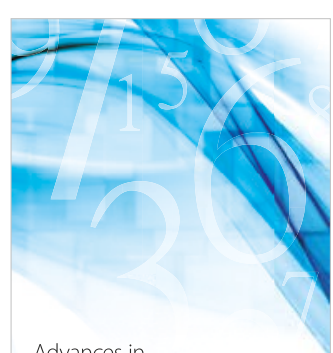

Advances in
Numerical Analysis
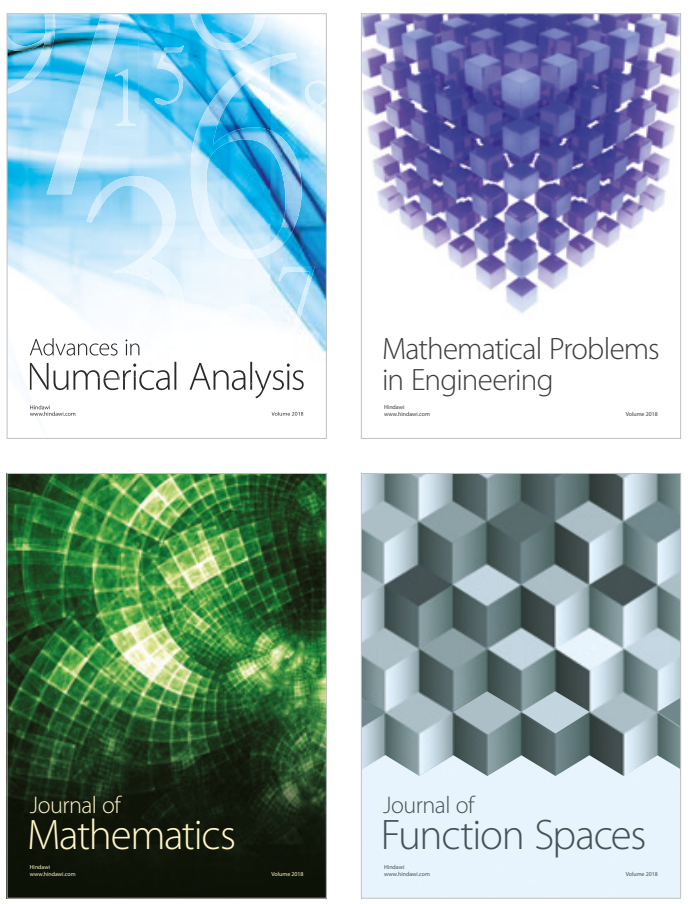

Mathematical Problems in Engineering

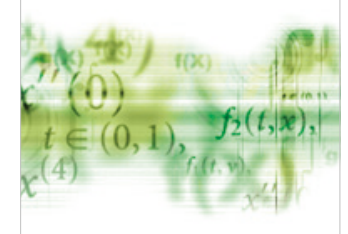

International Journal of

Differential Equations

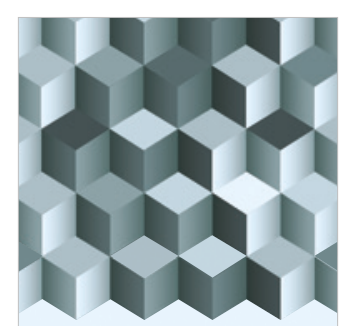

Journal of

Function Spaces
The Scientific

World Journal

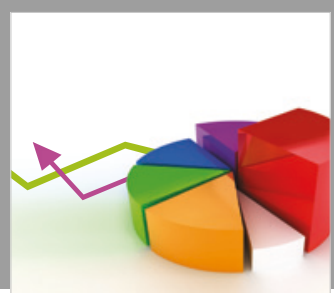

Journal of

Probability and Statistics
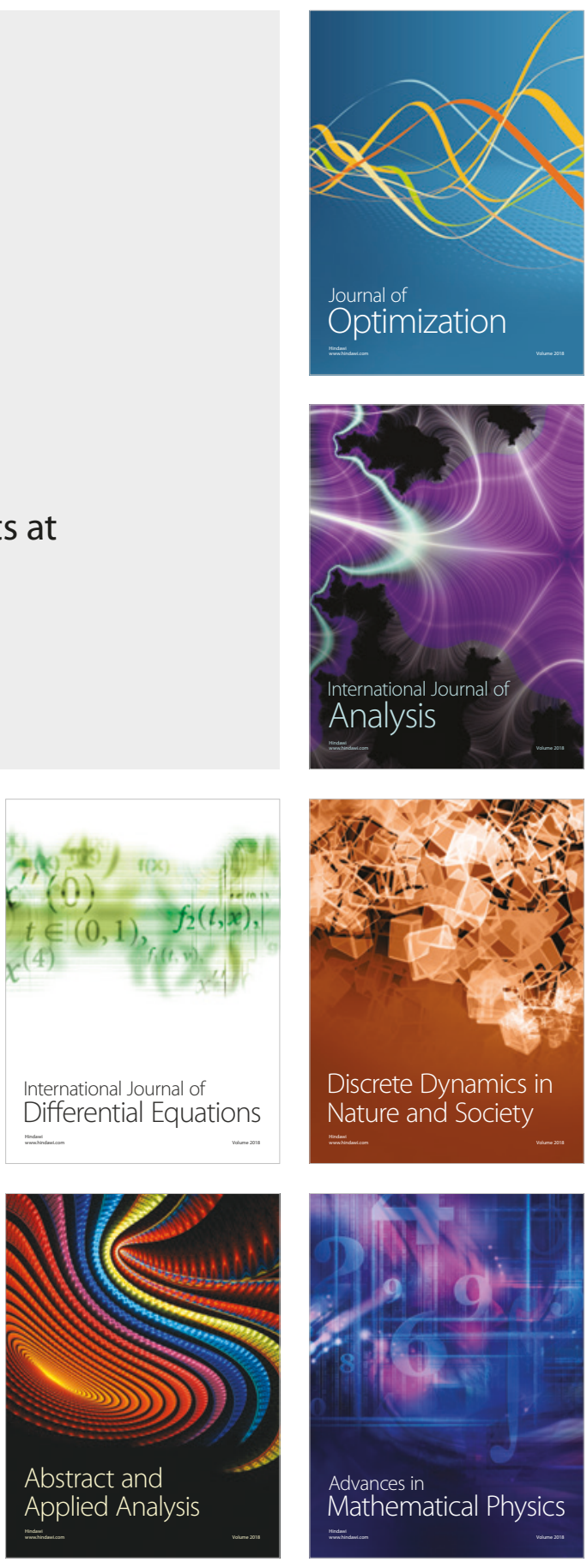